

\section{Risk-Aware Design of Value and Coordination Networks}

Hassan Fatemi

Enschede, the Netherlands, 2012

CTIT Ph.D.-Thesis Series, No. 12-232 
Ph.D. dissertation commitee:

Chairman and secretary:

Promotor:

Assistant Promotors:

Members:
Prof.dr.ir. A.J. Mouthaan

(University of Twente, the Netherlands)

Prof.dr. R. J. Wieringa

(University of Twente, the Netherlands)

Dr.ir. M. J. van Sinderen

(University of Twente, the Netherlands)

Dr. J. Gordijn

(Vrije Universiteit Amsterdam, the Netherlands)

Prof.dr. H. Afsarmanesh

(University of Amsterdam, the Netherlands)

Prof.dr. P. Johnson

(KTH - Royal Institute of Technology, Sweden)

Prof.dr.ir L.J.M. Nieuwenhuis

(University of Twente, the Netherlands)

Dr. L. Ferreira Pires

(University of Twente, the Netherlands)

CTIT Ph.D.-Thesis Series, No. 12-232

CT1 Centre for Telematics and Information Technology P.O. Box 217, 7500 AE Enschede, The Netherlands

SIKS Dissertation Series, No. 2012-39

sTres

The research reported in this thesis has been carried out under the auspices of SIKS, the Dutch Research School for Information and Knowledge Systems.

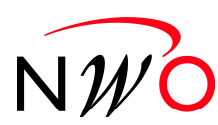

This research has been financially supported by Nederlandse organisatie voor Wetenschappelijke Onderzoek (NWO) under contract No. 638.001 .205

Printed and bound by Ipskamp Drukkers B.V., The Netherlands

Cover design by Ashkan Golshahi and Hajir Gharib

ISSN $1381-3617$

ISBN 978-90-365-3437-6

http://dx.doi.org/10.3990/1.9789036534376

Copyright (C) 2012, Hassan Fatemi, the Netherlands

All rights reserved. Subject to exceptions provided for by law, no part of this publication may be reproduced, stored in a retrieval system, or transmitted, in any form or by any means, electronic, mechanical, photocopying, recording or otherwise, without the prior written permission of the copyright owner. No part of this publication may be adapted in whole or in part without the prior written permission of the author. 


\title{
RISK-AWARE DESIGN OF VALUE AND COORDINATION NETWORKS
}

\author{
DISSERTATION
}

to obtain

the degree of doctor at the University of Twente, on the authority of the rector magnificus,

Prof. dr. H. Brinksma,

on account of the decision of the graduation committee, to be publicly defended

on Wednesday, 10th of October 2012 at 16.45

by

\section{Hassan Fatemi}

born on 21 January 1982

in Baneh, Kurdistan, Iran 
Dit proefschrift is goedgekeurd door:

Prof.dr. R. J. Wieringa (promotor) en Dr.ir. M. J. van Sinderen (assistentpromotor)

Design Your Future. 


\section{Abstract}

A collaborative network is a network consisting of a set of autonomous actors (e.g. enterprises, organizations and people) that collaborate to achieve common or compatible goals. In a collaborative network each enterprise contributes with its own specific products or services to satisfy the consumer need. Actual collaborative networks are often risky ventures because there is no central coordinator. A collaborative network that emerges gradually is not subject to the same risks as the participants engage in the network slowly. We develop techniques for designing IT-enabled collaborative networks and validating the risk that they entail. Some of the risks threatening a collaborative network designed in advance are:

- the risk of non-executability (no feasible coordination)

- the risk of fraud or in general untrustworthy partners

- the risk of non-profitability

- the risk of loss of business-confidential data

In this work we address the first three types of risks.

Designing collaborative networks calls for modeling the collaboration of enterprises from different perspectives, in particular the value and coordination perspectives, and for mutually aligning these perspectives. The need for these two perspectives stems from the importance of separating the how from the what concerns. The value perspective focuses on what is offered by whom to whom while the coordination perspective focuses on how these offerings are fulfilled operationally. Value modeling and coordination modeling have different goals and use different concepts. Nevertheless, the resulting models should be consistent with each other because they refer to the same system.

The model that shows the creation, distribution, and consumption of goods or services of economic value in a collaborative network is called value model. The main goal of value modeling is to build a shared understanding of the business case and reach agreement amongst profit-and-loss responsible business actors re- 
garding the question "Who is offering what of value to whom and expects what of value in return?" It also enables the actors to assess their potential profitability in the collaborative network to develop an insight into the economic viability and sustainability of the whole collaborative network. The study of the relation between value and coordination models is to address the executability of the collaborative network.

The participating actors in a collaborative network are assumed to act trustfully in the collaboration and therefore trust is mostly left entirely outside the picture. However the assumption that business actors act trustfully is often not useful in practice (since there are malicious actors). Each partner agrees to act according to the value model, however during the business, actors need to be sure if their partners are acting according to the value model or not. Dropping the trust assumptions in business collaboration is to address the risk of untrustworthy partners. Finally, after taking the trust complications into account, we need to adjust the profitability and sustainability according to the new setting and hence address the risk of reduced profitability or even loss in the collaboration.

The contributions of this thesis include:

1. Introducing a stepwise method to produce a consistent coordination model from a value model and vice versa in a multiperspective e-collaborative network design.

2. Analyzing collaborative networks from a trust perspective and introducing an approach to measure the trustworthiness of the actors participating in a collaborative network.

3. Assessing profitability and sustainability of some special collaboration settings using game theory concepts and techniques and refining the initial profitability analysis of the collaborative network by taking trust into account.

We evaluate our findings using some real business collaboration cases. 


\section{Acknowledgements}

Four years ago, I made the challenging decision to pursue my academic career abroad. Leaving all your friends, family and country - a place you had lived 26 years - to go alone to another country that from many perspectives is another world makes for a real challenge. One has to build many things from scratch. Despite being away from all loved ones, living here has been quite an adventure: a fantastic job, visiting amazing places (US, Brazil, Japan and different countries in Europe) which I had only visited in dreams before my $\mathrm{PhD}$, and above all, working in an incomparable office with a group of people I would like to thank.

First and the foremost, I would like to thank my promotor Roel Wieringa and my supervisor Marten van Sinderen. Thank you Roel for believing in me from the very beginning by accepting me without an interview and for giving me enough freedom to pursue my own research interests. Your high level and helicopter view helped me a lot and your ever-positive attitude made me think of you as a friend rather than a boss! Thank you Marten for being a constant source of motivation and inspiration. Your detailed discussions, comments and feedback influenced this thesis greatly. Roel and Marten, you are a perfect combination of supervision that a $\mathrm{PhD}$ candidate could hope for.

I would like to thank the members of my defense committee and in particular Luis Ferreira Pires. Thank you Luis for your meticulous comments and feedbacks; They polished this dissertation.

I could not imagine the IS group without Suse, as one cannot imagine a family without a mother. Dear Suse, you deserve at least half of my $\mathrm{PhD}$ title and I wish I could share it with you. Thank you for continuously helping me and reminding me of all my schedules and appointments! One month ago, I came to you and told you that I should have filled-in a form regarding my PhD defense ceremony but I did not and now I cannot find it and I do not even remember what was it exactly about! Like 
always, you figured out the dilemma! colleagues from IS group: Silja, Lianne, Mohammad, Shahin, Andre, Brahmananda, Camlon, Wilco, Eelco, Steven, Wolter, Jelena, Nelly, Chen, Roberto. Thank you all for making IS group a pleasant working place.

Silja, my encyclopedia, thank you for all your support. Mohammad you are a true friend. I really enjoyed all those times which we spent together specially our workouts at the gym. Thank you for constantly updating and helping me with all those things which I should have taken care of as an Iranian living in the Netherlands. You and Suse made a magical combination of support. Brahmananda I appreciate our brief talks during short breaks.

Mehrdad, Babak, Saeed, Shervin we shared a lot of laughter and fun moment together. Mehrdad and Babak I learned a lot from you and I thank you for that. Ashkan and Hajir thank you for designing the cover and saving me at the last minute.

The special thank goes to my family and friends back home and specially to my beloved parents. Even though most of you are not able to read this thesis, not even this paragraph, but I still would like to thank you for all your everlasting support throughout my life, for your true love, for believing in me and making me feel that I can achieve anything. 


\section{Contents}

Chapter 1: Introduction 1

1.1 Collaborative Networks 2

1.2 Problems and Challenges 5

1.3 Research Problems 8

1.4 Research Methodology 9

1.5 Thesis Structure 10

Chapter 2 : Basic Concepts and Notations 13

2.1 Value Modeling 14

2.2 Coordination Modeling 19

2.3 Game Theory 21

Chapter 3 : From Value Model to Coordination Model 27

3.1 Value Model vs Coordination Model 28

3.2 From Value Model to Coordination Model 33

3.3 Case Study 41

3.4 Discussion and Conclusions 46

Chapter 4: From Coordination Model to Value Model 49

4.1 From Coordination Model to Value Model 50

4.2 Discussion and Conclusions 54

Chapter 5: Automation of Model Transformation 57

5.1 Model Transformation $\quad 58$

5.2 Automation of Transformation 60

5.3 Discussion and Conclusions 65

Chapter 6: $\quad$ Trust and Collaborative Networks 67

6.1 Introduction 68

6.2 Trust 70

6.3 Trust Ontology for Business collaboration Settings $\quad 73$

6.4 Managing Trust within Collaborative Networks $\quad 77$

6.5 Discussion 87

6.6 Related Work $\quad 89$

6.7 Conclusions 90 
Chapter 7 : Managing Trust in Collaborative Networks using Game Theory

7.1 Introduction 94

7.2 Observable vs. Unobservable Value Exchanges 96

7.3 Inspection Game 97

7.4 Discussion 103

$\begin{array}{lll}7.5 & \text { Conclusions } & 105\end{array}$

Chapter 8: Endurability and Profitability Analysis of Collaborative Networks 107

8.1 Endurability of Collaborative Networks 108

8.2 Profitability Analysis of Collaborative Networks 113

8.3 An Illustrative Case Study 117

8.4 Conclusions 121

$\begin{array}{lll}\text { Chapter 9 : } & \text { Validation } & 123\end{array}$

9.1 Introduction 123

9.2 Renewable Electricity in the UK 125

9.3 Excise collection within the EU 132

9.4 Creating Value for All: Community-Based Tourism 138

$\begin{array}{lll}\text { Chapter } 10 & \text { Conclusions } & 147\end{array}$

10.1 Contributions 147

10.2 Future Research 151

$\begin{array}{ll}\text { References } & 155\end{array}$

$\begin{array}{lr}\text { Author Publications } & 167\end{array}$

$\begin{array}{lr}\text { SIKS Dissertation Series } & 169\end{array}$

$\begin{array}{lr}\text { Samenvatting } & 187\end{array}$ 


\section{Introduction}

"A scientist can discover a new star, but he cannot make one. He would have to ask an engineer to do that."

- Gordon L. Glegg, American Engineer, 1969.

The traditional thinking of value creation in industrial economy considers companies as actor in a value chain. Each company in this chain receives some input from upstream companies, adds value to this input, and then passes the result downstream to the next actor in the chain (another company or the final customer). This understanding of value creation is out-dated. Global competition and the dynamicity of the market and the new technologies are defining new ways of creating value. In the new competitive environment, successful companies do not just add value, they reinvent it, i.e. they focus on a value creating system, within which different business actors (e.g. suppliers, enterprises, business partners, and customers) work together to co-produce value [1].

Developments in Information Technology (IT) has changed many facets of everyday life. The way people collaborate in a business is no exception. IT advancements and in particular the widespread use of Internet provide many opportunities for businesses. Some of these opportunities are not obvious and they need an innovative idea to be pursued and operationalized. At the same time, technology advancements have made it impossible for one enterprise to do everything on its own, i.e. the new products ask for collaboration between different enterprises with different expertises and competencies. Consequently, nowadays business collaboration between different enterprises and actors in an IT enabled context has become a prevailing trend.

Today, enterprises operate more and more together in networked collaborations rather than just on their own. Collabo- 
ration of different enterprises not only generates a synergy that can contribute to the competitive advantage of all participating enterprises but it also reduces the risk of failure by sharing the loss. This is of particular importance because new product development is inherently risky, especially when new technology or emerging markets are involved [2]. Nevertheless such collaboration has its own limitations. It can also bring many of the latent risks in the product development process to the surface, specifically if the collaboration spans geographical as well as organizational boundaries [1].

Business collaborative networks are nothing new however the important change is that nowadays their creation and operation is made possible by IT. IT is not only a way to automate existing business but also a way to create new business. The same story holds for risks: Doing business is engaging in risk, and this has always been the case. But due to the size and dynamics of the networks that can be created using IT, the risks may be higher, but the means to assess and manage them have also increased.

Attesting the profitability and feasibility of the collaboration for all participating actors is the first step in every business collaboration. Those who decide to join a business collaboration are mostly business men who lack knowledge about technical detail of the operation of the collaboration. Therefore we need to provide non technical business decision makers with models that are precise and accurate enough and at the same time easy enough to understand. Such initial business models are the basis for models that address the technical details of the operation of the collaboration. The study of the relation between different models of a business collaboration and the mutual consistency between these models constitute part of the work in this thesis.

More specifically, this thesis tries to bridge the gap between business models and technical models of IT, and also provides ways to assess risk in such IT-enabled business collaborative networks.

\subsection{Collaborative Networks}

Nowadays, the networks that enterprises operate in become increasingly complex. There are many reasons for this. Among others we can refer to more advanced user needs, upward tendency toward specialization, changing customer demands, higher customer satisfaction criteria, advancement in information and 
communication technology (ICT), globalization of markets and manufacturing, increasing competitiveness, exposure to a bigger audience, etc. In fact, collaboration of different enterprises to co-produce a product or service is nothing new, however, here in this thesis, we focus only on those business collaborations which are facilitated by ICT. In other words, we are concerned with the design and use of IT in IT-enabled business collaborations.

A collaborative network is a network consisting of a set of autonomous actors (e.g. enterprises, organizations and people) that collaborate to achieve common or compatible goals [3, 4]. Collaborative networks come with different names in the literature, such as business webs [5], Virtual enterprises (VE) [6, 7], extended enterprises [8, 9], strategic alliances [10, 11], value constellations $[12,13,14]$.

Tapscott et al. define a business web as a collection of enterprises designed to jointly satisfy a consumer need [5]. Katzy defines virtual enterprise as a temporary co-operation to realize the value of a short-term business opportunity that none of the partners can capture on its own [6]. The virtual enterprise has a dynamic environment where individual enterprises work together for a relatively short time, to satisfy niche market demands quickly whereas the concept of the extended enterprise focuses on long-term enterprise relationships across the value chain. The extended enterprise is responsible for the whole product life cycle and it includes the relationships that an enterprise has with its customers, suppliers, business partners, even former competitors and so on [8]. A strategic alliance is a cooperation or collaboration that aims for a synergy where each partner hopes that the benefits from the alliance will be greater than those from individual efforts. The alliance often involves technology transfer (access to knowledge and expertise), economic specialization, shared expenses and shared risk [11]. Normann and Ramirez [12, 13] introduce the value constellation as a successor of the value chain [15]. A value constellation is a construct where actors come together to co-produce value with each other. The aim of the value constellation theory is to help actors with continuously (re)designing their business.

The collaboration of enterprises to co-produce a product/service is the common theme of all the above definitions. Nevertheless, this collaboration can have different forms based on the degree of commitment and the level of engagement of the enterprises involved. For example, those enterprises that provide complementary products or services in a touristic area can make 
a collaborative relationship in which many local tourism providers collaborate in order to market a particular location as a fun tourism destination. This would involve hospitality service providers like hotels, B\&Bs, guesthouses, and self catering owners working closely with providers of activity-based interests (horse riding, water sports, biking, hiking, historical places of interest, fishing, quad biking, etc.) to create a compelling holiday destination touristic value proposition for a specific area. This type of collaboration can be created by publishing joint brochures, recommending each others' services to existing and potential customers, creating a hyperlink on each others' websites, etc.

Due to the complementary nature of their products and services, these companies would be more successful and have more profitability if they collaborate. At the same time, they can still do their own business outside the collaboration and if one partner fails in delivering the promised service/product it does not necessarily terminate the collaboration. In fact this type of collaboration is not obligatory and therefore it does not dictate a specific action on the participants.

In contrast to the non-obligatory business collaborations, there are business collaborations in which each partner contributes with its own specific products or services to achieve a common goal that cannot be achieved when one of the actors drops out of the collaboration. Therefore the collaboration of all partners is necessary to achieve the common goal of the collaboration. If one of the partners fails in delivering his/her undertakings and responsibilities then no other partner can achieve its goal(s) and consequently the collaboration fails. For example, a web shop, a logistics company, a payment provider and an authentication provider can jointly provide the on-line shopping service to consumers. In this collaboration, if for example the logistic company fails in delivering its undertakings, the collaboration fails. Other examples of these collaborative networks with non-optional participation, including an example from the tourism industry are provided in chapter 9 . This type of collaboration manifests a joint work and here in this thesis we address this kind of collaboration.

Nayak et al. [7] enumerate different layers for a solution architecture in a business collaboration namely, the technology layer, the Business to Business (B2B) integration layer, the e-marketplace service layer, the e-commerce solution layer, the dynamic e-business layer, and finally the B2B collaboration layer. Considering these layers, the topics covered in this thesis fall in the B2B integration layer, the e-business layer and the B2B collaboration layer. 
We use business value models to model collaborative networks. A business value model helps the collaboration partners share their understanding regarding the collaboration and enables them to analyze the economic profitability of the collaborative network. The main goal of business value modeling is to reach agreement amongst profit-and-loss responsible actors regarding the question "Who is offering what of value to whom and expects what of value in return?" We use the $e^{3}$ value method to describe/represent collaborative networks from the value point of view [14]. $E^{3}$ value is supported by a tool ${ }^{1}$ that allows profitability analysis for all partners in the network.

In the current state of the art, the collaboration partners are assumed to be trustworthy while designing value models. However, in real life, not all partners are equally trustworthy and, therefore, before a business value model can be put into operation, any unrealistic trust assumptions that it contains, must be removed.

\subsection{Problems and Challenges}

The work in this thesis addresses three main challenges/risks in business collaborations, namely 1) risk of non-executabile/nonfeasible inter-organizational processes (coordination), 2) risk of untrustworthy partners, and 3) risk of non-profitability. Below we elaborate on these challenges.

\subsubsection{Non-executability/non-feasibility risk}

A collaborative network is a multi perspective phenomenon and to study the challenges and issues related to a collaborative network we need to model it from different perspectives, in particular the value and coordination perspectives, and for mutually aligning these perspectives. One of the challenges of studying collaborative networks is investigating the relations between these two models and possibly generating one based on the other so that the resulting models are consistent with each other.

The main goal of value modeling is to reach agreement amongst profit-and-loss responsible actors regarding the question "Who is offering what of value to whom and expects what of value in return?" In contrast, an important goal of coordination modeling

\begin{tabular}{lll}
\hline$e^{3}$ value tool can be downloaded & at \\
http://e3value.few.vu.nl/tools/index.php
\end{tabular}


is to reach a common understanding about which coordination activities should be carried out, by whom and in which order. These are two different modeling goals, asking for different modeling methods with different constructs [16]. Nevertheless, despite the differences, a value model and its corresponding coordination model should be consistent with each other because they both refer to the same system. For modeling collaborative networks from value perspective, we use value models of $e^{3}$ value [14], and for the coordination perspective, we use BPMN diagrams [17]. However the generality of our results is beyond these two particular notations.

The issue of modeling a business collaboration from value and coordination perspective and studying the relation between these two models address the risk of executability/feasability of the business collaboration. Previous works $[18,19,20,21,22,23,24,25$, 26 in this area do not establish a firm relation between these two kinds of models and most of them [18, 20, 21, 26] study the issue only from a specific aspect and in a specific context. Here we investigate the relation between these two types of models in more detail and in a broader context so that the findings and guidelines are more general and applicable in different contexts. This issue is discussed in detail in Chapters 3, 4 and 5 .

\subsubsection{Risk of untrustworthy partners}

The value model assumes that all partners in the collaborative network behave in accordance with the rules and promises applicable to the network, because otherwise the collaboration does not serve the purpose of all actors. However, the risk in any business network is that a partner will not behave according to the value model and act in favor of its own goals, to the detriment of other partners' goals. We call this opportunistic behaviour.

In fact, for doing any business in the real world, trust is crucial for the success of the business. This forces a business to take appropriate and sufficient measures against those who it does not trust, i.e. who may not live up to their commitments. Therefore we need to model trust explicitly as a key component of the collaboration between parties who will benefit from the collaboration, but who must also worry about possible opportunistic behaviors of some of the partners.

The trust issue has not received enough attention in previous works [27, 28, 29] and they mainly introduce some guidelines for business modelers and precautions for business decision makers to take into account rather than providing them with an applicable 
method or tool. We want to provide the collaborative network designer with a tool to assess the trustworthiness of the actors participating in the collaborative network, and to use this assessment to redesign the value model and/or design the coordination and IT architecture in such a way that the designer can judge whether the remaining trust assumptions take an economically acceptable risk. We discuss the issue of untrustworthy partners in detail in Chapters 6 and 7.

\subsubsection{Risk of non-profitability}

The next issue we address, is profitability and sustainability assessments of collaborative networks. In addition to helping the actors develop a common understanding about the business collaboration, value modeling also enables the actors to assess their potential profitability in the collaborative network and therefore develop an insight into the economical viability and sustainability of the whole collaborative network. Each partner wants to be sure that participation in the collaborative network is economically rational and sustainable before specifying the coordination for the participation.

An $e^{3}$ value model consists of a graphic part and a computational part [27]. The graphic part is a diagram and the computational part is a spreadsheet with algorithms that can perform Net Present Value (NPV) estimations for the participating actors in the diagram. However, as mentioned before, value models represented in $e^{3}$ value methodology depict an ideal situation in which all actors are supposed to be trusted (i.e. all actors are assumed to act according the agreements made in the value model). This is not a realistic assumption and to provide the actors with a better and more accurate profitability and sustainability estimation of the collaborative networks, we need to drop this assumption and take the risks associated with opportunistic behaviors of the actors into account.

Currently the $e^{3}$ value methodology does not take trust into account for profitability analysis in collaborative networks and therefore we tackle this shortcoming. Using the established concepts and techniques of game theory $[30,31,32]$ we provide the actors with a better and more accurate view regarding the profitability and sustainability of collaborative networks. The profitability issue of business collaborations is addressed in Chapter 8 . 


\subsection{Research Problems}

The research in this thesis contributes to the inter-organizational business collaboration area and based on the challenges/risks identified in Section 1.2, we enumerate the following research problems:

- P1: The first research problem consists of three sub-problems which are:

1. P1.1: How to generate a coordination model based on a value model?

2. P1.2: How to generate a value model based on a coordination model?

3. P1.3: How to define and check the consistency between a value model and a coordination model?

The context of this research problem is the design of e-business networks and the stakeholder of this research problem is the designer of the e-business networks. The main requirement of the solution of the first two sub-questions (P1.1 and P1.2) is that the generated model should be consistent with the base (value or coordination) model and regarding the first subquestion, we should be able to feed the coordination model generated from the value model into some transformation or execution tool to execute it.

- P2: How to model the trustworthiness of business actors involved in a business collaboration so that actors can develop a better understanding regarding the trustworthiness of their partners in the collaboration and make a more stable and durable collaboration?

The context of this research problem is also the design of ebusiness networks, and the stakeholders of this research problem can be both the designer of the e-business networks and also the business actors. The main requirement for the solution of this research problem is that the proposed solution should be able to help the business actor assess the credibility of their trust assumptions. The model helps the business actors check how realistic their trust assumptions are.

- P3: This research problem consists of two sub-problems which are:

1. P3.1: How to analyze the profitability of an unobservable value exchange (a value exchange that needs inspection)?

2. P3.2: How to assess the profitability and sustainability of the collaborative networks by taking trust into account? 
The context of this research problem is also the design of ebusiness networks and the stakeholders of this research problem can be both the designer of the e-business networks and also the business actors. In case of the first sub-problem, the result of the analysis should be consistent with the financial expectations of the business actors and regarding the second sub-problem, we should be able to use the proposed approach in conjunction with $e^{3}$ value methodology to refine the profitability analysis.

\subsection{Research Methodology}

In this thesis we address two types of problems: 1) knowledge questions, and 2) design problems [33, 34, 35]. Knowledge questions arise when we don't have enough knowledge about a phenomenon in the world while design problems appear when we want to change something in the world so that it suits some actors' goals better. These two types of questions are interrelated in the sense that one can beget the other. To change something so that it suits some actors' goal we may need to increase our knowledge about the actors, their goals and the artifact which we want to change or create. Gaining knowledge about a phenomenon may require designing something which we can use to increase our knowledge about that particular phenomenon.

The research in this thesis consists of three main phases namely: problem investigation, solution design and design validation. In the problem investigation phase we are dealing with a knowledge problem in which we want to acquire more knowledge and improve our understanding of the given problem, particularly concerning collaborative networks, actors and the mechanisms involved in a collaborative network. This can be accomplished via literature review, learn by experience, etc. In this thesis we mostly use literature review for problem investigation. The goal of this phase is identifying those points which we can improve to make them agree better with some actor's goals. In fact, our problem investigation phase is a goal-driven investigation in which there may be no problem experienced but there are nevertheless reasons to change the world in agreement with some goals [35].

The result of problem investigation phase in our case includes the identification of three main challenges/risks which collaborative network designers and actors should deal with. These challenges/risks are discussed in Section 1.2. 
In the second phase we introduce some ideas and methods to address the identified challenges/risks. Most of the thesis is about proposing solutions for three design problems, namely the problem of designing techniques for the three risks identified in Section 1.2. Finally in the last phase we validate our solutions. Design validation is a knowledge task in which we test if the solution would indeed bring actors closer to their goals. For validation we test our findings on some business cases in the real world.

From Chapter 3 to Chapter 8, each chapter discusses a distinct issue and each chapter is self-contained in the sense that each chapter starts with the problem investigation then provides a solution design and finally ends with the validation and conclusion. In addition to that, Chapter 9 provides the validation of the ideas introduced in the thesis by applying them on some extra real world business cases.

\subsection{Thesis Structure}

Chapter 2 introduces basic concepts and notations used in the thesis, such as value modeling, coordination modeling and also game theory. Chapter 3 presents a stepwise method to generate a consistent coordination model based a value model. Chapter 4 discusses a method for the reverse transformation, namely generating a value model from a coordination model. Chapter 5 investigates the automation of the transformations presented in the previous two chapters.

Chapter 6 provides a comprehensive discussion about trust and its role in the business collaboration and then it introduces a method to measure the trustworthiness of business actors in the collaborative network. Chapter 7 introduces a special type of value exchange and then provides a method to analyze the result and the payoff of each actor involved in such a value exchange using the well-established concepts and techniques of game theory. Then, the role of trust on the endurability and profitability of the collaborative network is presented in Chapter 8 and later in that chapter we introduce a method to refine the profitability analysis of a collaborative network by taking the trust relations between business actors into account. Chapter 9 presents some real business collaboration cases and we examine the applicability and usability of our findings on different case studies. Finally, Chapter 10 concludes the thesis by providing an outlook of the contributions of the thesis and also some hints regarding the fu- 
Table 1-1 Thesis structure based on research problems

\begin{tabular}{|c|c|c|c|}
\hline & \multicolumn{2}{|r|}{ Chapter } \\
\hline & & No. & Title \\
\hline \multirow{3}{*}{ P1 } & $\mathrm{P} 1.1$ & 3 & From Value Model to Coordination Model \\
\hline & P1.2 & 4 & From Coordination Model to Value Model \\
\hline & $\mathrm{P} 1.3$ & 5 & Automation of Model Transformation \\
\hline $\mathrm{P} 2$ & $\mathrm{P} 2$ & 6 & Trust and Collaborative Networks \\
\hline \multirow{2}{*}{ P3 } & P3.1 & 7 & Managing Trust in Collaborative Networks using Game Theory \\
\hline & P3.2 & 8 & Endurability and Profitability Analysis of Collaborative Networks \\
\hline
\end{tabular}

ture research. Table 1-1 depicts the structure of the thesis. It shows research problems and those chapters that address each research problem. 


\section{Basic Concepts and Notations}

"Shoot for the moon. Even if you miss it you will land among the stars."

- Les Brown.

To be able to justify an innovative business idea we need some tools and notations to model and present them in a precise and accurate, yet easy to understand way. A business collaboration is a multi-perspective phenomenon and therefore should be modeled and analyzed from different perspectives. The most important aspect of a business collaboration which needs to be clarified for all participating actors is the economic value aspect because, after all, the ultimate goal of every participant in a business collaboration is to gain some economic value one way or the other. The models which we use to justify an innovative business idea should be easy to understand for those who don't have too much technical knowledge yet they should be precise and accurate. One tool for this is $e^{3}$ value which we discuss in more detail in Section 2.1.

The coordination aspect of a business collaboration is another important aspect which needs to be modeled so that the tasks and activities of participating actors and their temporal ordering are clarified. The coordination aspect can be modeled with different tools and notations each of which has its own specific properties and specifications. To avoid details on the internal structure and workings of actors and to focus only on the way that services of different actors communicate with each other, we model this aspect using Business Process Model and Notation (BPMN). We explain this in more detail in Section 2.2.

And finally in Section 2.3 we discuss briefly the concepts and techniques of game theory which we use to analyze the behaviour of participating actors in a business collaboration. 


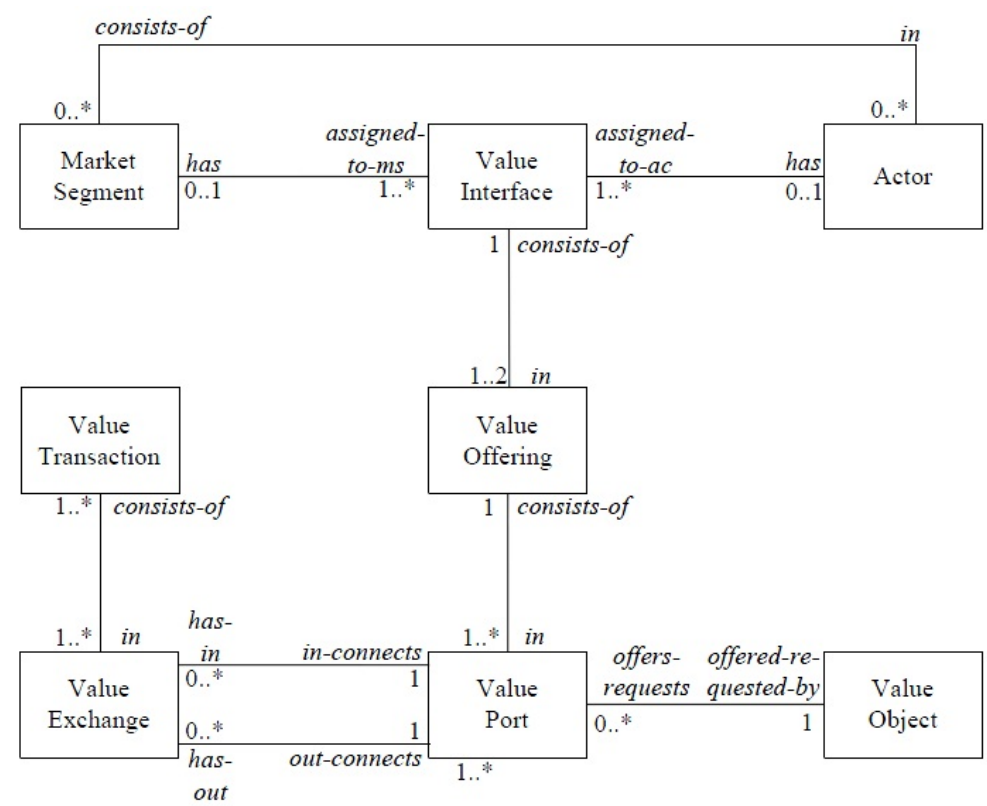

Figure 2-1 Concepts and relations of the $e^{3}$ value ontology (adapted from [27])

\subsection{Value Modeling}

An $e^{3}$ value model consists of a graphic part and a computational part. The graphic part is a diagram and the computational part is a spreadsheet with algorithms that can perform Net Present Value (NPV) estimations for the participating actors in the diagram. In $e^{3}$ value we model a collaborative network as a graph in which the nodes represent economic actors and the edges represent economic value transfers. In addition, an $e^{3}$ value model shows how a consumer need is met by a set of economic exchanges between actors in this web [27, 14, 36, 37]. Figure 2-1, which is taken from [27], shows the concepts and relations of the $e^{3}$ value ontology.

Consider the simple business collaboration shown as an $e^{3}$ value model (Figure 2-2). In this collaboration, Buyer gives Money to Seller and receives Good in return. The Seller, in turn, gives Money to the Transporter and receives Transport. In the following we explain the modeling constructs of $e^{3}$ value using this model:

- Contract Period. A value model describes economic exchanges during a specific period of time, which is called contract period. The contract period should be specified in supporting documentation and the model will be used to analyze economic profitability and sustainability during this period 


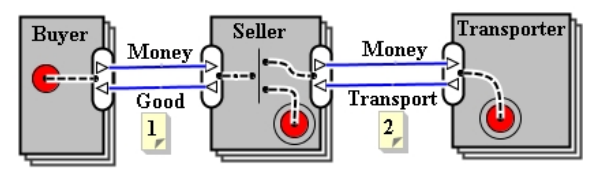

Figure 2-2 A simple value model

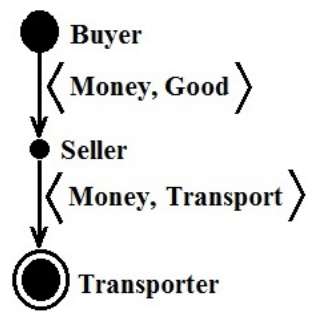

Figure 2-3 A transaction decomposition tree

only.

- Actor. An actor is an independent economic (and often also legal) entity with a specific interest in the collaboration (making profit, increasing utility, earning experience, etc.). The goal of an actor for collaboration is to gain value at the end of the contract period. The concept of value is extensive and it can imply different things. For an enterprise or a business unit value generally means money. However, in some cases value might not directly refer to money, for example an enterprise might collaborate with some other enterprises not necessarily for making money but for gaining some experience. Generally money, increasing utility, gaining knowledge, entertainment, etc. can be regarded as value. Nevertheless, for an enterprise every value boils down to money in the end. In fact, in order to be profitable an enterprise needs at least one collaboration that gives money as value.

In Figure 2-2, Buyer, Seller and Transporter are actors. An actor is depicted by a rectangle, with his/her enterprize or role name. The actor for whom the collaborative network is made to satisfy his needs is called consumer. We represent the consumer need by a bullet placed inside this actor (Buyer in Figure 2-2).

- Market segment. In value modeling terminology the notion of market segment is employed to denote a set of actors who assign economic value to objects equally. In fact, no two actors assign exactly the same value to objects but when we have a large number of actors who play the same role in the collab- 
oration, to have a comprehensible value model, representing all actors as one type of actor instead of representing them all one by one, is inevitable. A market segment is shown as three overlapping rectangles with an explicit name.

- Value Object. Value object is the central concept in value modeling. A value object is a service, product, good, money, experience, etc. that is of economic value to at least one actor and that is exchanged between actors. When two actors exchange a pair of value objects they both think they are gaining value and it is because they value an object differently and subjectively, according to their own valuation preferences [38]. A value object is presented by showing the name of the object nearby a value exchange. In our example value objects are Money, Good, Money and Transport.

- Value Port. An actor uses a value port to provide or request value objects to or from other actors. A value port is a conceptual construct indicating that during the contract period, the actor is capable of giving or receiving the value object. In value modeling we are interested only in the value objects which are being exchanged between actors, not in the internal business processes of the actors. Using value ports we abstract away from the internal business processes. Value ports are represented by small triangles on the edge of the shapes representing actors.

- Value offering. A value offering consists of one or more equally directed value ports and implies that all ports in that offering should exchange value objects, or none at all. It is used to show that an actor wants to offer/receive some value objects in combination rather than separately. A value port is only in one value offering.

- Value Interface. Value interfaces group value ports and indicate atomicity: if one value port in the interface is triggered in the contract period, all of them are triggered in this period. However, the model makes no statement about when this will happen: this has to be specified in a corresponding coordination model. Actors have one or more value interfaces. Value interfaces are represented by oval shapes surrounding the value ports.

By making a bundle of in-coming and out-going value offerings, value interfaces ensure economic reciprocity. Economic reciprocity ascertains duality of value objects, i.e. every value offering should have a corresponding reverse value offering; they always come in pairs. Reciprocity is different from al- 
truism, which is a form of unconditional kindness [39]. How economic reciprocity is ensured is not expressed by the value model. It needs to be addressed by a robust business process design, trust and associated control mechanisms, legal agreements, and sometimes use of technology [27, 40, 41, 5].

- Value Transfer. A value transfer connects two value ports (one outgoing and one incoming value port) of different actors, representing that the actors are willing to transfer value objects in the indicated directions.

- Value Exchange. Value transfers should come in economic reciprocal pairs, which are called value exchange. A value exchange refers to all value transfers between two actors (mostly a pair of value transfers with opposite direction) ${ }^{1}$.

- Dependency Path. In most cases an actor has multiple value interfaces and these value interfaces can be related. A dependency path connects value interfaces of the same actor together, meaning that if one of the value interfaces is triggered the connected value interfaces must also be triggered [14]. A dependency path consists of dependency nodes and connections. A dependency node is a consumer need, an AND-fork (the sign in the actor Seller) or AND-join, an ORfork or OR-join, or a boundary element (the bull's eye sign in the actors Seller and Transporter). A consumer need is the trigger for the transfer of value objects. A boundary element models that no more value transfers can be triggered. A connection is represented by a dashed line.

- Transaction. A transaction starts when the consumer need triggers a value exchange and completes when all the value exchanges connected to that consumer need are triggered ${ }^{2}$.

\footnotetext{
${ }^{1}$ Our definitions of value exchange is different from its original definition provided by Gordijn [27].

Firstly, Gordijn does not define value transfer, instead he uses the term value exchange to refer to what we call value transfer. We believe that it is not intuitive and clear enough, because exchange refers to a bilateral interaction and this conforms with the definition of exchange in Concise Oxford English Dictionary which is:"give something and receive something else, especially of the same kind, in return."

Secondly, Gordijn enumerates four different types of value exchanges (from actor to actor, from actor to composite actor, from elementary actor to value activity, and from value activity to value activity). Except the first type, the other three types are not actually a value exchange and they happen inside the internal processes of actors which we are not interested in. Moreover, with a high level of abstraction we can model all kinds of actors and their internal processes as an actor.

${ }^{2}$ In Gordijn's definition, a transaction can refer to all value ex-
} 
- Transaction Decomposition Tree (TDT). This is a rooted directed acyclic graph with the consumer at the root and other nodes labeled by other actors linked with business transactions (see Figure 2-3) ${ }^{3}$. The graph presents the AND/OR logic of the transactions: each complete path from the root (making a choice at every OR node) to the leaves represents one set of business exchanges that jointly fulfill the consumer need.

Figure (2-3) shows the Transition Decomposition Tree for the value model in Figure 2-2. It is a simple TDT without AND/OR splits or joins. To illustrate a more complex case, suppose that we had two different Transporters (Normal and Special). In that case the two transporters would be linked to the Seller by an OR split in the value model. So, we would have had the two transporters linked to the seller in the TDT with an OR split between them. In that case we could enumerate two different ways of satisfying the consumer need by traversing the TDT from the root to one of the leaves. The temporal meaning of a transaction decomposition tree is that if the need at the root occurs during the contract period, then the transactions in the tree also occur in the contract period, namely to fulfill the need.

All transactions outside the scope of the value model (because they are not relevant to the economic sustainability estimation) are represented by a bull's eye. The bull's eyes represent the model boundaries and are the leaves in the transaction decomposition tree.

Given an $e^{3}$ value model attributed with quantitative estimations (for example, a contract period, the number of consumer needs in that period and the valuation of objects exchanged), we can estimate the revenue of each actor in the specified contract period. This is a first indication whether the model at hand can be economically profitable for each actor. This economic analysis is a supplement for the value model and they all together help the actors understand the business case better. They also help the actors develop a shared understanding of the business case and since it is free of technical details it can be used as a medium of communication between the actors.

It should be noted that the situation modeled in the value

changes between two actors inside the value model. Again, we believe this is not intuitive enough and therefore we have changed the definition.

${ }^{3}$ In $e^{3}$ value this is called a dependency path but for consistency checking it is important to emphasize that this is actually a tree. 
model is an ideal situation in which all actors are supposed to be trusted. In this phase of the modeling we assume that all actors are aware of and undertake their responsibilities and act according to the agreements made in the value model. One might ask what value such a model has while it does not consider the major issues of trust and adverse behaviour. The answer would be that if a business case can not be justified under these simplifying assumption it definitely is not worth adding more details and analyzing it under more realistic assumptions. Besides, trust in a partner in a business collaboration is a risk which some actors might take to reduce the measurements' cost and in the hope of a greater benefit.

However, to operationalize a business case we need to drop this assumption and take appropriate measures to ensure the desired performance of the collaboration. Part of our research in this thesis revolves around this issue and, therefore, it will be discussed in more detail later in this thesis.

\subsection{Coordination Modeling}

In the literature, there are a lot of definitions of concepts and expressions related to business processes. However, in this thesis, we adopt the following definitions provided by Mathias Weske in [42]:

- Business process: A business process consists of a set of activities that are performed in coordination in an organizational and technical environment. These activities jointly realize a business goal. Each business process is enacted by a single organization, but the process may interact with business processes performed by other organizations.

- Business process management: Business process management includes concepts, methods, and techniques to support the design, administration, configuration, enactment, and analysis of business processes.

- Business process management system: A business process management system is a generic software system that is driven by explicit process representations to coordinate the enactment of business processes.

- Business process model: A business process model consists of a set of activity models and execution constraints between them. 
- Business process instance: A business process instance represents a concrete case in the operational business of a company, consisting of activity instances. Each business process model acts as a blueprint for a set of business process instances, and each activity model acts as a blueprint for a set of activity instances.

Business process models are typically created by business analysts and managers to study the performance of a business process, and they can be used to understand, analyze, improve or re-engineer the business process. A business process may include only the processes of one organization (intra-organizational business processes) or the processes of different organizations (interorganizational business processes). The former can be controlled by a central business process management system analogous to a conductor who controls the musicians in an orchestra; therefore, they are also called process orchestration. However, the latter is designed as a process choreography which demonstrates the interaction messages between business processes [42]. In case of a choreography, we don't have a central control system, and coordination is achieved by exchanging messages.

In this thesis, instead of focusing on the details of internal business processes and their realization, we only focus on the overall structure of business processes and the interaction messages which are exchanged between different business processes (process choreography). Therefore we don't stipulate a particular tool or notation for modeling a business process and, in this way, we do not prescribe a certain implementation strategy or platform.

There are a lot of tools and notations for modeling business processes. In this thesis we use Business Process Model and Notation (BPMN), which is widely adopted as a standard for business process modeling [43,44]. BPMN provides a graphical notation based on a flowcharting technique and it is very similar to Unified Modeling Language (UML) Activity diagram. UML is a standardized general-purpose modeling language for object-oriented software engineering [45]. BPMN is easy to understand for all business actors, including business analysts, technical developers and even business managers, and they can use it as a medium for communication. BPMN and UML are both fostered by Object Management Group (OMG) ${ }^{4}$.

There are some other business process modeling techniques such as xBML (extended Business Modeling Language), EPC (Event-driven Process Chain), etc. xBML is used to define the

\footnotetext{
${ }^{4}$ http://www.omg.org/
} 
business processes of an organization based upon a five-dimensional business framework. It depicts a corporation using a system of diagrams based on five "W"s (What, Who, Where, When and Which) and is supported by approximately 55 rules that govern the usage, output and syntax of the language [46].

$\mathrm{EPC}$ is another business process modeling technique in which a type of flowchart is used for modeling business processes. An EPC diagram can be used for configuring an Enterprize Resource Planning (ERP) implementation and for business process improvement [47].

The Web Services Choreography Description Language (WSCDL) is an XML-based [48] language that describes peer-to-peer collaborations of participants by defining, from a global viewpoint, their common and complementary observable behavior, where ordered message exchanges result in the accomplishment of a common business goal [49].

Figure 2-4 shows an example of business-to-business collaboration via interacting business processes. This model only shows the interaction messages exchanged between the participating businesses and the overall structure of the inter-organizational processes, and abstracts from the details of the internal processes. The reason is that enterprises regard internal business processes as an important asset and generally speaking enterprises do not expose their internal processes to the outside world. This means that the properties of the overall business-to-business collaborations cannot be based on the actual detailed local processes run by the enterprises, but rather on the externally visible behaviour and the associated models to represent those processes [42].

\subsection{Game Theory}

Game theory is the formal study of conflict and cooperation. Game theoretic concepts can be applied whenever the actions of several agents are interdependent, i.e. the outcome of an agent's decision depends not just on how it chooses among several options, but also on the choices made by the agents it is interacting with. These agents may be individuals, groups, firms, or any combination of these. The concepts of game theory provide a language to formulate, structure, analyze, and understand strategic scenarios. Game theory is designed to address situations of decision making where several players must make choices that potentially affect the interests of the other players. 


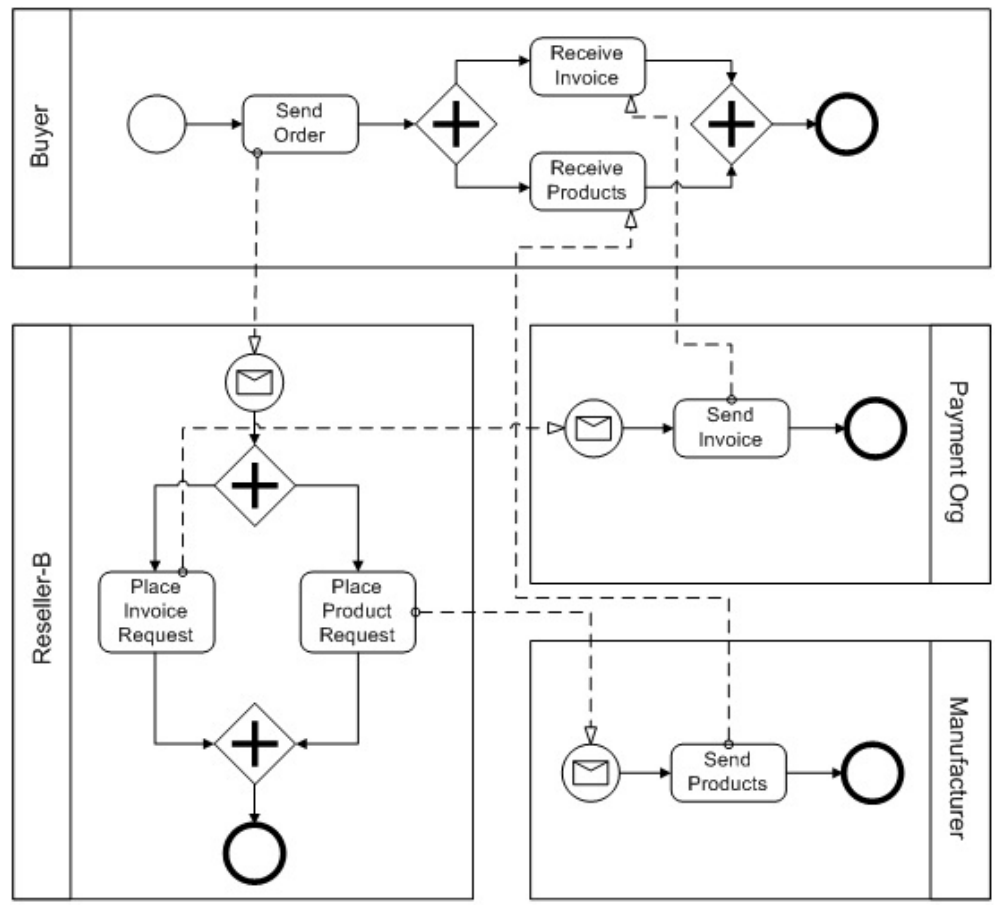

Figure 2-4 An example of business-to-business collaboration through interacting business processes (adapted from [42]) 
Game-theoretic ideas appear in many contexts. Some contexts are literally games. For example, the simple rock-paper-scissors game, which we have probably all played at some time in our lives, can be modeled using game theory. Other settings are not literally considered as games, nevertheless they can be analyzed using the same concepts, such as the pricing of a new product when other competitors have similar new products; deciding how to bid in an auction; choosing a route on the Internet or through a transportation network; deciding whether to adopt an aggressive or a passive stance in international relations; or choosing whether to use performance-enhancing drugs in a professional sport [32], to name but a few. A common aspect between all these examples is that each decision-maker's outcome depends on the decisions made by others, which is the core principle in game theory.

However game-theoretic ideas seem to be relevant in settings where no one is overtly making decisions, e.g. evolutionary biology [32], which addresses evolutionary processes that have given rise to the diversity of life on earth. When a mutant comes to exist from chromosomal alteration, its life depends on the way other non-mutants treat the mutant. Game theory concepts have proven to be useful to analyze the fate of such mutants. Similar kinds of reasoning have been applied to the success or failure of new cultural practices and conventions [32]. In these settings, there is no obvious decision making, however the final result depends on the way that involving agents/actors behave, which relates them to game theory.

In the following we elaborate briefly on the basic concepts in Game Theory:

- Game: A game is "a formal description of a strategic situation" [30]. It is "the study of mathematical models of conflict and cooperation between intelligent rational decisionmakers" [50]. The core concept of game settings is that there are some (at least two) players/agents involved in a situation in which the outcome of the game depends on the behaviours/choices of all involved players.

- Player: A player is an agent who is involved in a game setting situation. A central assumption in many variants of game theory is that the players are rational, i.e. they play in a way which maximizes their own payoff. A rational player is one who always chooses an action which gives the outcome he most prefers, given what he expects his opponents to do.

- Payoff: A payoff is a number that reflects the desirability of an outcome to a player, for whatever reason. The expected 


\begin{tabular}{|c|c|c|c|c|}
\hline & \multicolumn{3}{|c|}{ Player2 } \\
\hline & & Rock & Paper & Scissors \\
\hline \multirow{3}{*}{ Player1 } & Rock & 0 & -1 & $1^{-1}$ \\
\hline & Paper & $1^{-1}$ & 0 & $\begin{array}{ll} & 1 \\
-1 & \end{array}$ \\
\hline & Scissors & & $1^{-1}$ & 0 \\
\hline
\end{tabular}

Figure 2-5 Payoff table for a paper-rock-scissors game

payoff demonstrates the player's attitude towards risk.

- Strategy: In each round of the game, each player's choice is called a strategy. The set of options which each player has for how to behave, determines the player's possible strategies.

- Dominance: Assume that there are two strategies A and B for player $\mathrm{P}$, and given any combination of strategies of the other players, the outcome resulting from $\mathrm{A}$ is better than the outcome resulting from $\mathrm{B}$ for player $\mathrm{P}$. Then strategy $\mathrm{A}$ is said to dominate strategy B. A rational player will never choose to play a dominated strategy and therefore they can be simply excluded from the rest of strategies. In some games, examination of which strategies are dominated results in one single strategy. If this is the case, that strategy is the one that is always played.

- Nash equilibrium: Sometimes, consideration of dominating strategies yields precise advice to the players on how to play the game. In many games, however, there are no dominated strategies, and so these considerations are not enough to rule out any outcomes or to provide more specific advice on how to play the game. A Nash equilibrium recommends a strategy to each player that the player cannot improve upon unilaterally, that is, given that the other players follow the recommendation. Since the other players are also rational, it is reasonable for each player to expect his opponents to follow the recommendation as well. For more details about game theory interested readers are referred to $[30,31,32,50]$.

Figure 2-5 shows possible payoffs for a paper-rock-scissors game. This table is called payoff-table. The units in which payoffs are measured are arbitrary. Like degrees on a temperature scale, they can be multiplied by a positive number and shifted by adding a constant, without altering the underlying preferences they represent. In Figure 2-5, rows represent the strategies of first player and columns those of the second player. Each strategy combina- 
tion defines a payoff pair, like $(1,-1)$ for (Rock,Paper), which is given in the respective table entry. Each cell of the table shows the payoff to first player at the (lower) left, and the payoff to the second player at the (right) top. Note that in this game there is no dominating strategy and also no Nash equilibrium.

In the business collaboration settings, which we address in this thesis, the conflict and cooperation between intelligent rational decision-makers (actors) can be obviously seen. It is also clear that the desirability of the outcome of the collaboration to the actors depends on the behaviours of all participating actors. That provides the suitability of game theory to analyze business collaborations. 


\section{From Value Model to Coordination Model *}

"Genius is one percent inspiration and ninety-nine percent perspiration."

— Thomas Alva Edison (1847-1931) U. S. inventor.

A Collaborative network is a multi-faceted phenomenon and needs to be considered from different perspectives. Two of the most important perspectives are the value and coordination perspective. The former addresses the What questions while the latter deals with the How questions. Value modeling and coordination modeling are both necessary for a good e-business design. In fact, value modeling, with focus on economic sustainability, and coordination modeling, with focus on actor coordination, complement each other.

The main goal of value modeling is to reach agreement amongst profit-and-loss responsible actors regarding the question "Who is offering what of value to whom and expects what of value in return?" In contrast, an important goal of coordination modeling is to reach a common understanding about which coordination

${ }^{*}$ This chapter is based on the following papers:

1) Hassan Fatemi, Marten van Sinderen, and Roel J. Wieringa. Valueoriented coordination process modeling. In the Proceedings of the 8th International Conference on Business Process Management (BPM 2010), Lecture Notes in Computer Science (6336). Springer Verlag. pages 162-177 ([51])

2) Hassan Fatemi, Marten van Sinderen, and Roel J. Wieringa. From business value model to coordination process model. In the Proceedings of the Second IFIP WG5.8 International Workshop on Enterprise Interoperability (IWEI 2009), Lecture Notes in Business Information Processing 38. Springer. pages 94-106 ([52]). 
activities should be carried out, by whom and in which order. These are two different modeling goals, asking for different modeling methods with different constructs [16]. Nevertheless, despite the differences, a value model and its corresponding coordination model should be consistent with each other because they both refer to the same system.

In this chapter we discuss the issue of achieving consistency between value models and coordination models in multi-perspective e-collaborative network design (sub-problem P1.3, Section1.3) and introduce a method to produce consistent coordination models from value models (sub-problem P1.1, Section1.3) in a simple and stepwise manner.

Section 3.1 discusses the value and coordination models and enumerate their differences and similarities. Then, in Section 3.2 a stepwise approach to produce a coordination model from a value model is introduced, and an initial validation of the approach with a real-world example of a collaborative network is provided in Section 3.3. Finally, the chapter is concluded with the discussion and conclusions in Section 3.4.

\subsection{Value Model vs Coordination Model}

To develop a comprehensive understanding of value models and coordination models we first enumerate their differences and similarities and then, based on that, we provide a consistency definition.

\subsubsection{Differences}

Consider the coordination model in Figure 3-3, which is consistent (in a way to be explained later) with the value model of Figure 3-1. There are a number of differences between these two models. In general the conceptual gap between value models and coordination models is caused mostly by the following properties of these two models:

1. Ordering: The key concept in value modeling is value while its counterpart in coordination modeling is time. In an $e^{3}$ value model there is no notion of time ordering at all [14]. Behavior and temporal ordering are beyond the value perspective and are part of the coordination perspective.

2. Time-related properties: From the value perspective, when value $\mathrm{V}$ is transferred from actor $\mathrm{A}$ to actor $\mathrm{B}$, it does not make any difference whether this transfer occurs at once or 


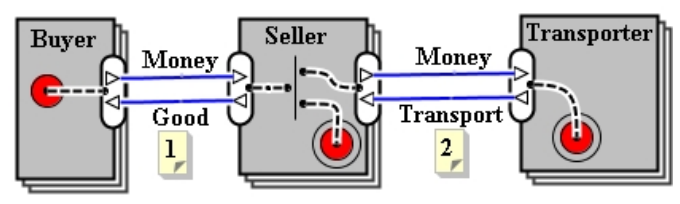

Figure 3-1 A simple value model

in some steps, and also there is no difference between a timecontinuous and time-discontinuous value transfer. In the coordination model all these time-related properties should be determined.

3. Value versus coordination objects: We call objects in the value and coordination models value objects and coordination objects respectively. In a value model every object should be of value to at least one partner, but in a coordination model objects are not included necessarily because they are of economic value to a partner. They can also be included because they help coordinating the activities of the partners (for example, messages). Messages carry/convey coordination objects, namely the information exchanged between the actor processes and used by these processes for coordination purposes.

4. Third parties: A direct value transfer between two partners in a value model does not necessarily imply that there will be a direct coordination object exchange between these partners in the corresponding coordination model. Sometimes a third party will be involved and the path for value object exchange becomes an indirect path for control object exchange. In the example at hand (Figure 3-1), there is a direct value transfer between the buyer and the seller, while the physical transfer of the good that is the subject of the value exchange will require an indirect coordination object exchange between the buyer and the seller involving a transporter.

5. Payment methods: Money transfers are the most common transfers in value models that indicate paying a partner some money in exchange of his/her service or good. A money transfer between two partners in the value model, does not prescribe/impose any payment method. There is a wide variety of payment methods and they may have different corresponding coordination models.

Moving from one type of model to the other requires that the conceptual gap caused by the above factors is bridged. 


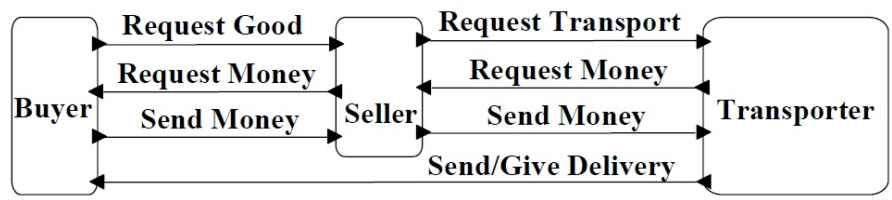

Figure 3-2 All interactions (exchanged messages)

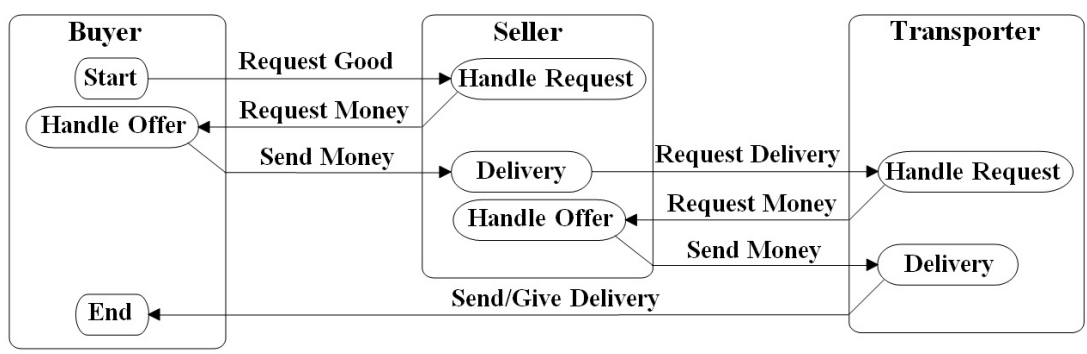

Figure 3-3 Coordination model

\subsubsection{Similarities}

Despite the aforementioned conceptual gap, value modeling and coordination modeling also address some common aspects from which requirements for consistency can be defined. Firstly, they have the same actors/partners. In fact this is a matter of the choice of the designer, and not an inherent property of the models. For example, a designer may choose to represent each actor in the value model by one or more partners in the coordination model, to e.g., reflect the involvement of different departments within a single organization. However, we prescribe that each actor in the value model should have a corresponding partner in the coordination model, because we are not interested (for what it concerns our method) in further structural refinement of these partners. An actor joins a business collaboration only if it earns something of value to itself. Hence, every actor in a collaborative network must perceive some value and therefore will be present in the value and coordination models independently. Secondly, a coordination model has a contract period too, with the same meaning as the contract period of the value model, the actors have agreed to behave in a certain way during the contract period. Finally, each value transaction indicates that something should happen to realize it.

In the coordination model we abstract from internal activities of actors, i.e. from activities that don't involve communication with another actor. In fact, internal business processes are an 
important asset of enterprises and therefore enterprises normally avoid to disclose information about them to the outside world. This means that the properties of the overall business-to-business collaboration must not be based on the internal processes of the participating enterprises, but rather on the externally visible behavior and the associated models to represent it [42].

We add some simplifying assumptions to reduce the complexity of the problem and converge different solutions to a single one. The most important simplifying assumption is that all actors are trusted so that we don't need to consider security mechanisms to mitigate the risk of frauds. In a realistic business model, this assumption needs to be dropped but before building such a realistic model, the partners need to check whether the cooperation is economically sustainable (value model) and practically possible (coordination model) under the assumption that they can trust each other. If economic sustainability and practical possibility cannot be shown under the assumption of mutual trust, it is not worth the effort to check this under the more complicated conditions of lack of trust [22]. In this chapter we therefore make this simplifying assumption, but later in chapter 6 we will drop it. We also abstract from some interactions, like confirmation messages; i.e., we assume that communication is reliable, or unreliable communication can be solved at a lower level with no impact on the considered level. This does not decrease the applicability of our guidelines, because any set of interactions between two actors can be elaborated with more detailed protocol information without creating an inconsistency with the value model.

Under the above simplifying assumptions, for each value transfer a pair of messages (coordination objects) is enough to realize it. This pair consists of a request message and a message referring to the actual value object of the corresponding value transfer.

\subsubsection{Consistency}

In the current line of research, two approaches for maintaining consistency between the value and coordination perspectives are used: (1) informal approaches, that provide a set of guidelines on how to use the value perspective, e.g. for finding a related coordination perspective and vice versa $[8,18,19,20,21]$, and (2) formal approaches, that define consistency rules between perspectives that can be checked, e.g. by model checkers [22, 23, 24]. Our proposal to maintain consistency uses both approaches. We first provide an improved definition of consistency between value and coordination models of a collaborative network, and then we 
introduce a method to design a coordination model from a value model resulting in a consistent pair of models. The contribution of this work is unique because it shows how to move from a value model to a coordination model in a structured and stepwise way using coordination patterns.

The similarities between value models and coordination models motivate the definition of consistency between these models. Zlatev and Wombacher [23] were the first to define consistency between an $e^{3}$ value and a coordination model based on an equivalence of a common semantic model (reduced model that contains the common concepts from two models). Wieringa and Gordijn [22] try to generate correctness formulas for value models based on the correctness formulas provided by the designer for each business transaction. Pijpers and Gordijn [24] check consistency by constructing an intermediate model that captures the physical transfers in a value model, thereby reducing the conceptual gap between value and process models. This physical transfer model can then be checked for consistency with a process model via a reduced model approach. Bodenstaff [25, 26] introduces another definition based on checking the revenue estimation of a value model with the runtime behavior of the coordination model.

Here we first define two concepts and then introduce our definition which integrates and generalizes the original definitions in [22] and [23].

All these approaches make the basic correspondence assumption that each value transfer should correspond to some message exchange in the coordination model and that inversely each message exchange should correspond to some value transfer. Under the simplifying assumptions of the previous Section, except for some special value exchanges which we discuss later, each value transfer corresponds to a request/reply pair. To elaborate this into a consistency definition, we need the concepts of transaction path and execution sequence.

A transaction path in the $e^{3}$ value model is a complete (containing all children of each AND node) and non-redundant (containing exactly one child of each OR-node) path from the root of a transaction decomposition tree ending in the boundary element (bull's eye sign). An execution sequence in a coordination model is a trace from start to end state.

Like the value model, the coordination model is valid in a specific period of time (contract period). We must assume that a domain expert has defined the basic correspondence assumption for each value transaction. The consistency definition then basi- 
cally tells us if a coordination model and a value model respect each others AND/OR logic. The consistency definition is general, i.e. it does not depend on simplifying assumptions. A value model and a coordination model are consistent if:

1. The sets of actors in both models are the same.

2. The contract period of both models are the same.

3. For each transaction path in the value model, there is an execution sequence in the coordination model which realizes the value transfers of that path, and

4. For every possible execution sequence in the coordination model, there must exist at least one transaction path such that the message exchanges contained in the execution sequence represent all the value transfers in the transaction path. Each message in an execution sequence is part of the realization of a value transfer or it exists because it defines a coordination logic.

It is possible that two different coordination models realize the same value model, if they only differ in the coordination logic. This is an informal definition, but it is precise enough to be formalized.

\subsection{From Value Model to Coordination Model}

Several authors have proposed a method to build a coordination model from a value model $[18,19,20,21,8]$. Pijpers and Gordijn [18] proposed a method that makes an intermediate model ( $e^{3}$ transition model) based on the value model by extending it with independent transfers of ownership rights of an object and the actual object itself.

Anderson and Bergholtz [19] proposed a method that starts with a value model and in a number of steps, each value exchange is analyzed and identified as a sub-process of the coordination model. They break value exchanges into components (resource, right, custody, and document evidence).

Wieringa et al. [21] claim that coordination modeling is facilitated by making a physical delivery model first, because the value and coordination model are both views of a network of physical deliveries. They distinguish discrete from cumulative goods and time continuous from time-discrete deliveries. They also specify frequency or duration of deliveries and make a delivery model as an intermediate model on the way to design a coordination model.

In our opinion, these approaches are all too complicated be- 
cause they use intermediate models and/or introduce complicated concepts like ownership right, custody or physical delivery that makes it hard for others to use them in practice. Also, these methods have so far not been tested on other cases than the one they have been developed for. Our proposed method does not introduce these additional models or concepts and is therefore simpler and, as argued at the end of this chapter, easier to generalize.

On the basis of the analysis in Section 3.1, we proposed a stepwise method. The point of departure is an $e^{3}$ value model. For illustration, we explain our method using the $e^{3}$ value model in Figure 3-1.

- Step 1: The first step is identifying the actors of the coordination model. The actors in both value model and coordination model must be the same. Hence, in our example they are buyer, seller, and transporter.

- Step 2: In this step we aim at identifying groups of related value transfers and selecting the most suitable coordination pattern for each group. In other words, we determine the necessary interaction messages which should be included in the coordination model to realize value transactions. A domain expert must indicate which sequence of coordination messages corresponds to each value transfer. Logically, there are only a few possibilities, which are listed below.

- Simple Direct: In this case, when an actor asks another actor to send him a value, the latter replies the former by sending him directly the requested value. This means that the receiver of the request is able to satisfy the requester's need without involving other actors. A simple value model with one value transfer and its realization according to this coordination pattern are shown in Figures 3-4 and $3-5$ respectively.

- Scheduled: There is a special type of value transfer, referred to as scheduled transfer, which doesn't need two messages (coordination objects) in the coordination model. An example of this type of value transfer is scheduled payment in which a partner pays an already determined amount of money for a service/good on already scheduled times. In this case no party asks the other one for paying the money. Hence, in the coordination model we only have one interaction referring to the actual value object. Figure 3-6 shows the realization of the value transfer in Figure 3-4 according to this coordination pattern.

- Direct with arrangement: When an actor asks another 


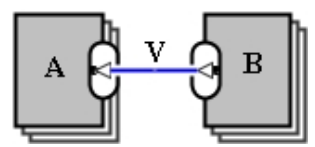

Figure 3-4 Two actors and a value transfer

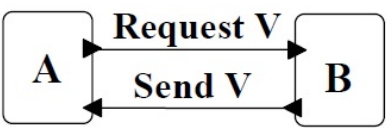

Figure 3-5 Messages in simple direct coordination pattern

actor to send him a value, the latter may, in turn, ask some other actors to send him some values and then reply the former with the requested value. This arrangement can be necessary in case of a preparation or an obligation. In a preparation, the receiver of the request is not able to satisfy the requester's need alone, so he should involve some other actors to play a role. However, in an obligation the receiver of the request is able to satisfy the requester's need alone, but doing so obliges him to make some arrangements (value transfers). An example of a preparation case is ordering raw materials by a factory, and as an example of an obligation is the clearing proprietary rights of a music track by a music provider.

These pre and post requisites (preparations and obligations) are not mutually exclusive and they both can appear in one case and from the value point of view they are the same. A value model and its realization according to this coordination pattern are shown in Figures 3-7 and 3-8 respectively. In Figure 3-8, all the requests are connected to the same AND-split (they execute in parallel), however they can have any ordering. The only implication is that they should be realized before the transaction which is dependent on them.

- Partial: If the request consists of some distinct parts and the receiver of the request is able to satisfy some parts of the request, he might send those parts directly to the

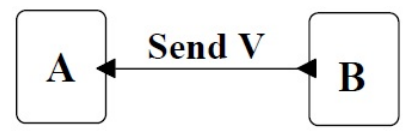

Figure 3-6 Messages in scheduled coordination pattern 


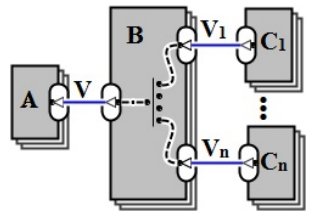

Figure 3-7 Value transfers with AND relation

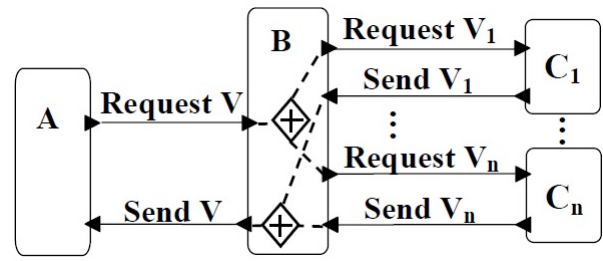

Figure 3-8 Messages in 'direct with arrangement' coordination pattern

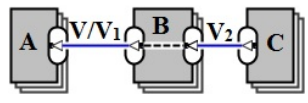

Figure 3-9 A value model

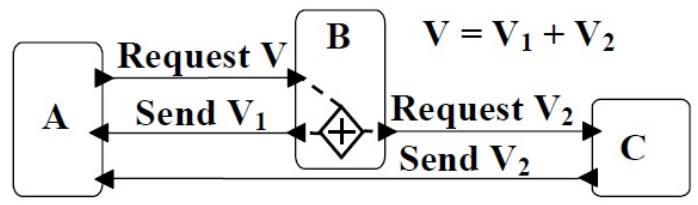

Figure 3-10 Messages in partial coordination pattern

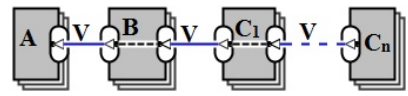

Figure 3-11 A chain of value transfers

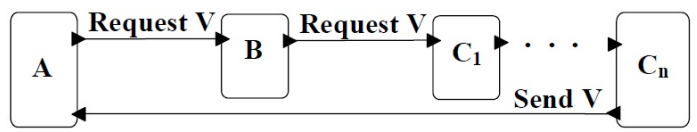

Figure 3-12 Messages in indirect coordination pattern

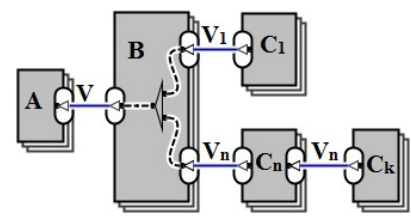

Figure 3-13 Value transfers with OR relation 


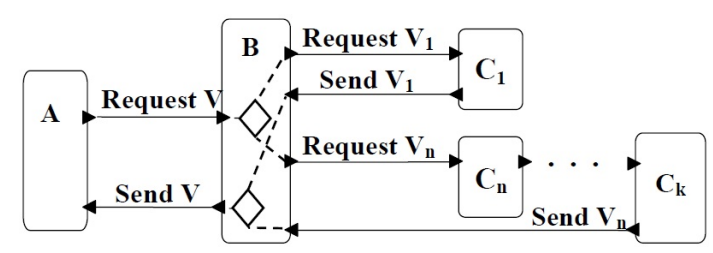

Figure 3-14 Messages with OR relation in combined coordination pattern

requestor and ask (an)other actor(s) to provide the other parts. An example is shown in Figures 3-9 and 3-10.

- Indirect: In this situation, the first receiver of the request plays the role of a mediator by relaying the request to another actor. The first actor in such a relay chain that is able to satisfy the need sends back the requested value to the first requester.

It is also conceivable that the first receiver of the request has the actual requested value but he can not provide the value to the requester or something should be done on it by a third actor before sending it to the requestor. Therefore, he asks another actor to deal with that. For example, a company may transport his products to the customers via a shipping company. This situation may also be modeled in the previous way (Direct with arrangement). Sometimes there is no special distinction between these two situations and either correspondence can be defined. Figures 3-11 and 3-12 show a value model and its realization in indirect coordination pattern.

- Combined: This case is a combination of above situations. Basically any combination of the above cases is possible. Figures 3-13 and 3-14 show one possible value model and its realization in combined coordination pattern. Here the combination is just a matter of juxtaposition.

A value transaction between two actors aggregates two or more reciprocal value transfers. Thus, except for the scheduled coordination pattern, we need at least four message transfers to realize each value transaction in the coordination model. By traversing the Transaction decomposition tree starting from the consumer, different business scenarios and those value transactions which should occur during each scenario are identified.

Back to our example (Figure 3-1), there is only one possible business scenario in which both transactions tagged as 1 and 2 will occur (Figure 3-3). We assume that the domain expert 
has given the following correspondences: the two money value transfers correspond to the simple direct coordination pattern, and the Good and the Transport value transfers make a chain of dependent value transfers. The coordination model after adding appropriate coordination patterns is shown in Figure 3-2 which shows who is causing the transfer of some observable object to whom. Note that this is not an intermediate model and the method is not dependent on it. It is just for illustration and it does not serve any other purposes.

- Step 3: To put the message transfers in a correct order in the coordination model the domain expert has to ask the following two questions regarding each value exchange of the value model:

\section{Who should first send a request to whom? (Which partner initiates?)}

2. In which order the value transfers should be realized?

Using the answers to these two questions we can put the four messages of the value transactions in a correct order. Regarding the first question any of the two actors can initiate the communication, however, here we assume that actor A always initiates the communication by sending the first request message. Regarding the second question, generally if actors $\mathrm{A}$ and $B$ are exchanging two value objects with each other, we would have three different orders in which they can exchange value objects:

1. Actor A first sends its value object to actor B.

2. Actor B first sends its value object to actor A.

3. Value objects are sent in an interleaved way.

To illustrate this point, a simple value exchange and its possible realizations in the coordination model are shown in Figure 3-15. Figure 3-15(a) shows two actors (A and B) that exchange a pair of value objects $\left(V_{a}\right.$ and $\left.V_{b}\right)$ with each other. Figure 3-15(b) shows a coordination pattern in which actor $B$ first sends its value object $\left(\mathrm{V}_{\mathrm{b}}\right)$ to actor $\mathrm{A}$ and then actor $\mathrm{A}$ sends its value object $\left(\mathrm{V}_{\mathrm{a}}\right)$ to actor B. Figure 3-15(c) depicts the other way around.

Figure 3-15(d) depicts a special coordination pattern in which actor $B$ sends part of its value object $\left(V_{b}\right)$ and then actor $A$ sends its value object $\left(V_{a}\right)$ to actor $B$, finally actor $B$ sends the rest of its value object $\left(\mathrm{V}_{\mathrm{b}}\right)$ to actor $\mathrm{A}$. This coordination pattern is feasible only if value object $\left(\mathrm{V}_{\mathrm{b}}\right)$ can be broken into 


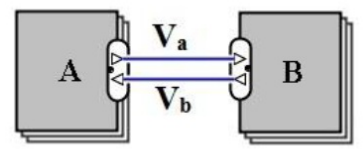

(a) A value exchange

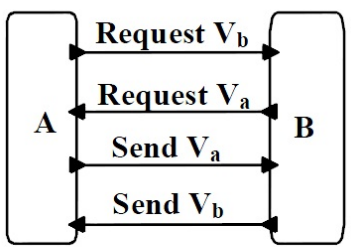

(c) A pair of coordination messages inside the other

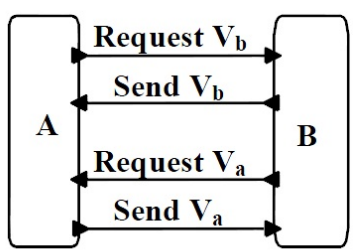

(b) Coordination messages as distinct pairs

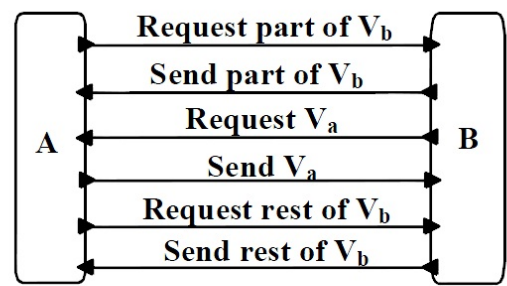

(d) Interleaved coordination messages

Figure 3-15 Possible ordering of coordination messages of a value exchange

some smaller parts. In general, if value objects are breakable to smaller parts, any combination of value transfers is possible. However, for the purpose of our study all those combinations are the same, in the sense that in all those cases both actors, at some point during the value exchange process, send a value object (or part of a value object) to the other actor in the hope that the other actor will act reciprocally and sends back what is promised.

The three different coordination patterns shown in Figure 315 are the only possible correct patterns for coordination messages from value exchange order point of view provided that A initiates the message exchange [7]. All these three different coordination patterns are consistent with the same value model $[7,8]$. In other words, from value point of view they all depict the same situation which is shown in Figure 3-15(a).

Figure $3-15$ is based on the assumption that actor A always initiates the message exchange. This will not affect the generality of the discussion because, the only thing that matters is the order in which value objects are exchanged. If we drop that assumption, there would be other variant(s) for the coordination patterns shown in Figure 3-15. For example, for the pattern in Figure 3-15(b), there would be another variant in which the messages are in the same order as the current pattern except that the message labeled with 'Request $V_{a}$ ' would 
be the first message. However, in both cases the order of the value transfers is the same (first 'Send $V_{\mathrm{a}}$ ' and then 'Send $\mathrm{V}_{\mathrm{b}}{ }^{\prime}$ ). From value point of view, all different variations of the same pattern are equivalent as long as the exchange order of the value objects is fixed [7].

Now back to our case, suppose that we have the following answers to the above two questions regarding value transfers tagged as 1 and 2 in the value model of Figure $3-1$ respectively: 1. The buyer should first send a request to the seller.

2. The payment should precede the Good value transfer and each value transfer is done at once (in one part).

According to these two answers, the ordering shown in Figure 3-15(c), in which A refers to the Buyer and B to the Seller, applies here. There is a subtle difference though; the Seller does not send the Good to the Buyer, instead it triggers the value transaction between itself and the Transporter. Later in this thesis (sub-section 6.4.2), we study the order of coordination messages from the trust relation point of view.

- Step 4: After identifying the necessary interaction messages and putting them in the coordination model in a correct order, we check time constraints of messages. For example, there might be coordination messages that should occur in specific points of time or before a deadline.

- Step 5: In this step we finalize the coordination model by adding necessary administrative activities to each partner (for example, logging activities, confirmation messages, etc.) and link the included interaction messages. Other examples are start and the stop activities (See Figure 3-3). None of these administrational activities correspond to value transfers, but they are needed to make the coordination model work in practice. We refer to this in the discussion at the end of the chapter.

These steps, with the exception of step 2, which depends on human interpretation, can be automated through a CASE tool if we provide it with the necessary information like the answers of the questions of step 3.

The basic question here is: Is the value model generated by our method consistent with its corresponding value model? The first two requirements of the consistency definition of subsection 3.1.3 are satisfied because the method requires that the actors in both models are the same and both models have the same contract period. Requirement 3 of the definition is satisfied because in step 2 and 3 we ensure that each transaction path corresponds to an 
execution sequence. Requirement 4 is satisfied because the only messages added to the coordination model are the ones needed to perform transactions (steps 2 and 3 ) or to control the coordination (steps 4 and 5).

In this way, we can claim that for each transaction path in the value model, which is a set of related transactions, there is an execution sequence in the coordination model and vice versa. Therefore, the models are consistent.

\subsection{Case Study}

To test the applicability of our method to a real-world case, we took an example that deals with the problem of clearing Intellectual Property Rights (IPR). Since 1993, the Ministry of Justice of the Netherlands designated some organizations to take care of the Neighbouring Rights in the music industry. It means that whenever a track is played in a public space with the aim of getting money from it, those organizations must collect fees from such entities making money (IPR users) and distribute these fees to right owners, i.e. artists and producers, etc. (IPR owners). For the sake of copyright issues we have to keep their real names anonymous. We call those organizations IRRS1 and IPRS2.

Currently IRRS1 has contracts with a set of IPR users which are mainly cafes, clubs, restaurants, supermarkets, etc., and charges them based on established tariffs. For each public area the tariff is set according to the size of the public area and the average number of people who visit that public area.

After collecting fees IRRS1 distributes the collected fee between IPR owners according to certain heuristic evidences. Specifically, IRRS1 gets the play lists of the 20 biggest radio stations in the Netherlands and from those lists it measures the percentage of the time that each track is played and then assigns fees to right owners of each track according to the measured percentage of time. This is not a fair way of distributing collected fees because some public areas might not use radio stations and they can play their own list of music tracks. Also there are complains coming from IPR users. They would like to pay only for those tracks they use (pay-per-play scenario). Consequently, it is desirable to count the tracks played by each IPR user. 


\subsubsection{Value Model}

IPR clearing involves two steps: 1) collecting fees from IPR users, i.e. owner of radio stations, bars, discotheques and so on, who play music in public spaces with the aim of getting money from it, and 2) repartitioning the collected fees to Right Owners, i.e. artists, song writers, producers. As described above, IRRS1 is interested in a future business model in which fees are collected on a pay-per-play basis, in which for each music track, a trackspecific network of clearing organizations is composed. This is possible once music is broadcasted over the Internet.

Some results regarding how to model this case study have been already provided [37], however these results only address one of multiple scenarios that can emerge in the music industry, such as new actors performing less or more activities because of market liberalization. There is a new need for IRRS1 for designing a solution based on a pay-per-play scenario because the process of collecting and repartitioning fees is currently based on reports provided by IPR users without any validation. Therefore, IRRS1 would like to know how many times each specific track is played, consequently a new scenario to deal with this problem must be designed, and also market liberalization phenomenon must be taken into account.

Figure 3-16 shows one possible value model of pay-per-play IPR clearing in which BMP (Background Music Providers) delivers a stream of tracks using Internet-based technology for direct playing which is not recordable at Receiver's side. BMP and Receivers both should pay IPR Societies (IRRS1 and IRRS2, see Figure $3-16)$. In this value model actors are:

- Receivers: Receivers are the starting point in the valueobject exchange. A receiver is an actor who wants to satisfy a need (In $e^{3}$ value terminology Receivers are consumers). Since receivers want to use music to get benefits of it, they are IPR users.

- Background Music Providers (BMP): A BMP is an actor who provides specialized background music. For example, an Indian restaurant asks a BMP to provide a special collection of music tracks which are more desirable for its customers. Therefore, whenever an actor requires specific background music, a BMP can provide such background music for a fee.

- IPR Society: IPR Societies are entities performing mainly two roles: 1) collecting fees from IPR users for each played or copied track and 2) distributing the collected fees between the rightful IPR owners of each track. This distribution of fees is 


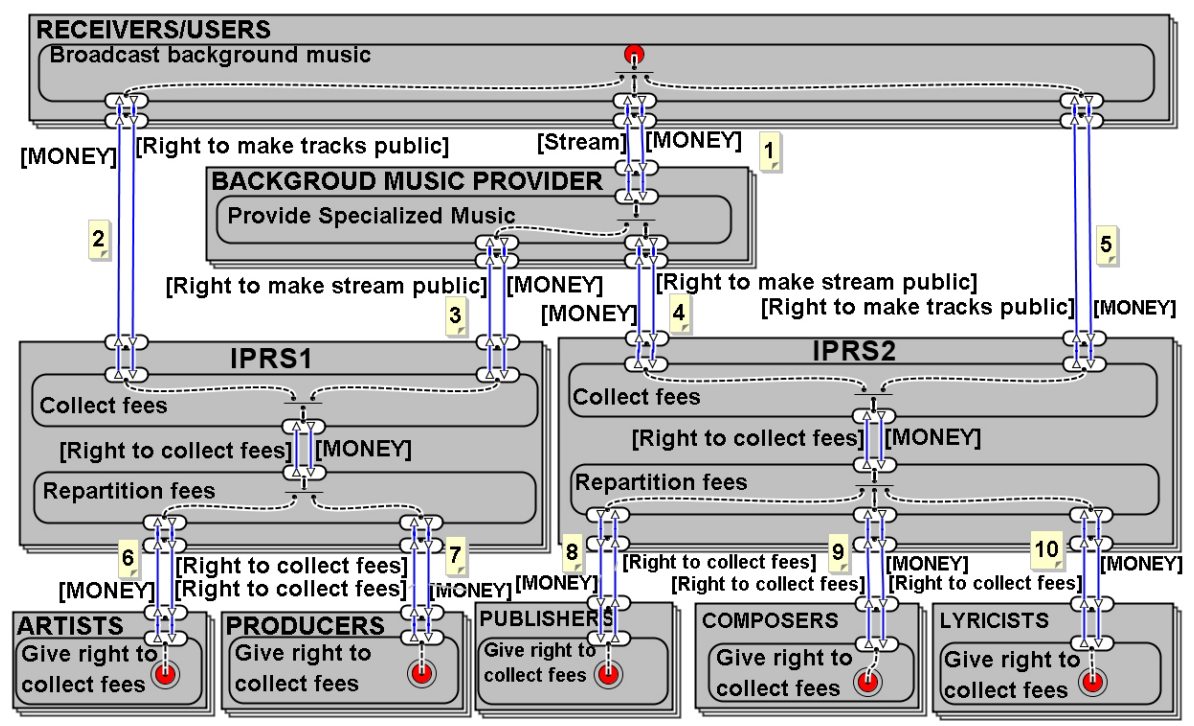

Figure 3-16 Value model of providing music by Streaming

based on track's features, i.e. who the owners are and how many times the track was played or copied. In this case study there are two IPR societies, namely IRRS1 and IPRS2. IRRS1 takes care of IPR rights on behalf of Artists and Producers, while IPRS2 does the same for Publishers, Composers and Lyricists.

- Right Owners: Right owners of a track are those who has been involved in producing it, i.e., write lyrics, play a musical instrument, produce and publish track, etc.

Once the main actors and their roles are identified, it is time to describe how they work together. As mentioned before, receivers are the starting point, they require background music which is provided by background music providers. A Receiver gives a fee to a BMP and gets in return background music. The BMP can provide background music in two ways. It can either deliver a personal copy or a stream of tracks. Personal copies can be considered as a physical device in which the Receiver stores tracks provided by the BMP. A stream is a flow of tracks that BMP delivers to the Receiver using Internet-based technology. So, the main difference between these two ways of providing music is either allowing to store tracks at the Receiver's side or not.

Here we only consider the case in which BMP provides streams of music tracks via Internet. BMP must pay to IPR Societies for making music streams. Receivers have to pay also IPR Societies, 
however they pay for providing music to the public. Paying IPRS2 is about the copyright that the composer and/or lyricist holds, whereas paying IRRS1 is related to the rights of the performing artists and producer.

The process described so far is associated with collecting fees. Therefore the next step is to distribute all those fees. As it is shown in the value model (Figure 3-16), IPR societies distribute fees to those Right Owners who they represent.

\subsubsection{Coordination Model}

We applied our method to this case. The result is shown in Figure 3-17. According to step 1 of the proposed method, actors are the same in both models. Because of space limitations and the high similarity between right owners, we only include one type of right owner representing all of them. Therefore actors are: Receiver/User, BMP, IRRS1, IRRS2, and Right Owner.

Value transactions tagged as numbers 1,2 and 5 match direct with arrangement coordination pattern ( Figure 3-7). However, because the right which the Receiver/User should obtain/clear is dependent on the tracks that BMP provides, first transaction 1 occurs and then 2 and 5. On the other hand, value transactions tagged as numbers 2, 3, 4, and 5 in Figure 3-16 all match the simple direct coordination pattern. In all these transactions a right is exchanged for money. The right requestor (Receiver/User or BMP) should initiate and send money before receiving the confirmation of having the right (coordination message ordering shown in Figure 3-15(c)).

The confirmation sent from BMP to Receiver/User is just for the sake of efficiency. If we remove this confirmation message, the Receiver/User has to wait until the arrival of the stream before being aware of right clearance.

The other transactions (numbers 6 through 10) match scheduled coordination pattern. Hence, we add only two messages to the coordination model to realize each value transaction (one message for each value transfer).

Here we haven't considered the time constraints and durations because the provisioning of music is a simple service for which the duration is already determined. Also the way in which the payments are being done in real life depends on the situations and the agreements between actors, and there is a great variety in this that cannot be all included in the coordination model. For example, instead of paying for each stream in real time, BMP may send a promise to pay to IRRS1 and at the end of the month, it 


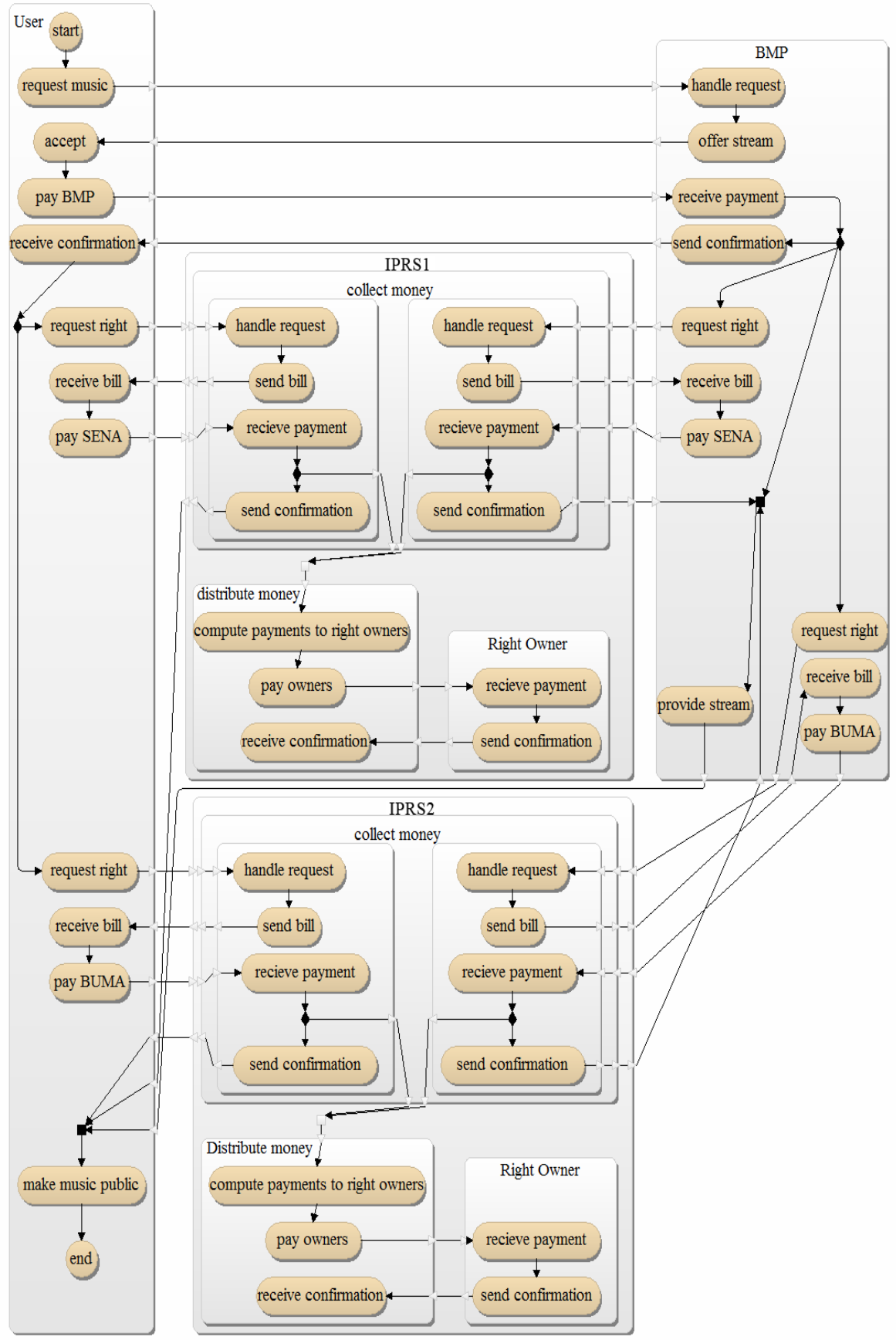

Figure 3-17 Coordination model of providing music by Streaming 
may pay all the payments in batch.

In the last step we include the activities in the coordination model and use them to connect the interaction messages to each other. The two models have the same actors and we assume the same contract period for both. There is one transaction path consisting of all transactions in the model. Hence, there should be only one execution sequence in the coordination model realizing those transactions. This can be easily verified by traveling through the execution sequence in the coordination model starting from the Receiver/User.

\subsection{Discussion and Conclusions}

In this chapter we discussed the problem of how to go from a value model to a coordination model in a stepwise and systematic way. Thanks to the conceptual commonalities that exist between the two models, a method could be proposed that starts with a value model where the main actors and their relationships, in the form of value exchanges, are identified. In a number of steps each value exchange is analyzed and by answering some specific questions a coordination model is designed. The coordination model represents the interactions and interdependencies between the cooperating parties in terms of exchanged messages. We consider a special collection of interactions to realize the value transactions of value models. Based on those analysis we proposed a stepwise method for generating a coordination model from a value model and also checking their consistency in a general and straightforward way.

The proposed guidelines form a simple method that avoids the introduction of complicated concepts like property right, physical delivery, etc. The method does not rely on intermediate models and still it is able to guide the modeler to a coordination model that is consistent with the value model. Because it neither depends on any additional concept or intermediate model nor it does stipulate a special condition or attribute on the models, therefore, we claim that it is more general than existing approaches and it is easily generalizable beyond $e^{3}$ value and BPMN. We have tested our method on earlier cases done by other researchers [18, 21] and observed that our application of the method produced similar results to what was obtained in those cases, and we obtained coordination models that are consistent with their corresponding value models according to our definition for consistency. Because 
our method uses less concepts and does not depend on intermediate models, we hope that our method is both easier to use and applicable to more cases than the other methods.

In step 5 of our method we mentioned that we should add necessary administrative activities to the coordination model to make it work in practice. Some of these activities are related to trust issues, and to get a more realistic result we should drop trust assumptions and enrich the model with the necessary activities. The issue of trust is addressed in Chapters 6 and 7. In addition, more complex coordination patterns will include value transfers realized by multi-step coordination patterns and the inclusion of more sophisticated payment methods.

In general, coordination and value modeling are iterated, since a change in a coordination model may require a change in the value model. For example, adding a trusted third-party in the coordination model requires adding this actor to the value model too. We are aiming at a reverse method that allows those additions to be made in a consistent way. The reverse method is discussed in the next chapter. 
From Value Model to Coordination Model 
Chapter

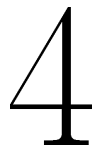

\section{From Coordination Model to Value Model *}

"Men love to wonder, and that is the seed of science."

- Ralph Waldo Emerson (1803-1882).

There are a lot of collaborations in business running for a long time without being really analyzed to see if they still are performing optimally from the value point of view. In addition to analyzing a business collaboration from the value point of view, finding out those activities which can be done differently in a more efficient way or weeding out those activities that do not contribute to the value creation process anymore can also require a value analysis process of a business collaboration. Therefore, after using a business process for some time, it may be worthwhile to revaluate the business collaboration and reengineer it if necessary. To do that we need to design a value model of the business collaborations and analyze it to find out the profitability and sustainability of the collaboration. In this chapter we elaborate on the generation of a value model from a coordination model of a business collaboration (sub-problem P1.2, Section1.3) to analyze the collaboration from the value point of view.

The chapter consists of two sections. Section 4.1 is about generating a value model from a coordination model and Section 4.2 is devoted to discussion and conclusions.

${ }^{*}$ This chapter is based on the following paper:

- Hassan Fatemi, Marten van Sinderen, and Roel J. Wieringa. Valueoriented coordination process model engineering. In Proceedings of the 6th International Workshop on BUSinness/IT ALignment and Interoperability (BUSITAL 2011), volume 83 of Lecture Notes in Business Information Processing. Springer Verlag. pages 41-44. June 2011. [53] 


\subsection{From Coordination Model to Value Model}

As explained in Section 2.1, a value model consists of a graphical part and a computational part. First, we discuss the design of the graphical part of a value model based on a coordination model, and then we address the computational part of a value model.

\subsubsection{Graphical part}

A value model shows the actors participating in the business collaboration and the value objects which they exchange with each other. Hence, the first step is identifying the actors. We assume that the actors are the same in both value model and coordination model. Of course this assumption follows from the assumption that the models are at same abstraction level. Every model can be designed in more detail, and by adding details we might have to break down an actor into more actors and assign specific roles to each of them. However, we are interested in the interactions which take place between the actors, and therefore we abstract from the details of the internal processes inside the actors, and we try to design both models at the same abstraction level. Consequently we assume that both models have the same set of actors.

The next step is identifying the value exchanges and the way they are transferred between the actors. Exchanged messages between actors are either value messages or non-value messages. Value messages are those messages that indicate the transfer of a value from one actors to another. All other messages are nonvalue messages; they are used to coordinate the actions of the actors. We exclude internal activities of actors, because from the value modeling point of view, exchanged messages between actors is the only thing that matters.

Consider the business case shown in Figure 4-1, taken from [54], which consists of a Copier Company that sells and leases copiers to Customer companies. When a customer leases a copier, it must also purchase maintenance on a yearly basis. Figure 4-1 is an inter-organizational process model of this business case that shows which messages are exchanged between actors and in which order.

The first step in designing a value model based on the coordination model shown in Figure 4-1 is identifying the actors. As explained before, we assume both models have the same set of actors (Customer and Copier Company). The next step is identifying the value messages and the relation between them. In this case, messages indicating value transfer are those labeled by service $€+$ lease $€$, copier l, purchase $€$, and copier $p$. The first 

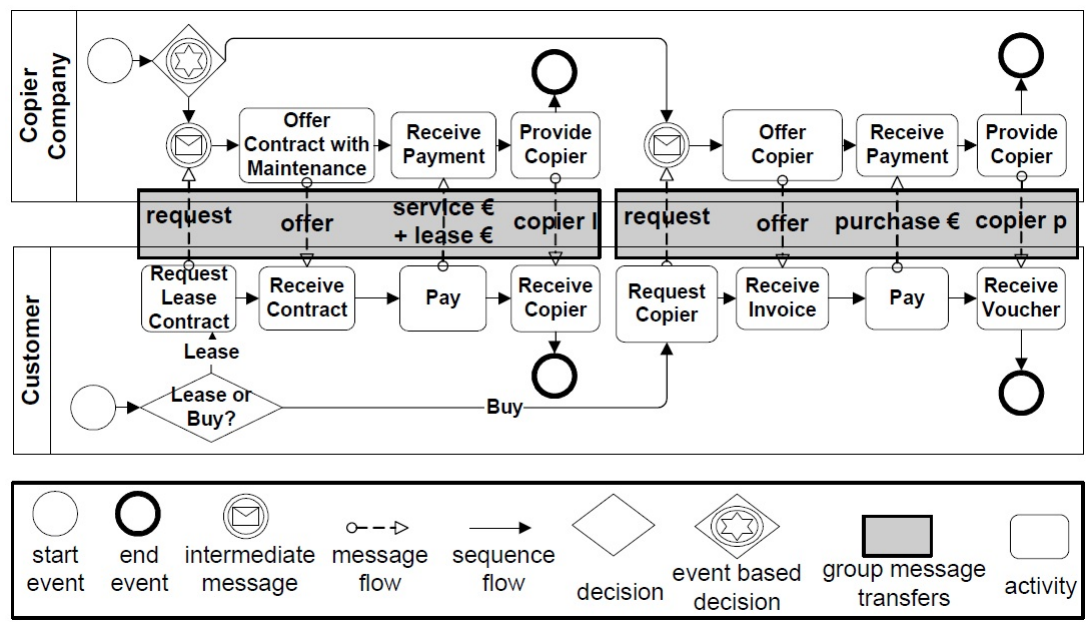

Figure 4-1 A business case in a copier company modeled in BPMN (adapted from [54])

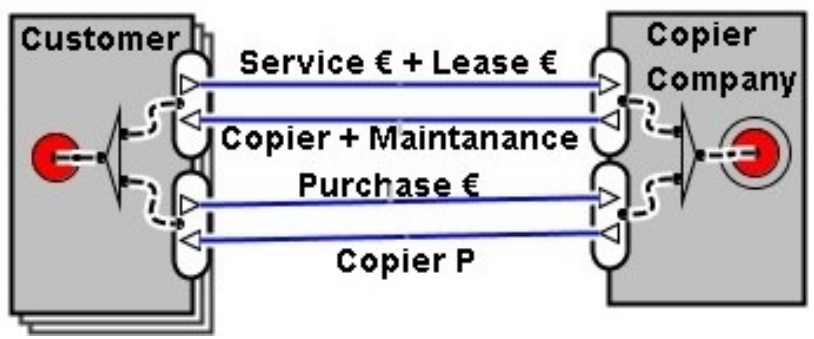

Figure 4-2 The value model of copier case 
and the third one go from Customer to Copier Company and the other two go from Copier Company to Customer. The first and the second value transfer form one value exchange and the other two form another value exchange.

We first add two actors (Customer and Copier Company) to the value model. It is clear that Customer plays the role of consumer (in $e^{3}$ value terminology), therefore we add a consumer need icon (small circle) to Customer and a boundary icon (bull's eye) to Copier Company. Then we add those two value exchanges to the value model. Now, we need to specify the logical relation between the two value exchanges. Looking at the coordination model (Figure 4-1) we conclude that there is an OR-relation between these two value exchanges (a Customer either buys or leases a copier). Hence we complete the value model by adding an OR-Split to Customer and an OR-Join to Copier Company. The value model of this simple business case is shown in Figure 4-2.

As another example consider the business case shown in Figures 4-3 and 4-4, taken from [18]. For generality of our discussion, the coordination model is represented in a UML 2.0 Activity diagram (see www.uml.org). This simple case shows the collaboration between three actors, in which a Buyer buys Good from a Seller. Transporter does the transportation of the Good from Seller to Buyer.

Here, identifying the value-related messages (those labeled with Money and Good) is simple and straightforward, but there is a complication regarding the order and direction of the value exchanges. The two messages labeled with Money are obvious. However, there are two messages labeled with Good, one from Seller to Transporter and the other from Transporter to Buyer, which both indicate the same value object.

Transporter plays a mediator role. Therefore, in the value model we would have a value transfer labeled with Good from Seller (the originating actor of the value object) to Buyer (the final destination of the value object). In value modeling, we model the transfer of value objects between the real owner and the final receiver.

Hence, we add a value object transfer labeled with Transport from Transporter to Seller because Transporter does the transportation service for Seller (the Transporter is being paid by Seller). In this way we represent the duality and reciprocity of the value objects as well. Value reciprocity basically means every value transfer should have a corresponding reverse value transfer; i.e., they always come in pairs. 


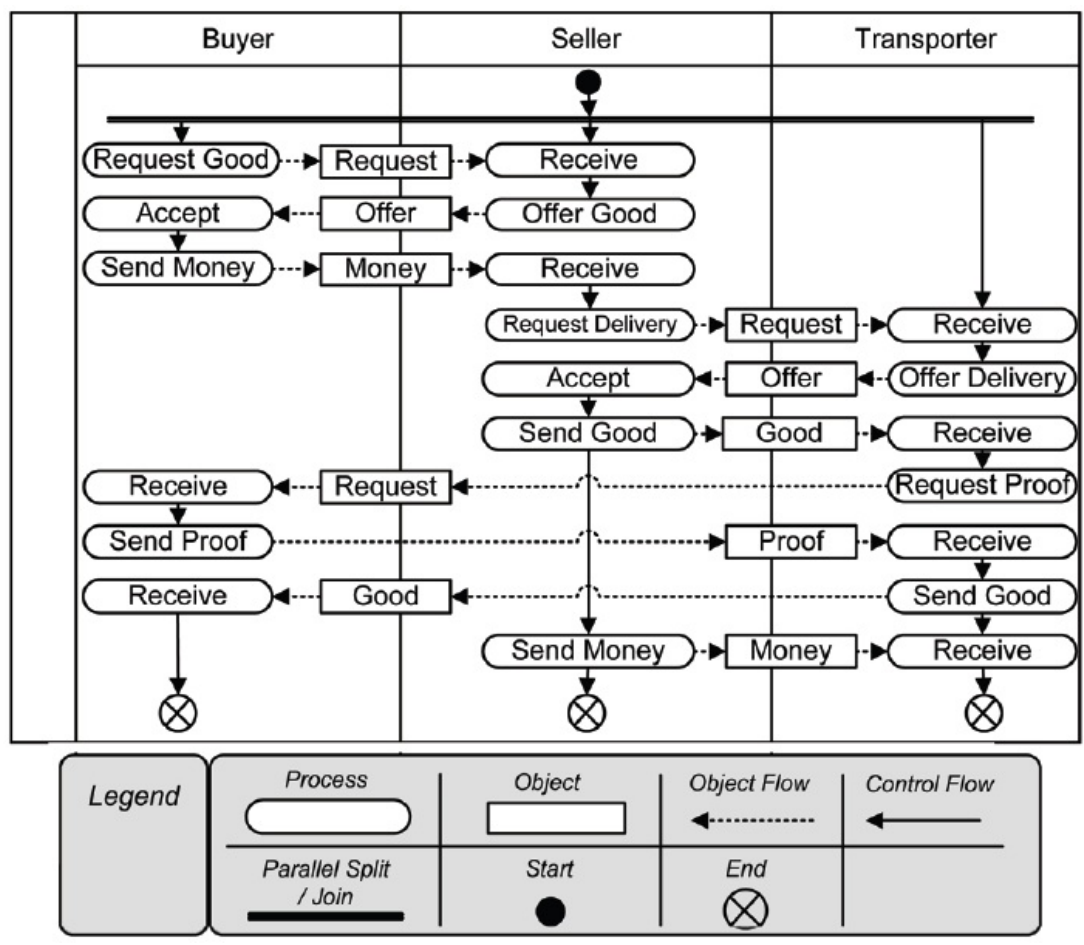

Figure 4-3 A business case in a selling company modeled in UML 2.0 (adapted from [18])

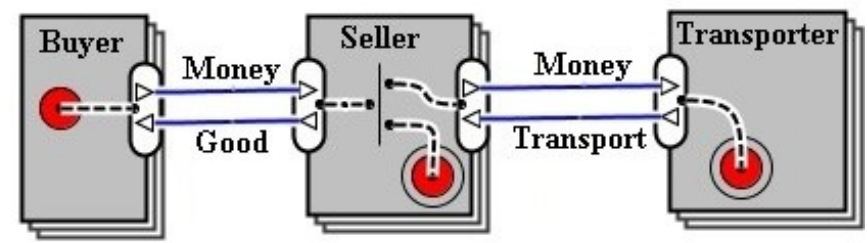

Figure 4-4 A value model 
After adding the value exchanges to the value model we need to specify the actor who plays the role of consumer. In this case, it is also obvious that Buyer plays the role of consumer and the whole business case is made to satisfy the Buyer's need. Therefore we add a consumer need icon (represented by a small circle) to Buyer. We introduce boundary icons (represented by a bull's eye) in both Seller and Transporter. This is the case because part of the money which Buyer pays to Seller goes to Transporter. This also explains the And-Split in Seller. The value model of this case is shown in Figure 4-4.

\subsubsection{Computational part}

The computational part of a value model consists of some tables which show the estimation of the number of value objects which are expected to be exchanged during the contract period and the valuation of each value object in terms of money. Using this information each actor can estimate his/her expected profit during the contract period.

When we design a value model based on a coordination model which already has been implemented, it basically means that we have quite a precise estimations of the number of value objects which are exchanged. In this way, the valuation of value objects is also easier and therefore by analyzing the performance of the running collaboration we can figure out the information which we need to enrich the value model.

\subsection{Discussion and Conclusions}

Once business actors come together and start a business collaboration, any of those actors may want to investigate the productivity of the collaboration and change it if and when needed. This requires knowledge on how good the performance of the business collaboration is from the value point of view.

Here is where value modeling comes into play. Value modeling enables the actors to run some value analysis of the collaboration and this helps the actors develop a shared understanding regarding the collaboration. After developing a value model based on an existing coordination model, we can run a value analysis on the model, and based on the analysis outcome, either regenerate a refined or modified version of the coordination model from the value model $[8,7]$, for example, by using the methods described in the previous chapter, or terminating the existing business col- 
laboration and creating a new collaboration from the scratch.

Note that this chapter presents a preliminary work regarding generating a value model from a coordination model without providing a validation. 
From Coordination Model to Value Model 


\section{Automation of Model Transformation *}

"It is not the strongest of the species that survives, nor the most intelligent that survives. It is the one that is the most adaptable to change."

- Charles Darwin (1809-1882).

In Chapter 3 we discussed about generating a coordination model based on a value model. We introduced a stepwise approach to do the transformation that results in two consistent models. Then in Chapter 4, we talked about the reverse transformation (i.e., making a value model from a coordination model). Now, in this chapter, we want to see to which extent these transformations can be automated. Having a bilateral transformation enables us to track changes in one of the models into the other in a mutual consistent way.

In the previous chapters, we use $e^{3}$ value methodology for designing value models of collaborative networks and we use BPMN for designing coordination models. Here in this chapter we only discuss the automation of the first transformation (value model to coordination model). It starts by representing the value model as a graph and then applying transformation rules on it using a graph transformation tool (Groove) [56].

${ }^{*}$ This chapter is based on the following paper:

- Hassan Fatemi, Marten van Sinderen, and Roel J. Wieringa. Value to BPMN model transformation. In Proceedings of the 12th IFIP Working Conference on Virtual Enterprises (PRO-VE' 11), Adaptation and Value Creating Collaborative Networks, volume 362 of IFIP Advances in Information and Communication Technology. IFIP Advances in Information and Communication Technology 362. Springer. pages 333-340. October 2011. [55] 
The work in this chapter is related to the first research problem (problem P1, Section1.3). We start by a short discussion about model transformation in Section 5.1, then, in Section 5.2 we explain the automation of the value model to coordination model transformation. Finally, we provide the discussion and conclusions in Section 5.3.

\subsection{Model Transformation}

Model transformation consists of the generation of a target model from a source model using some transformation rules. In our case, the transformation can be one-way ( $e^{3}$ value-to-BPMN only) or ideally two-way (reversible). The problem of defining these transformations turns out to be rather complex because of inherent differences between these two languages.

A transformation rule consists basically of two parts: a lefthand side (LHS) and a right-hand side (RHS). The LHS is applied on the source model, whereas the RHS expands the target model. According to [57], aspects of transformation rules are:

- Syntactic Separation. The LHS and RHS may or may not be syntactically separated. In other words, the source and target models may or may not be represented by the same syntax/formalism. If the source and target models are represented using the same syntax/formalism the transformation is called endogenous and if they use different syntax/formalisms the transformation is called exogenous [58, 59].

- Directionality. Transformations may be unidirectional or bidirectional. With an unidirectional transformation, transformation is possible in one direction only. With a bidirectional transformation, transformation can be executed in both directions. Bidirectional transformation can be used to mutually align two models so that the changes in one of the models can be applied automatically in the other and vice versa (i.e., synchronization between models). To have bidirectional transformations we need to have bidirectional rules or define two separate complementary unidirectional set of rules, one set for each direction.

If a transformation rule is a one-to-one function (i.e., every element produced by applying the target model of the transformation rule corresponds to exactly one element of the source model that was used as input to the transformation rule), then the transformation rule can be applied in the reverse direction. 
Transformation rules are usually designed as a function (i.e. given some input in the source model, they produce a concrete result in the target model). However, if different inputs lead to the same output, the inverse of the rule is not a function (i.e., the inversion could enumerate a number of possible solutions). Having invertible rules does not necessarily make the transformation invertible because the invertibility of a transformation depends also on the invertibility of the scheduling logic. Inverting a set of rules may not produce any result due to non-termination. Most of the transformation approaches do not support bidirectionality [57].

- Rule application. In general we may have some rules and conditions which specify the way in which transformation rules are applied. For example, the scope of a rule can be limited to only part of the source model instead of the whole source model. Since there may be more than one match for a rule within a given source scope, we need an application strategy. The strategy could be deterministic, non-deterministic or even interactive. A deterministic strategy could be defined using some standard traversal method (such as depth-first) over the hierarchy in the source model. Examples of non-deterministic strategies include one-point application, where a rule is applied to one non-deterministically selected location, and concurrent application, where one rule is applied concurrently to all matching locations in the source. In an interactive rule application strategy, the system seeks advice from an expert by receiving some inputs whenever it can not deterministically conclude which rule should be applied.

- Rule scheduling. The scheduling aspect can be expressed implicitly or explicitly. In the former the user has no explicit control on the scheduling algorithm, while in the latter the execution order is controlled explicitly. In case of conflicts between rules, a conflict resolution mechanism based on priorities can be used. Rule iteration mechanisms include recursion, looping, and fix-point iteration (i.e., repeated application until no more changes are detected). The transformation process may have several phases, where in each phase only certain rules can be applied.

- Traceability. Transformations may or may not support traceability. Traceability can be useful in performing impact analysis (i.e., analyzing how changing one model would affect other related models), synchronization between models, model-based debugging (i.e., mapping some implementation code back to 
its high-level model), etc.

- Intermediate structures. Some approaches require the construction of intermediate model structures. For example, a transformation process may consist of two phases and at the end of the first phase an intermediate model is generated and the next phase completes the transformation using the intermediate model as input (source model). In such a case the transformations in the two phases might be of totally different natures.

Here we only discuss a one-way transformation ( $e^{3}$ value-to$\mathrm{BPMN}$ ) in which we present the source model as a graph and generate the target model by applying transformation rules to the source graph. Rules can have different priorities and the tool keeps track of the applied rules so that we know in which order they have been applied. The transformation of an $e^{3}$ value model into a BPMN model consists of two phases, the first phase is done by Groove automatically and in the second phase it involves an expert who enriches the automatically generated model by adding some details to the model.

\subsection{Automation of Transformation}

There are satisfactory solutions for transforming models to text/code (such as template-based approaches), but this is not the case for transforming models to models. Our model to model transformation is a graph-based-transformation approach. Graph-basedtransformation approaches are powerful and declarative, but complexity is still a major challenge in those approaches and it limits their applicability. The complexity of graph-based-transformation approaches is due to the non-determinism nature of their scheduling and application strategy. Therefore the termination of the transformation process and the rule application ordering need particular scrutiny [57].

\subsubsection{Transformation Automation using Groove}

We model the value model shown in Figure 3-16 in Section 3.3 as a graph in Groove (Figure 5-1). Each AND and OR node is connected to its appropriate actor's node with an edge labeled as 'r'. They specify the logical relation (AND or OR ) of the incoming and out-going edges to/from a node. To understand the graph more easily we can think of each actor node and its AND and OR nodes as one integrated conceptual node. In this way, 
the model indicates that the Receiver has three value exchanges with IPRS1, BMP and IPRS2 and these value exchanges have an AND relation with each other, i.e. they make a unit of exchange which means either all of them occur or non. IPRS1, on the other hand, has two value exchanges with Receiver and BMP with an OR relation between them. This means that when IPRS1 receives money either from Receiver or BMP, it should distribute the money to the appropriate right owners.

The graph shown in Figure $5-1$ is the starting point in the transformation process. To make a BPMN model from it, we need to model the transformation rules and apply them on the starting graph. Two transformation rules are shown in Figure 52. The rule shown in Figure 5-2(a) expresses that if there is a pair of edges (value transfers) namely ' $x$ ' and ' $y$ ' between two distinct nodes (actors), delete those two edges and add four nodes labeled as 'Send' and 'Request' connected to those two nodes with edges labeled as ' $x$ ' and ' $y$ '. To prevent the recursive application of this rule on the newly added nodes, we need to specify in the rule that the two main nodes should not be labeled as Send or Request.

Similarly, the rule shown in Figure 5-2(b) expresses that if there is a single edge (value transfer), namely ' $x$ ', between two distinct nodes (actors), delete that edge and add a node labeled as, Send, connected to those two nodes with edges labeled by 'x'. To prevent application of this rule on the fake edges labeled as ' $r$ ' it is stipulated that the edge between the two nodes should not be labeled ' $r$ '. Again, to prevent the recursive application of this rule on the newly added node, we need to specify in the rule that the two main nodes should not be labeled as Send or Request.

The first rule has a higher priority and the tool applies it first until it has no more matches. Then the second rule is applied. If we don't specify priority for these two rules they conflict with each other. Then, in the case of a pair of value exchanges between two actors, instead of applying the first rule, the tool may apply the second rule twice.

The final graph resulting from the application of the above two transformation rules is shown in Figure 5-3. The resulting graph shows all the necessary message exchanges between the actors and the logical relation between the in-coming and out-going messages to/from an actor's node. It is worth mentioning that there is no special temporal ordering between the messages in this graph. For example, the Receiver has 6 outgoing and 6 incoming messages but without a special temporal ordering. Therefore, the resulting graph is a communication model (a message transfer model) rather 


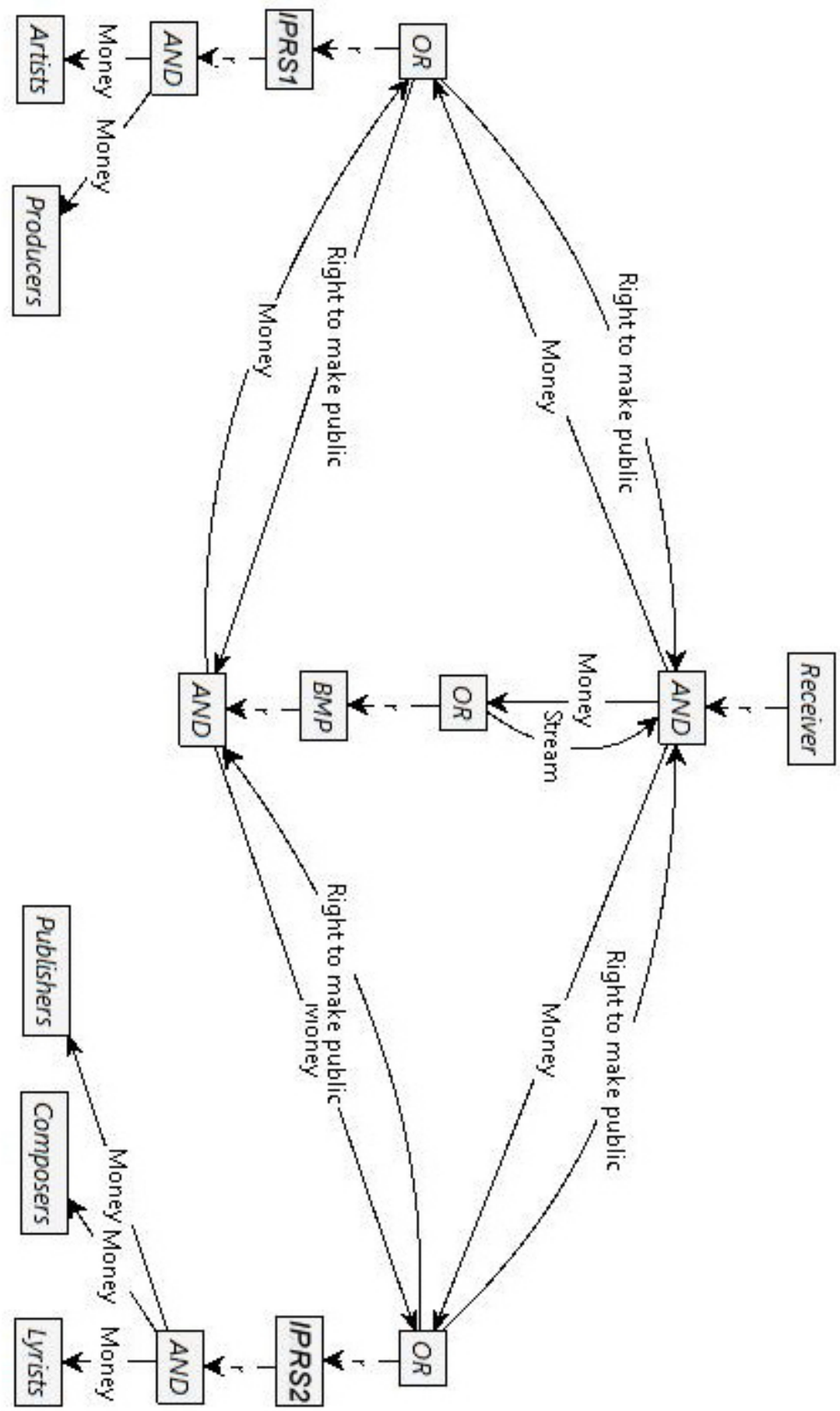

Figure 5-1 Start graph for value model of providing music by streaming 


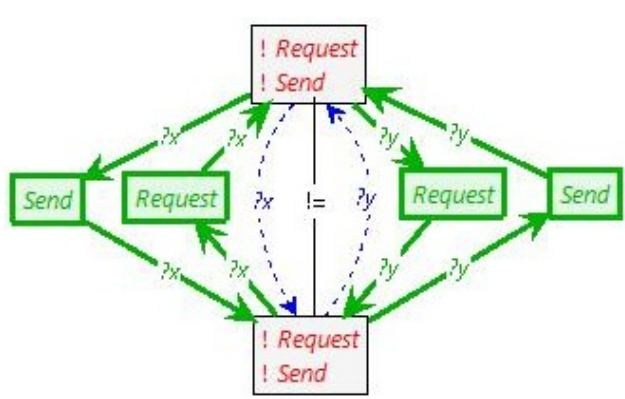

(a) A pair of related value transfers $(x, y)$

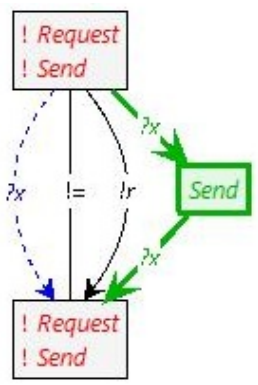

(b) A single value transfer (x)

Figure 5-2 Two transformation rules modeled in Groove

than a coordination model.

\subsubsection{Making the Coordination Model}

As we mentioned above, the result of the transformation process (Figure 5-3) is not a coordination model but rather a communication model that shows all the necessary message transfers between the actors. It also shows the logical relation between all incoming/outgoing messages to/from an actor's node. To make a coordination model we need to add temporal ordering to the model, i.e. indicating the order in which the messages are exchanged between the actors.

The temporal meaning of the dependency path is merely that if the consumer need is triggered in the contract period, then the connected transactions are also triggered in the contract period. The dependency path actually represents the structure of the profitability computations, not a process that coordinates the transactions. Many different coordinations are compatible with a single value model.

Putting messages in a correct and meaningful order needs expert intervention and cannot be automated using the value model only. We need to supplement the value model with more information. Some of the messages indicate the transfer of value from one actor to the other, for example the Send message labeled as 'Money' from Receiver to IPRS1 and the Send message labeled as 'Right' from IPRS1 to Receiver imply the transfer of money and a right clearance respectively. The order in which these value objects are exchanged between actors depends among others on the trust relations between the actors. If, initially, Receiver sends the money to IPRS1 in the hope that IPRS1 acts reciprocally 


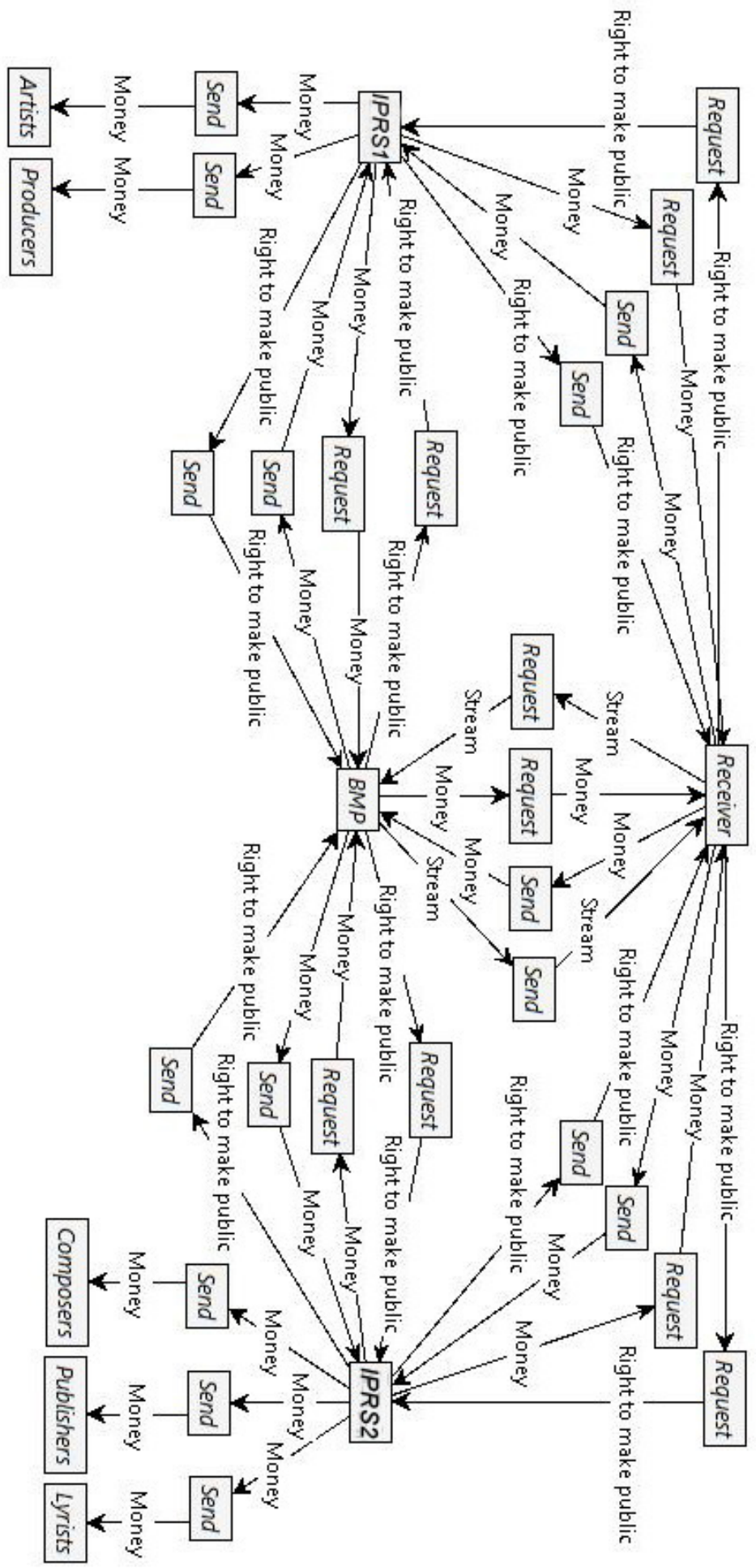

Figure 5-3 Final graph resulting from the above two transformation rules 
by returning the requested rights, it means that Receiver trusts IPRS1. Otherwise, if IPRS1, before receiving the money, sends the requested right to Receiver in the hope that Receiver will pay later, it means that IPRS1 trusts Receiver. The third scenario, in which both IPRS1 and Receiver need to trust each other at some point in time, is the case in which, initially, Receiver pays not all the money but part of it to IPRS1 and then IPRS1 sends the requested right to Receiver and finally Receiver sends IPRS1 the remaining of the payment.

If we had the trust relations between the actors or the way in which they want to transfer the value objects, then we could accordingly determine the temporal ordering of messages in the coordination model. This will be discussed in more detail in the next chapter.

\subsection{Discussion and Conclusions}

According to [16], value models and coordination models can have different actors. This happens only when the two models are of different granularity with respect to actors. By abstracting from the sub-actors and the internal activities inside the actors, both models can always be designed using the same actors. In what follows we assume that this has been done.

One of the main remaining differences between value models and coordination models is type of objects exchanged by actors $[16,7]$. In a value model every object should be of value to at least one partner, but in a coordination model objects are not included necessarily because they are of economic value to a partner. They can also be included because they help coordinating the activities of the partners. The application of the transformation rules covers this difference by adding all the necessary messages to the model regardless of whether they are of economic value to a partner. In this chapter we use a pattern with a minimal number of messages (a pair of 'Request', 'Send' messages) to realize a value transfer. In general, patterns which include more detailed messages can be used. In fact, these basis message types can be complemented with others that are necessary for achieving certain communication properties (e.g., reliability). Generating a coordination model based on a value model by replacing value patterns in the value model with counterpart coordination patterns in the coordination model is discussed in Chapter 3 .

As a difference between value models and coordination mod- 
els, [16] mentions a special case in which there is a value exchange (e.g., experience, entertainment or pleasure) that has no associated direct physical or information flow in the coordination model. We think all value transfers, and all objects in a coordination model, are physical. In a coordination model, we abstract from the physical world and represent the manipulation and transfer of information, but information is implemented physically. But this physical implementation (on paper, as electric signals, dots on a screen, etc.) is not relevant and not insightful here, so we abstract from it. In a value model, all transfers are physical too: on paper (money, books, pictures, etc.), as sound (music, spoken information, etc.). Therefore we think this special kind of value exchanges are not real value exchanges. Instead they are the cause or effect of another value exchange. For example, assume that there is an online radio station that charges some fee if you want to listen to its music. In this case there is one obvious value transfer from the user/listener to the radio station which is 'Money'. However the reciprocal value object may be modeled as 'entertainment' in the value model. If so, applying our pattern does not yield an appropriate result. Nevertheless we think 'entertainment' is not a value transfer but it is the effect of a value transfer, namely 'Broadcasting Music'. Therefore, in this case 'Request'and 'Send' messages are indicating asking and giving permission.

The other main difference between value models and coordination models mentioned by $[16,7]$ is the notion of temporal ordering. In an $e^{3}$ value model there is intentionally no notion of time ordering at all [14]. Behavior and temporal order are beyond the value perspective but are part of the coordination perspective.

$E^{3}$ value models have a value reciprocity concept which basically means that every value transfer should have an associated value transfer in the reverse direction. This concept has no associated counterpart in the coordination model. $E^{3}$ value includes a computational part which enables the actors to do a profitability analysis, which also has no associated counterpart in the coordination model. 
Chapter

\section{Trust and Collaborative Networks *}

"Research is the transformation of money to knowledge. Innovation is the transformation of knowledge to money."

- Hans Meixner.

In this chapter we discuss the role and impact of the trust relations between participating actors in a collaborative network on the coordination model of the collaboration. The assumption that business actors act trustfully is often not useful in practice (since there are malicious actors). Here, we consider collaborative networks from a trust point of view and introduce an approach for measuring the trustworthiness of the actors participating in a collaborative network.

This chapter addresses the second research problem (problem P2, Section1.3). To address that research problem we propose a method to model the trustworthiness of actors involved in a business collaboration so that actors can develop a better understanding regarding the trustworthiness of their partners in the collaboration and make a more stable and durable collaboration.

The chapter starts by an introduction in Section 6.1. Next, a discussion about trust and its specific interpretation in this thesis

${ }^{*}$ This chapter is based on the following papers:

1) Hassan Fatemi, Marten van Sinderen, and Roel J. Wieringa. Trust and business webs. In the Proceedings of the 15th IEEE International EDOC Conference (EDOC 2011), pages 114-121, Helsinki, Finland, 29th August - 2nd September 2011.( [60])

2) Hassan Fatemi, Marten van Sinderen, and Roel J. Wieringa. A Trust Ontology for Business Collaborations. To appear in the Proceedings of the 5th IFIP WG8.1 Working Conference on the Practice of Enterprise Modelling (PoEM), 2012. ([61]). 
is provided in Section 6.2. Then, an ontology of trust in business collaboration is proposed in Section 6.3. After that, we introduce a method to calculate and manage the trustworthiness of business collaboration partners in Section 6.4. Section 6.5 provides a discussion regarding the proposed method and Section 6.6 is devoted to related work. Finally, the chapter ends with a conclusion in Section 6.7.

\subsection{Introduction}

Trust is a ubiquitous phenomenon in everyone's life. For example when we cross a street we trust the drivers to a certain extent that they follow the traffic rules. As Luhmann [62] indicated, trust reduces the complexity of interactions. Trust exists inherently and latently in all our actions that we might even not be fully aware of that. Most of the times people take trust for granted and that is the reason why it is overlooked in many cases. Nevertheless trust has a major impact on our decisions.

In business settings, trust plays even a more important role because in contrast to the social settings, in business settings a misplaced trust might result in financial loss and after all financial profit is the main thing that matters in business settings. Trust is inevitable and in doing any business activity actors need to trust some other actors. As Kenneth J. Arrow [63, page 24] pointed out without trust no market could function and there is an element of trust in every transaction. Hence, in any business collaboration, business actors need to identify the trust factor and evaluate the financial risks and consequences associated with it and be fully aware of them while making a decision.

The collaboration partners participating in a collaborative network want to be sure that participation in the collaboration is economically rational and sustainable before specifying the coordination process of the collaboration. Value modeling does not consider the trust aspect of the collaboration therefore value models abstract from trust-related information. In other words, while value modeling, we assume that all partners in the collaborative network behave in accordance with the rules and promises expressed in it (collaboration partners do not act opportunistically), because otherwise the collaboration does not serve the purpose of all participants.

If we assume that the partners in the collaborative network can be trusted to behave according to the value model, profitability 
analysis of the collaboration over a period of time can be done by first estimating the frequency of value exchanges between actors in that period, and the value of each exchange in terms of money, and then calculating the net amount of money which each partner earns out of the collaboration. However, the risk in any business network is that a partner will not behave according to the value model and act in favor of its own goals, to the detriment of other partners' goals. Each partner will assess this risk and determine a trade-off between the cost of detective and preventive measures (e.g. replacing the distrustful parties with more trusted ones, adding trusted third-parties, contracts, additional checks, monitoring by independent third-parties, etc.) to avoid opportunistic behaviors, and the risk of simply trusting others by assuming that they will not act opportunistically. Nevertheless, for doing any business in the real world, trust is crucial for the success of the business, because, after all, we need to trust at least some actors, such as a bank or other trusted third-parties. This forces a business to take appropriate and sufficient measures against those who it does not trust (i.e. those who may not live up to their commitments).

The purpose of this chapter is to model trust explicitly as a key component of a collaboration between parties who will benefit from the collaboration, but who must also worry about possible opportunistic behaviors of some of the partners. We want to provide the collaborative network designers with a method as a tool to assess the trustworthiness of the actors participating in the collaborative network, and to use this assessment to redesign the value model and/or design the coordination process and IT architecture in such a way that the remaining trust assumptions take an economically acceptable risk. The contribution of the chapter is to introduce an initial model for measuring the trustworthiness of the actors participating in a collaborative network based on their collaboration experiences. We aim at:

1. Allowing the collaborative network designers to incorporate an initial assessment of trust in the value model design process, and

2. Providing a technique for real-time monitoring of the behavior of partners in the collaborative network. This allows a business to gauge its actions accordingly and consider redesigning the collaborative network if and when needed.

Vera Kartseva [40], in her thesis, introduces an initial work on how to redesign a value model so that untrustworthy behaviors are kept under control. Later in chapter 9 we discuss about Kartseva's 
research and the relation between our work and her work.

\subsection{Trust}

Trust is a ubiquitous phenomena and it has been defined in different types of studies. Several connotations of trust can be found in social sciences [64] such as sociology [65], psychology [66], and philosophy [67]. Proposed definitions differ from each other to the extent that Lewicki and Bunker compared trust to the story of blind men and the elephant [68]. Each man perceived the elephant ("trust") to be something different (e.g., a rope, a wall, a pillar, a solid pipe), because each of them felt a different part, but only one part of the elephant (e.g., the tail, the side, the leg, the tusk, respectively). They each thought the elephant was what they felt because they were unable to see the big picture of what an entire elephant is like [64].

Despite the extensive study of trust in social sciences, the formalization of trust from a computational perspective is a new trend. Recently, we are witnessing an enormous increase of interest in modeling trust, mainly driven by the advent of the Internet and electronic commerce.

\subsubsection{Trust and Business}

The view that business requires some certain moral codes is nothing new. Adam Smith already believed that morals mattered [69]. More recently, Francis Fukuyama [70] described economic networks as fundamentally based on reciprocal moral obligations, a generalized trust among unrelated people [71]. Kenneth J. Arrow [63, page 24] pointed out that " . . . ethical elements enter in some measure into every contract; without them no market could function. There is an element of trust in every transaction". Tapscott et al. [5] observe that "Trust is the sine qua non of the digital economy."

There are many uses and definitions of the word "trust" in business and popular press [72, 73], so it is helpful to clarify how the term trust will be used here. According to the ITU-T X.509 [74, Section 3.3.54], trust is defined as follows: "Generally an entity can be said to 'trust' a second entity when the first entity makes the assumption that the second entity will behave exactly as the first entity expects."

For our purpose the most useful definition is the one that views trust as the willingness to take risk: Michalos [75] wrote that "It is 
enough to think of trust as . . . to allow oneself and perhaps others to be vulnerable to harm in the interests of some perceived greater good." Johnson-George and Swap [76] asserted that "willingness to take risks may be one of the few characteristics common to all trust situations." In this thesis, we will follow Michalos' definition of trust because a value model demonstrates a situation in which a group of business partners join together and take the risk that their partners may not honor their commitments in the interest of some perceived greater good.

Hence, we define trust as the belief a business actor has that the other party will act according to the promises and obligations expressed in the value model by acting reciprocally, i.e. the other party returns something of agreed value for what it gets (e.g., goods in return of money). Distrust is defined as the fear of opportunistic behavior from partners, thus the fear for breaking the reciprocity of trust-based interaction. So stakeholders must reason about the trustworthiness of others, in order to decide which other parties to collaborate with. We merely want to provide a tool for decision support, not guidelines for when to trust a partner. The question to be answered is: how does a business partner know whether other partners will honor their commitments?

\subsubsection{Trust and Electronic Commerce}

The role of trust in electronic commerce is vital and much more important than the role that it has in traditional business because in brick and mortar business the partners have a face-to-face contact with each other, and this helps them know each other better. Therefore trusting a partner in this situation is less challenging compared with the situation in e-commerce in which commercial trade is carried out via the Internet or other on-line computer services. We can be reasonably sure that trust relations in e-commerce interactions will not be deep, and as Houston [71] pointed out the biggest risk of doing business "on Web time" is, indeed, being exploited more easily as work relationships diminish.

In e-commerce, a business actor has to do business with some software agents who are acting on behalf of some business actors. In those circumstances, the issue of trust is twofold. First the business actor needs to authenticate the software agent and be sure that the agent is not fake and it represents a real legal business actor, and then the trusting business actor should access the trustworthiness of the business actor who the agent represents. We do not deal with the fist issue and we assume that the business actors know the identity of those they collaborate with. 
Now the question is "In which ways can trust be built up in the digital economy?" One widely used suggestion is to rely on reputation. In fact a business actor trusts its partner only if it has enough knowledge regarding its skills, motivation, and attitude. One way to acquire such knowledge is through experience (collaboration through repeated interactions). By acquiring enough knowledge regarding their partners, business actors aim at predicting the future performance of their partners. This is the approach that we adopt in this chapter.

If business partners could predict the future performance of their partners, they could gauge each other's behavior. This forces the business actors to guard their reputation because otherwise if a business actor becomes known as a shirker or deceiver, his opportunities for future productive dealings will be lessened [71]. Disseminating the information regarding the collaboration experience on Internet is an extraordinary medium by which business partners can intimidate their partners from acting opportunistically. Therefore Internet can be seen as a double-edged sword in e-commerce: on one hand it facilitates the collaboration and decreases the transaction cost dramatically, on the other hand it makes business actors withhold themselves from involving into a collaboration if they are not going to be trustworthy and responsible.

The problem we are dealing with in this chapter is trust assessment in fixed collaborative networks, i.e. the partners are known, except those in a market (e.g. consumers). Our innovation is that, unlike current reputation-based trust assessment methods, we assess the trustworthiness of an actor (e.g. the seller of product $\mathrm{X}$ ) using deep reputation assessment which stem from the performance of the actor in the whole collaborative network, rather than just assessing its behavior with his customers (of that particular product). The idea behind this is that the whole collaborative network manifests a joint work, and to have the business up and running, all actors must have enough trust in their partners, otherwise the whole network will collapse because a chain is no stronger than its weakest link.

We also include the trustworthiness of those who express their opinion about a partner in the trust assessment process, and this allows us to achieve a fixed point in the recursion, which then expresses the mutual trust among all partners in the network. 


\subsection{Trust Ontology for Business collaboration Settings}

There are currently some ontologies of business collaboration that facilitate automated collaboration, such as $e^{3}$ value, REA [77, 78], and BMO [79]. However, these ontologies model the situation that all business actors can be trusted. This is not true in practice. To realize automated business collaboration, trust needs to be added to the business ontology. Here, we extend the $e^{3}$ value ontology with the concept of trust and show how this can be used to reason about trust on actors in a business network. We take a minimal approach, i.e. rather than adding all the nuances of the concept of trust, we provide the minimal extension that allows an actor to reason about trusting other actors in a useful way.

\subsubsection{Trust Ontologies}

An ontology is defined as "a specification of a conceptualization." [80]. It specifies the concepts and the relation between the concepts of a specific domain, and they play an important role in knowledge sharing in the specific domain.

In [81], Akkermans and Gordijn introduce the $e^{3}$ value ontology and discuss about the necessity of ontologies for scientific research. Baida et al. [82] developed a multi-actor business model for eservice bundles by ontology-based analysis of e-service bundles in networked enterprises. However their model represent an ideal situation that lacks trust-related issues.

Andersson et al. [83] represented a reference ontology for business models based on three business ontologies - the REA, $e^{3}$ value, and BMO. The core concepts in the REA ontology are Resource, Event, and Actor and it claims that every business transaction can be described as an event in which two actors exchange resources. The Business Model Ontology (BMO) aims at providing an ontology that enables us to describe the business model of an enterprise accurately and in detail, by considering a single enterprise and its environment where the enterprise faces a particular customer's demand. Surprisingly none of these ontologies consider trust-related concepts.

Chang et al. [84] presented the ontological representation of agent trust, service trust, and product trust in e-service environments. The work presented here is similar to the general service/product ontology of Chang. The main difference is that Chang et al do not look at service and product provision necessarily from a business point of view, and consequently they do not include financial risks in their ontologies. They also do not 
discuss the source of trust and the way in which trust develops. Schmidt et al. [85] also proposed a number of ontologies to formalize and facilitate autonomous interactions between intelligent agents in centralized and decentralized e-business environments, however they also do not consider the financial perspective and consequences of trust in the business collaborations.

Haung and Fox [86] try to formalize the semantics of trust and study the transitivity of trust. With formal semantics, they identify two types of trust - trust in belief and trust in performance, and formally prove the transitivity of trust in the former and introduce some conditions under which trust relations of the second type can be propagated. Viljanen [87] surveys and classifies thirteen computational trust models using nine trust decision input factors, and creates a comprehensive ontology for trust to facilitate interaction between business systems. Later, in subsection 6.3.4, we analyze our proposed business trust ontology against those nine factors.

\subsection{2 $E^{3}$ value Business Ontology}

The $e^{3}$ value ontology/methodology [14] is a tractable and lightweight methodology to explore the innovative e-business ideas, starting from understanding which enterprises and actors are actually involved, to an assessment of profitability for each enterprise.

Figure 2-1, which is taken from [27], depicts the $e^{3}$ value ontology for networked business models.

\subsubsection{Trust Ontology for Business Collaboration Settings}

Here we aim at designing a meta model for trust ontology in business collaborations. To do that, we use Howard Becker's recommendation for designing middle-range theories and hypotheses in scientific research, which is describing case-study conclusions without being allowed to mention the specific case itself anywhere [88]. This forces us to come up with and consider more generally valid formulations. This is a good advice for knowledge acquisition in ontology development.

Our goal is to extend the $e^{3}$ value ontology with the minimal ontology of trust to develop a lightweight yet expressive ontology that enables us to describe business collaborations and usefully reason about trust in a business network. We do not want to express all possible meanings of trust, nor do we want to add to the literature on the meanings of "trust" one more bit of insight. We simply want to extend the $e^{3}$ value ontology to make it more 
realistic in the intended settings, that of business networks.

By analyzing different business interactions in different case studies, and also considering the existing trust ontologies, we identified the major trust-related concepts in business collaborations. Then, by delineating the relations between those concepts, we developed a lightweight ontology which contains the minimal set of trust-related concepts in business settings. The ontology is shown in Figure 6-1. The colored concepts are from the $e^{3}$ value ontology and remaining are the new added concepts. Note that the concept of Value Exchange in this thesis is different from its original definition provided by Gordijn [27]. More details are provided in Section 2.1 and in the footnote in page 17 .

For brevity, we exclude those concepts of the $e^{3}$ value ontology which are not directly related to the new added concepts. According to this ontology a trust relation between two business partners is as follows:

A business actor (Trustor) trusts another business actor (Trustee) with a specific confidence (Confidence value). The confidence value is in the range $[0,1]$ and it is calculated based on (1) the reputation (business profile) of Trustee or (2) direct trust ( past experiences/collaborations between the two actors) or 3) indirect trust (the value of the trust of other business actors in the collaboration with Trustee i.e. collaborative trust). A combination of all these three factors is also allowed.

In fact, Trustor expects Trustee to accomplish a certain action during a specific period of time (Time Slot) with agreed upon quality and conditions. In a business collaboration context, this action consists of transferring a specific value object (Value Exchange) with explicit quality specifications in a specified time slot. There is a risk associated with every trust relation which means in case Trustee does not fulfill the agreement (transferring the value object with agreed upon quality), the Trustor will experience a financial loss. The setting of the relation (the actors involved in the collaboration and the relations between them) is described in the value model of the business collaboration.

The financial loss associated with the trust relation originates from the value object and its monetary value. Strictly speaking the potential financial loss of a trust relation is $(1-T) * V$, where $\mathrm{T}$ is the value of trust (confidence value) and $\mathrm{V}$ is the monetary value of the Trustor's value object. This is the case if the Trustor sends his value object to the Trustee, but the Trustee does not send anything back to Trustor. In this case there is a total loss of one value object for the Trustor and the probability of that case 


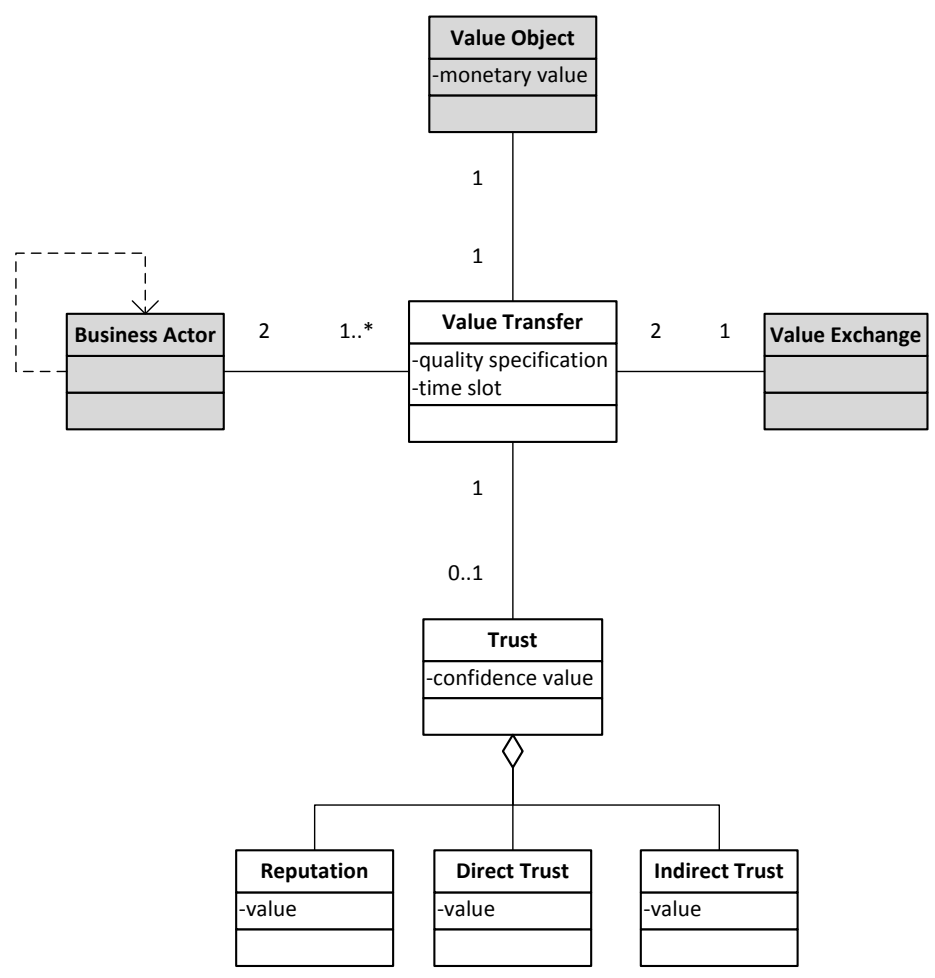

Figure 6-1 Trust ontology in business collaborations settings

is $(1-T)$, where $T$ is the value of trust (confidence value).

This financial loss calculation does not always apply because in some cases a business actor invests a considerable amount of money in the collaboration with another business actor in the hopes of many value exchanges. However, if the other actor misuses the trust early at the collaboration or even at the very beginning, the financial loss of the trustor actor might be much more than the monetary value of the single lost value object.

\subsubsection{Properties of the Trust Ontology}

In this subsection we briefly analyze the presented trust ontology (Figure 6-1) with those nine trust decision input factors enumerated by Viljanen [87]. Trust in our ontology is:

- Identity based: the identity of the actors is known to each other.

- Action aware: Trustor trusts Trustee with a specific action (reciprocity in value exchange).

- Business value-aware: The financial implications of trust in 
terms of potential loss or benefit are the major themes in our ontology.

- Not competence-aware: We have no specific representation of competence of an actor to perform the value exchange as promised. Nevertheless we use a competence in a higher level. We claim that one of the reasons that makes a business actor trust another business actor in a collaborative network is somehow related to competence, because if Trustee does not act trustworthy, the collaboration would fail and consequently Trustee will lose the opportunity to another business actor.

- Not capability-aware: In Viljanen's paper, capability is defined as a form of an access granting token. This is not relevant in our ontology.

- Confidence-aware: We explicitly define the strength of the belief that Trustee exchanges the promised value object with a confidence value.

- Not context-aware: Viljanen defines context as the internal or external status at a particular point of time. In this sense our ontology is not fully context-aware, however we emphasize that the trust relation is valid for a specific period of time regarding a particular action and in a special business collaboration setting, which is modeled in the $e^{3}$ value methodology.

- History-aware: In our ontology past experiences are considered as one of the factors in trust calculation.

- Third-party aware: In our ontology, Trustor uses the opinion of Trustee's direct partners in the trust calculation. The trust calculation method will be explained in Section 6.4.3.

\subsection{Managing Trust within Collaborative Networks}

After introducing a new ontology for business collaborations that includes trust-related concepts in Section 6.3, in this section we introduce an approach for measuring trust in collaborative networks based on collaboration experiences.

\subsubsection{Trust and Value Models}

Having done value modeling, each actor knows with whom it should exchange value objects. So far the model assumes complete trust between partners. But what if two actors, which have direct interaction with each other in the value model, don't trust each other?

Business actors have different options in dealing with the risk 
associated with distrustful partners:

- Accepting the risk. In this case, no change to the value model is needed, and there is no additional investment (to reduce the risk) from the actors, but on the other hand the potential loss is the highest (compared to other options).

- Adding trusted third-parties. A trusted third-party is an actor who both actors trust and sits in the middle and regulates the exchange. The added actor plays a mediator role and it should also be added to the value model $[89,22]$. The mediator accepts the risk of one of the partners not behaving as required by the value model, and asks a payment for this service. In this way the actors substitute the high risk partners with less risky ones and reduce the potential loss.

- Applying controls and safeguards. This option consists of applying appropriate and enough protective and preventive measures in the coordination process and/or IT architecture [40] to lower the risk level to the extent that the remaining risk will be acceptable for collaboration partners. This solution will also affect the value model, but only the computational part; it will increase the cost of value exchange, and reduce profitability estimations.

- Trusted third-parties with controls and safeguards. This option is a combination of the last two options. A thirdparty which sits in the middle can take the responsibility to impose the safeguards and measures. In some cases, even after adding some trusted third-parties, collaboration partners may still want to add specific safeguards and measures. In each case, the added parties and measures would affect the value model and the profitability analysis.

Nonetheless, there is an inevitable trade-off between the profitability and risk. Trusted third-parties and safeguards lower the risk of loss, however the more mediators and/or safeguards sit between the two actors, the more costly would the exchange be, and therefore, the less potential profit there is in the cooperation for both actors.

At the end of value modeling, we come up with a value model in which every actor finds the balance between the risk that a partner behaves opportunistically acceptable compared to the estimated profitability of doing business with that partner. Nevertheless, in a highly volatile collaborative network, some partners may turn out to be less trustworthy than what perceived earlier and, consequently, an actor might want to reconfigure the collaborative network. So, the behavior of the collaboration partners should be 
continuously monitored, and accordingly the trust relations and their values should be continuously updated, so that each partner can keep the balance between profitability and risk throughout the collaboration, and reconfigure the network if and when needed.

If we have a value model of the collaborative network that shows who exchanges which services or products with whom (represented in the $e^{3}$ value graphical part), enriched by the cost of including safeguards in the coordination process and/or IT architecture (in the computational part of $e^{3}$ value model), then the next step is to analyze the remaining risk which each actor should take in order to operationalize the value model.

\subsubsection{Identifying Trust Relationships Between Actors}

To identify the trust relations between actors in a collaborative network, we need to look at the order in which actors exchange value objects with each other. Each specific order implies a certain trust relation. There are three orders in which actors A and B can exchange value objects:

1. Actor A first sends its value object to actor B.

2. Actor B first sends its value object to actor A.

3. Value objects are sent in an interleaved way.

To illustrate this point, let's consider the simple value exchange and its possible realizations in the coordination model shown in Figure 3-15. Figure 3-15(a) shows two actors (A and B) that exchange a pair of value objects $\left(V_{a}\right.$ and $\left.V_{b}\right)$ with each other. Figure 3-15(b) shows a coordination pattern in which actor $B$ first sends its value object $\left(V_{b}\right)$ to actor $A$ and then actor $A$ sends its value object $\left(\mathrm{V}_{\mathrm{a}}\right)$ to actor B. Figure 3-15(c) depicts the other way around.

Figure 3-15(d) depicts a special coordination pattern in which actor $B$ sends part of its value object $\left(V_{b}\right)$, and then actor $A$ sends its value object $\left(V_{a}\right)$ to actor $B$; finally actor $B$ sends the rest of its value object $\left(V_{b}\right)$ to actor $A$. This coordination pattern is feasible only if value object $\left(\mathrm{V}_{\mathrm{b}}\right)$ can be broken into some smaller parts. In general, if value objects are breakable to smaller parts, any combination of value transfers is possible, however for the purpose of our study they all are the same, i.e., in all cases both actors, at some point during the value exchange process, send a value object (or part of a value object) to the other actor in the hope that the other actor acts reciprocally and sends back what is promised.

The three different coordination patterns shown in Figure 3-15 are the only rational possible patterns for coordination messages 
from value exchange order point of view, provided that A initiates the message exchange [7].

In the coordination pattern shown in Figure 3-15(b), first A requests $B$ to send it $V_{b}$ then $B$ sends $V_{b}$ to $A$ and afterwards, in turn, $B$ requests $V_{a}$ from $A$ and finally $A$ sends $V_{a}$ to $B$. Hence, in this case, only actor $B$ needs to trust actor $A$, and not vice versa, because $A$ sends its value object $\left(V_{a}\right)$ only after it receives $V_{b}$. Similarly, in the coordination pattern shown in Figure 3-15(c) only actor A needs to trust actor B. Finally, the coordination pattern shown in Figure 3-15(d) depicts a case in which both actors need to trust each other at some point during the collaboration because each of them send some value to the other one in the hope that the receiver acts reciprocally and fulfils the agreements. All these three different coordination patterns are consistent with the same value model $[7,8]$. In other words, from value point of view they all depict the same situation which is shown in Figure 3-15(a).

Here, for simplicity, we assume that actor A always initiates the message exchange. This will not affect the generality of the discussion because for our trust reasoning, the only thing that matters is the order in which value objects are exchanged. If we drop that assumption, there would be other variation(s) for the coordination patterns shown in Figure 3-15. For example, for the pattern in Figure 3-15(b), there would be another variant in which the messages are in the same order as the current pattern except that the message labeled with 'Request $\mathrm{V}_{\mathrm{a}}$ ' would be the first message. However, in both cases the order of the value transfers is the same (first 'Send $V_{a}$ ' and then 'Send $V_{b}$ '). From value point of view, all different variations of the same pattern are equivalent as long as the exchange order of the value objects is fixed [7].

Therefore, to develop the coordination model we must have the trust relationships between actors. If $\mathrm{B}$ trusts $\mathrm{A}$, the coordination pattern shown in Figure 3-15(b) is the appropriate coordination pattern; and if A trusts $\mathrm{B}$, the coordination pattern shown in Figure 3-15(c) is the appropriate coordination pattern; and finally if $\mathrm{A}$ and $\mathrm{B}$ both trust each other, all three coordination patterns are acceptable.

To design a coordination model based on a value model we need to have the trust relation between each pair of business actors who exchange value objects together in the value model. Having the value model and trust relations between business actors we can develop the coordination model by mapping each value object exchange onto its appropriate coordination pattern. 


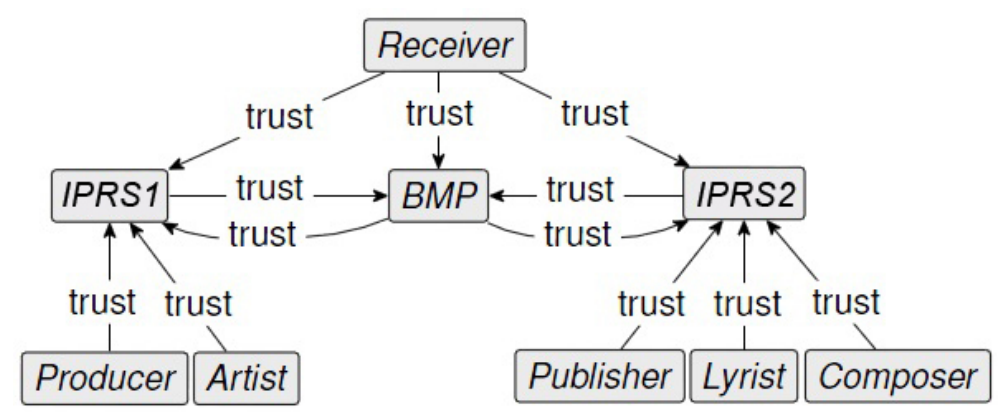

Figure 6-2 Trust model

Suppose that the trust model shown in Figure 6-2 is associated with the value model in Figure $3-16$. In this model if actor $\mathrm{A}$ is connected to actor $\mathrm{B}$ with a directed arrow pointing to $\mathrm{B}$ labeled by 'trust', it means that A trusts $B$ to act reciprocally according to the obligations indicated in the value model.

Actors participating in the value model must agree upon the trust model, however, trust relations may change over time. For example in Figure 6-2, the actor Receiver trusts the actor IPRS1 but not vice versa. Nevertheless, after doing some business, Receiver can build a good reputation for itself and due to that IPRS1 may start to trust Receiver. This will result in an arrow from IPRS1 to Receiver in the trust model. Before this change, the only acceptable coordination message pattern for the value exchange between IPRS1 and Receiver was the pattern shown in Figure 3-15(b) in which A represents IPRS1 and B represents Receiver. After the aforementioned change in the trust model, all the three coordination patterns are acceptable. In this way, a change in the trust relations can lead to a change in the coordination model.

\subsubsection{Measuring Trust}

Assume that for the value exchange in Figure 3-15(a) actors (A and B) agree on the coordination model shown in Figure 3-15(b). This means that $\mathrm{B}$ trusts $\mathrm{A}$ to act reciprocally according to the obligations indicated in the value model. Assume also that B can assign a monetary value to both $\mathrm{V}_{\mathrm{a}}$ and $\mathrm{V}_{\mathrm{b}}$ from its own point of view.

Obviously there is a risk associated with each trust relation and, thus, actor B wants to have an assessment of the amount of possible risk. To do that, B first estimates the maximum possible harm or loss which actor A can do by acting opportunistically (not acting according to the obligations indicated in the value 
model). We assume that the worst case is when $\mathrm{A}$ does not give back the favor to $B$ after receiving $V_{b}$ (A does not send $V_{a}$ to $B$ ), so with respect to a value model $(\mathrm{V})$, we set $\mathrm{V}_{\mathrm{b}}$ as the maximum possible harm which $A$ can impose to $\mathrm{B}\left(\mathrm{H}^{\mathrm{v}}(\mathrm{A} \Rightarrow \mathrm{B})=\mathrm{V}_{\mathrm{b}}\right)$. In some special cases, this is not a true assumption. For example, suppose that B expects A to buy 10 bicycles one by one, and $\mathrm{B}$ has already made some purchases to be able to provide A with 10 bicycles, but A does not pay for the first bicycle after receiving it. This will break the collaboration and the loss, in that case, is obviously more than one bicycle $\left(\mathrm{V}_{\mathrm{b}}\right)$ for $\mathrm{B}$.

Nevertheless, if one side of the coin is loss, the other side is profit, therefore actor $\mathrm{B}$ estimates the possible profit which comes from each successful value exchange $\left(\mathrm{P}^{\mathrm{v}}(\mathrm{B} \Rightarrow \mathrm{A})=\mathrm{V}_{\mathrm{a}}-\mathrm{V}_{\mathrm{b}}\right)$. Now, if $\mathrm{B}$ has a true indication of the probability of A acting opportunistically $\left(\mathrm{R}^{\mathrm{v}}(\mathrm{A} \Rightarrow \mathrm{B})\right)$, it can decide whether to collaborate with $\mathrm{A}$ or not by assessing the following profit and loss equation:

$$
R^{v}(A \Rightarrow B) * H^{v}(A \Rightarrow B)<\left(1-R^{v}(A \Rightarrow B)\right) * P^{v}(B \Rightarrow A)
$$

We define $\mathrm{T}^{\mathrm{v}}(\mathrm{B} \Rightarrow \mathrm{A})=1-\mathrm{R}^{\mathrm{v}}(\mathrm{A} \Rightarrow \mathrm{B})=\alpha, 0 \leq \alpha \leq 1$ as the total trust of $\mathrm{B}$ in $\mathrm{A}$ regarding value model $\mathrm{V}$. Total trust of $\mathrm{B}$ in $\mathrm{A}$ is the possibility that $\mathrm{A}$ behaves as expected. While some trust modeling researchers restrict themselves to binary ratings (either 1 or 0 , to reflect satisfaction or dissatisfaction, such as, e.g. [90],[91]), some others examine ratings in the range [0, 1] (e.g, [92]). The latter is the convention which we adopt in this thesis. $\mathrm{T}^{\mathrm{v}}(\mathrm{B} \Rightarrow \mathrm{A})$ consists of two parts, which we name direct trust and indirect trust of B in A. Here, for simplicity, the Trustee's reputation is considered as part of direct trust.

The direct trust of $\mathrm{B}$ in $\mathrm{A}\left(T_{B \Rightarrow A}^{v}\right)$ is determined by $\mathrm{B}$ based on its empirical knowledge of A established on previous collaborations, A's reputation in the market, etc. Actor B can use different ways to assess the organizational trust of A, which we don't discuss here. There are only a few quantitative measures that express organizational trust. Among the simplest and most popular ones is the employee fluctuation rate, indicating the employee satisfaction and organizational climate. In fact, the members of an organization have the best knowledge about the overall situation of their organization [93].

The indirect trust of $\mathrm{B}$ in $\mathrm{A}$ is a weighted sum of the direct trust of those actors that have a trust relation with $\mathrm{A}$ in the trust 
model (those who should trust A). Hence,

$$
\begin{array}{r}
T^{v}(B \Rightarrow A)=w T_{B \Rightarrow A}^{v}+\sum_{S_{k} \top A, S_{k} \neq B} w_{k} T_{S_{k} \Rightarrow A}^{v} \\
w+\sum_{S_{k} \top A, S_{k} \neq B} w_{k}=1
\end{array}
$$

in which $\mathrm{T}_{\mathrm{B}}^{\mathrm{v}} \Rightarrow \mathrm{A}$ is the direct trust of actor $\mathrm{B}$ in actor $\mathrm{A}$ regarding value model $\mathrm{v}$, and $\mathrm{S}_{\mathrm{k}}$ is an actor who has a trust relation with $\mathrm{A}$ in the trust model $\left(\mathrm{S}_{\mathrm{k}} \top \mathrm{A}\right)$, actor $\mathrm{S}_{\mathrm{k}}$ is different than actor $\mathrm{B}$ and $0 \leq \mathrm{w} \leq 1,0 \leq \mathrm{w}_{\mathrm{k}} \leq 1$ are the weights which actor $\mathrm{B}$ gives to its opinion and the opinion of actor $\mathrm{S}_{\mathrm{k}}$ regarding the trustworthiness of actor A respectively. Each actor assigns different weights to the opinions of its partners or may give the same weight to the opinions of all actors $\left(\mathrm{w}_{\mathrm{k}}=(1-\mathrm{w}) /(\mathrm{n}-1), \mathrm{n}\right.$ is the number of actors who have a trust relation with $\mathrm{A})$.

Because actor $B$ also has a trust relation with actor $A$ in the trust model, we can summarize the above formula as follows:

$$
\begin{array}{r}
T^{v}(B \Rightarrow A)=\sum_{S_{k} \top A} w_{k} T_{S_{k}}^{v} \Rightarrow A \\
\sum_{S_{k} \top A} w_{k}=1
\end{array}
$$

in which $\mathrm{S}_{\mathrm{k}}$ is an actor who has a trust relation with actor $\mathrm{A}$ in the trust model $\left(S_{k} \rightarrow A\right)$ including actor B itself, and $0 \leq w_{k} \leq 1$ is the weight which actor $B$ gives to the opinion of $S_{k}$ regarding the trustworthiness of $\mathrm{A}$.

To illustrate this, the trust computations associated with the trust model in Figure 6-2 are shown in Table 6-1. In this table, the trust of Receiver in IPRS1 ( $\left.\mathrm{T}^{\mathrm{v}}(\operatorname{Rec} \Rightarrow \mathrm{IP} 1)\right)$ is shown in front of the row labeled by Receiver and under the column labeled by the IPRS1. Under the column of IPRS1 the trust value of those actors which should trust IPRS1 can be seen, namely the Receiver, BMP, Producer and Artist. Therefore, according to Formula 6.3 , the new value of $\mathrm{T}^{\mathrm{v}}(\mathrm{Rec} \Rightarrow \mathrm{IP} 1)$ is a weighted sum of all the trust values in the same column, namely $\mathrm{T}^{\mathrm{v}}$ (Art $\left.\Rightarrow \mathrm{IP} 1\right)$, $\mathrm{T}^{\mathrm{v}}(\mathrm{BMP} \Rightarrow \mathrm{IP} 1), \mathrm{T}^{\mathrm{v}}($ Pro $\Rightarrow$ IP 1$)$ and $\mathrm{T}^{\mathrm{v}}(\mathrm{Rec} \Rightarrow \mathrm{IP} 1)$.

For bootstrapping, we can initialize the trust values based on reputation, previous experiences, etc. When actor $\mathrm{B}$ wants to send a value to actor $A$ it computes the trust $\left(\mathrm{T}^{\mathrm{v}}(\mathrm{B} \Rightarrow \mathrm{A})\right)$ according to Formula 6.3 and then it sends the value only if the profit and loss inequality (Formula (6.1)) is satisfied.

Following the transaction, actor $\mathrm{B}$ reevaluates the direct trust value in $\mathrm{A}$, after the true value of delivered good or service is 
Table 6-1 Trust computations for trust model in Figure 6-2

\begin{tabular}{|c|c|c|c|}
\hline & BMP & IPRS2 (IP2) & IPRS1 (IP1) \\
\hline Artist (Art) & & & $\mathrm{T}^{\mathrm{v}}(\mathrm{Art} \Rightarrow \mathrm{IP} 1)$ \\
\hline BMP & & $\mathrm{T}^{\mathrm{v}}(\mathrm{BMP} \Rightarrow \mathrm{IP} 2)$ & $\mathrm{T}^{\mathrm{v}}(\mathrm{BMP} \Rightarrow \mathrm{IP} 1)$ \\
\hline IPRS 2（IP 2) & $\mathrm{T}^{\mathrm{v}}(\mathrm{IP} 2 \Rightarrow \mathrm{BMP})$ & & \\
\hline Composer (Com) & & $\mathrm{T}^{\mathrm{v}}(\mathrm{Com} \Rightarrow \mathrm{IP} 2)$ & \\
\hline Lyrist (Lyr) & & $\mathrm{T}^{\mathrm{v}}(\mathrm{Lyr} \Rightarrow \mathrm{IP} 2)$ & \\
\hline Producer (Pro) & & & $\mathrm{T}^{\mathrm{v}}($ Pro $\Rightarrow$ IP 1$)$ \\
\hline Publisher (Pub) & & $\mathrm{T}^{\mathrm{v}}(\mathrm{Pub} \Rightarrow \mathrm{IP} 2)$ & \\
\hline Receiver (Rec) & $\mathrm{T}^{\mathrm{v}}(\operatorname{Rec} \Rightarrow \mathrm{BMP})$ & $\mathrm{T}^{\mathrm{v}}(\operatorname{Rec} \Rightarrow \mathrm{IP} 2)$ & $\mathrm{T}^{\mathrm{v}}(\operatorname{Rec} \Rightarrow \mathrm{IP} 1)$ \\
\hline IPRS1 (IP1) & $\mathrm{T}^{\mathrm{v}}(\mathrm{IP} 1 \Rightarrow \mathrm{BMP})$ & & \\
\hline
\end{tabular}

evaluated and compared to the expected value promised by actor A. In this way, the behavior of the actor A impacts the new value and this process goes on and on.

The proposed trust value is a function of the opinions of all actors regarding a particular actor, and this value, in fact, reflects the expected behavior of the actor in the collaboration. If an actor performs an action in an exchange which is not according to the promised obligations, this not only affects the trust of its direct partner in that particular exchange but it also affects the trust of other partners in relation with him. Whenever the trust of actor B in actor A drops below a particular level, actor B doesn't trust actor A anymore and the whole collaborative network will collapse. At this point we can resolve the trust problem either by changing the trust relationships between the actors or by designing a new collaborative network from the scratch.

\subsubsection{Simulation}

To illustrate how the formula works in practice we run a simulation for the trust model shown in Figure 6-2. The initial quantified trust relations and the weights of the opinions are shown in Tables 6-2 and 6-3 respectively. For the sake of space we only write the first three letters of the actors' names in these tables. Each number in the trust table represents the amount of trust relation from the actor indicated by the row entry to the actor indicated by the column entry. A number in the table of weights indicates the weight of the opinion of the actor indicated by the column entry for the actor indicated by the row entry.

It is worth mentioning that for each formula, the sum of the weights should be 1 . Considering the following two equations which are used to recalculate the new amount of the trust of the Receiver in BMP and IPRS1: 
$\mathrm{T}^{\mathrm{v}}($ Receiver $\Rightarrow \mathrm{BMP})=$

$\mathrm{W}\left[\right.$ Receiver, IPRS2] $* \mathrm{~T}^{\mathrm{v}}(\mathrm{IPRS} 2 \Rightarrow \mathrm{BMP})+$

$\mathrm{W}[$ Receiver, Receiver $] * \mathrm{~T}^{\mathrm{v}}($ Receiver $\Rightarrow \mathrm{BMP})+$

$\mathrm{W}\left[\right.$ Receiver, IPRS1] $* \mathrm{~T}^{\mathrm{v}}(\mathrm{IPRS} 1 \Rightarrow \mathrm{BMP})$

and

$\mathrm{T}^{\mathrm{v}}($ Receiver $\Rightarrow$ IPRS1) $=$

$\mathrm{W}[$ Receiver, Artist $] * \mathrm{~T}^{\mathrm{v}}($ Artist $\Rightarrow$ IPRS1 $)+$

$\mathrm{W}[$ Receiver, $\mathrm{BMP}] * \mathrm{~T}^{\mathrm{v}}(\mathrm{BMP} \Rightarrow \mathrm{IPRS} 1)+$

$\mathrm{W}[$ Receiver, Producer $] * \mathrm{~T}^{\mathrm{v}}($ Producer $\Rightarrow$ IPRS1 $)+$

$\mathrm{W}[$ Receiver, Receiver $] * \mathrm{~T}^{\mathrm{v}}($ Receiver $\Rightarrow$ IPRS1)

So,

$\mathrm{W}[$ Receiver, IPRS2 $]+\mathrm{W}[$ Receiver, Receiver $]+\mathrm{W}[$ Receiver, IPRS1 $]=$ $0.3+0.4+0.3=1$

and

$\mathrm{W}[$ Receiver, Artist $]+\mathrm{W}[$ Receiver, $\mathrm{BMP}]+\mathrm{W}[$ Receiver, Producer $]+$ $\mathrm{W}[$ Receiver, Receiver $]=0.2+0.2+0.2+0.4=1$

Here, the Receiver assigns the same weight to its opinions regarding the trustworthiness of BMP and IPRS1 in both formulas. In a general case, due to the structure of the trust network and the wishes of the actors regarding the weights of the trust opinions, they might use different weights for their opinions in different formulas, preserving the criterion that the sum of all the weights in each formula should be 1 . In that case, we need more than one table to show weights.

Having the initial tables of trust and weights, we can compute new values for the trust relations. Table $6-4$ shows the trust table after one complete transaction. In other words, in a complete transaction, each participating actor before sending its value object to another actor, recomputes the trustworthiness of that actor using Formula 6.3 and then sends the value object only if the profit and loss inequality (Formula (6.1)) holds. We assume here that the transaction completes correctly and all the actors fulfill their promises. We also assume, for simplicity, that the actors assign the computed values as the new numbers of the trust relations. However, in general, they can assign whatever number (between 
Table 6-2 Initial trust table

Table 6-3

Table of weights

\begin{tabular}{|c|c|c|c|c|c|c|c|c|c|}
\hline & Art & BMP & IP2 & Com & Lyr & Pro & Pub & Rec & IP1 \\
\hline Artist (Art) & & & & & & & & & .75 \\
\hline BMP & & & .85 & & & & & & .8 \\
\hline IPRS2 (IP2) & & .9 & & & & & & & \\
\hline Composer (Com) & & & .95 & & & & & & \\
\hline Lyrist (Lyr) & & & .85 & & & & & & \\
\hline Producer (Pro) & & & & & & & & & .85 \\
\hline Publisher (Pub) & & & .75 & & & & & & \\
\hline Receiver (Rec) & & .7 & .8 & & & & & & .9 \\
\hline IPRS1 (IP1) & & .7 & & & & & & & \\
\hline
\end{tabular}

\begin{tabular}{|c|c|c|c|c|c|c|c|c|c|}
\hline & Art & BMP & IP2 & Com & Lyr & Pro & Pub & Rec & IP1 \\
\hline Artist (Art) & 0.7 & 0 & 0 & 0 & 0 & 0.3 & 0 & 0 & 0 \\
\hline BMP & 0.1 & 0.5 & 0 & 0.1 & 0.1 & 0.2 & 0.1 & 0.2 & 0 \\
\hline IPRS2 (IP2) & 0 & 0 & 0.8 & 0 & 0 & 0 & 0 & 0 & 0.2 \\
\hline Composer (Com) & 0 & 0.1 & 0 & 0.4 & 0.2 & 0 & 0.2 & 0.1 & 0 \\
\hline Lyrist (Lyr) & 0 & 0.1 & 0 & 0.1 & 0.6 & 0 & 0.1 & 0.1 & 0 \\
\hline Producer (Pro) & 0.2 & 0.1 & 0 & 0 & 0 & 0.7 & 0 & 0 & 0 \\
\hline Publisher (Pub) & 0 & 0.1 & 0 & 0.2 & 0.2 & 0 & 0.5 & 0 & 0 \\
\hline Receiver (Rec) & 0.2 & 0.2 & 0.3 & 0.1 & 0.1 & 0.2 & 0.2 & 0.4 & 0.3 \\
\hline IPRS1 (IP1) & 0 & 0 & 0.3 & 0 & 0 & 0 & 0 & 0.1 & 0.6 \\
\hline
\end{tabular}

0 and 1) to the trustworthiness of their partners at any point in time.

It can be easily seen that all the non-zero trust numbers in the same column of the trust table converge and finally, in this particular case as shown in Table 6-5, after 19 transactions, they all become the same (up to three decimal points). The basic question now is: "What does this convergence of trust numbers mean?" It simply means that all the actors in contact with one actor will finally get the same opinion regarding the trustworthiness of that actor.

Table 6-4

Trust table after first transaction

\begin{tabular}{|c|c|c|c|c|c|c|c|c|c|}
\hline & Art & BMP & IP2 & Com & Lyr & Pro & Pub & Rec & IP1 \\
\hline Artist (Art) & & & & & & & & & .78 \\
\hline BMP & & & .84 & & & & & & .825 \\
\hline IPRS2 (IP2) & & .86 & & & & & & & \\
\hline Composer (Com) & & & .864 & & & & & & \\
\hline Lyrist (Lyr) & & & .845 & & & & & & \\
\hline Producer (Pro) & & & & & & & & & .825 \\
\hline Publisher (Pub) & & & .82 & & & & & & \\
\hline Receiver (Rec) & & .76 & .82 & & & & & & .84 \\
\hline IPRS1 (IP1) & & .76 & & & & & & & \\
\hline
\end{tabular}


Table 6-5 Trust table after 19 transactions

\begin{tabular}{|c|c|c|c|c|c|c|c|c|c|}
\hline & Art & BMP & IP2 & Com & Lyr & Pro & Pub & Rec & IP1 \\
\hline Artist (Art) & & & & & & & & & .808 \\
\hline BMP & & & .838 & & & & & & .808 \\
\hline IPRS2 (IP2) & & .820 & & & & & & & \\
\hline Composer (Com) & & & .838 & & & & & & \\
\hline Lyrist (Lyr) & & & .838 & & & & & & \\
\hline Producer (Pro) & & & & & & & & & .808 \\
\hline Publisher (Pub) & & & .838 & & & & & & \\
\hline Receiver (Rec) & & .820 & .838 & & & & & & .808 \\
\hline IPRS1 (IP1) & & .820 & & & & & & & \\
\hline
\end{tabular}

\subsection{Discussion}

We adopted a simple definition of trust, as the probability that one party assigns to the cooperative behavior of another party. This view of trust accordingly asserts that the expectation by one party of the probability of such cooperative behavior by another party will be based both on "hard" data such as the existence of contracts, etc. and on "soft" data, such as one's faith in another party's morality. Hence, we introduced a formula to evaluate the trustworthiness of an actor by an inquiry into public opinion regarding that actor.

Zhang [94] pointed out two distinct problems in computing trust based on polling:

- Ballot stuffing: when an advisor provides an unfairly high rating, and

- Bad mouthing: when the advisor rates unfairly low (for example, in order to drive a business actor out of the marketplace). Our proposed formula (6.3) does not suffer from "ballot stuffing" because, for computing the trustworthiness of a particular actor, it uses the opinions of all the actors in relation with that actor, and if one of those actors provide an unfairly high rate it does not affect the overall rating too much. Besides, actors assigns a particular weight to the opinion of each actor and this can decrease the side effects even further.

The proposed formula is not also affected by "bad mouthing" either because of the same aforementioned reasons and also because in principle a collaborative network is a joint work and manifests a collaboration of a group of business actors which willingly team up in order to provide a good or service to the customer in order to make some value (money) for themselves. The "bad mouthing" problem occurs mostly where there is a bilateral relation between the actors (e.g. independent buyers and sellers). Therefore, it is unlikely that stakeholders rate anybody unfairly 
low because if they do, the cooperation may terminate and that is to the detriment of all actors including the actor who provides an unfair low rate. Nevertheless, the proposed formula adhere to the principle that trust is difficult to build up but easy to lose.

Based on this system, a business actor can decide with whom to do business by looking up a partner's feedback. A potential partner that has predominantly positive feedback will do more business than a user that has mostly negative feedback. The above method has the problem of "reputation squeeze" which is caused because all the products are treated equally without taking the value of the exchanged product into consideration. For example, it treats the feedback given to the exchanges of two products of values $20 \$$ and $2000 \$$ the same way. Nevertheless, this can be addressed by taking the value (in money) of the product associated with each opinion into account when we assign a weight to that opinion, or introduce another coefficient based on the value (money) associated with each opinion.

Here we only use the last opinions of actors, but we can use more than one opinion for each actor (e.g. 3 last opinions) and use an aging function to weigh up the most recent ones over the older ones. The larger the number of ratings used in the evaluation of reputation values, the higher the long-term prediction accuracy of the mechanism. However, since the reputation values are associated with humans, and humans change their behavior over time it is desirable to disregard extremely old ratings. Thus, we ensure that the predicted reputation values are closer to the current behavior of the individuals rather than their overall performance. Another possible extension, which we can think of, is breaking the trust value into some components based on the critical attributes of the goods or services (e.g. delay, quality, etc.) and compute the trustworthiness of the actors regarding each of these attributes separately. The critical attributes mainly revolve around the extent of customer satisfaction, the quality of the product when received as compared to that promised by the vendor, etc. These critical attributes vary according to the product in question. Some examples of the critical attributes are delay in delivery, the quality promised as opposed to the obtained quality, expected price as opposed to price bought, etc. An attribute may be of different importance to different actors. However, computing the trustworthiness of the actors regarding different critical attributes separately is feasible only when there is a consensus on the set of critical attributes. 


\subsection{Related Work}

Trust is a bidirectional relationship between a trustor and one or more trustees, but not inherently symmetric. If the trustor trusts a particular trustee, an equal trust in the opposite direction does not automatically follow. Furthermore, overlapping trust relationships do not necessarily extend to their transitive closure. If an entity trusts another who in turn has a trust relationship with a particular entity, it is not automatically implied that the first entity also trusts that particular entity [93]. Trust is a complex sociological phenomenon and it has been used with varying meanings across publications regarding trust management in cooperations. For a comprehensive overview of the different facets of trust we recommend related surveys from [95, 96, 97]. Our definition of trust relates to $[97,98,93]$. In the process of making a business collaborative network, the strongest influence of trust becomes apparent in the identification phase, entailing the selection of suitable partners for defined business roles. Since time to market and thus speed is essential, partner selection has to take previously unknown partners into account besides already well known ones [99].

Trust has a strong impact on the performance of business collaborations. Dyer and Chu [100] indicated, with empirical evidence, that trust reduces transaction costs and is correlated with increased information sharing in supplier-buyer relationships and leads to better efficiency. Hwang and Burgers [101] enumerated seven properties of trust using an analytical model and pointed out that trust supports cooperation through easing two different types of risks: the risk of being victimized and the risk of losing a trustworthy partner.

In a similar work to ours, Gordijn and Wieringa [89, 22] use the letter of credit, which is an agreement that the bank of the buyer will arrange the payment for the seller as soon as the seller can prove to the bank that he shipped the goods, for dissolving the distrust between a seller and a buyer. The seller proves the shipment by presenting the Bill of Lading to the bank of the seller. In this way they solve the deadlock situation in which on the one hand the seller does not want to ship the goods onto the carrier's vessel (and thereby lose control over them) without first receiving payment from the buyer. On the other hand the buyer does not want to pay the seller (and thereby lose control over the money) before the goods have been shipped. However, in the new situation the seller and the buyer should trust their banks, which 
is obviously less risky than trusting an overseas partner. In fact they substitute the high risk partners with less risky ones. As we already mentioned, our method comes after this phase.

Taking a high level view of our approach, it addresses the choreography problem in the context of inter-organizational business processes. It starts with designing a value model, which shows the agreements and exchanges between the actor in a global level and makes a shared understanding between the business partners, and then designs the choreography model by identifying the trust relations between the participating business partners. The choreography model describes the flow of interactions between the participating business partners that interlink their individual processes in order to satisfy the needs of a customer. In this view, our work is comparable to the work of Hofreiter and Huemer [28] in which they presented a model-driven approach to inter-organizational business processes by extending the UN/CEFACT modeling methodology (UMM) [29]. In fact, these two works are complementary because our emphasis is on the first steps, in which we develop a value model and then a choreography model based on that, however the emphasis of their work is more on the transformation of the chorography model to the business process execution language (WS-BPEL).

\subsection{Conclusions}

In this chapter we first specified how trust is viewed in this thesis, based on contributions reported in literature, and then extended the $e^{3}$ value business ontology with trust-related concepts. Later on we proposed a formula to measure the trustworthiness of an actor in a collaborative network, based on the opinion of the direct partners of that actor. In Chapter 9, we apply the method introduced here on another business case. We enumerated different aspects and properties associated with trust in our special case of interest which drives us toward future research.

There is an indispensable part in each business collaboration which is monitoring. It basically records the performance of the actors participating in the collaboration. Monitoring can be used to determine trust values in Formula 6.1, e.g. by detecting noncompliance or deviation from agreed upon properties of an exchanged product or service. Each actor is interested in particular properties of its partners, which should be already specified in mutual business contracts. Identifying the most suitable and 
appropriate characteristics for each product or service in a collaboration, and monitoring them in a way which facilitates an integrated trust modeling is of high value to business. There is also an natural trade-off between trust and risk which deserves more scrutiny in future work. 


\section{Managing Trust in}

\section{Collaborative Networks using Game Theory*}

“... scientific research is compounded of ... empirical procedures, general speculative ideas, and mathematical or abstract reasoning."

— James Bryant Conant (1893-1978) U. S. Chemist and Educator.

In this chapter we discuss the role of trust in a special type of value exchange which we call "Unobservable value exchange". By modeling those special value exchanges as a game and using the concepts and techniques of game theory, we propose a method to calculate the financial impact of trust on this type of value exchange. Then, based on the result of the game, we refine the profitability analysis for business actors participating in the collaborative network.

This chapter addresses the first sub-problem of the third research problem (sub-problem P3.1, Section 1.3). To address that research problem we develop a method to assess the profitability and sustainability of some special collaboration settings using game theory concepts and techniques, and then we refine the initial profitability analysis of the collaborative network.

The chapter starts by an introduction in Section 7.1. Next, Section 7.2 discusses about two types of value exchanges and enu-

${ }^{*}$ This chapter is based on the following paper:

Hassan Fatemi, Marten van Sinderen, and Roel J. Wieringa. Managing trust in business webs using game theory. In The Proceedings of The International Workshop on Networked Virtual Enterprises (NetVE 2012). IEEE Computer Society. Fukuka, Japan. [102] 
merate their similarities and differences. After that, we analyze the unobservable value exchanges by modeling them as an inspection game in Section 7.3. Section 7.4 provides a discussion about the proposed method and finally, the chapter ends with a conclusion in Section 7.5.

\subsection{Introduction}

In Chapter 6, we studied the role of trust in collaborative networks and proposed a method to measure the trustworthiness of business actors in the collaboration. The assumption there was that those business actors who exchange value objects with each other in the collaboration, reassess the trustworthiness of their partners at the end of each exchange by comparing the actual value objects which they have received from their partners with what had been promised according to the value model. This continuous trustworthiness assessment plays a fundamental role in our approach for managing and measuring trust in collaborative networks, proposed in Chapter 6. The continuous trustworthiness assessment is feasible only when value exchanges are observable. In other words, business actors know when value exchanges happen and also at the end of each exchange they receive something which they can use to compare with what they were expecting.

The method proposed in Chapter 6 is suitable for those situations in which value exchanges are straightforward and observable to both actors participating in an exchange e.g. the collaboration between a buyer and a webshop that sells PCs. Each time a buyer buys something from the webshop, at the end of the buying process both actors of the collaboration (webshop and buyer) can tell us how satisfied they are with the collaboration, and if the other party has acted according to the promises and agreements or not. However not all collaborations are of this nature.

There are some special value exchanges which we call "Not $o b$ servable value exchange" and, as their name suggest, they are not observable to both business actors. In fact, they are not observable to one of the business actors in the collaboration, and that business actor needs to trust the other business actor and collaborate with it in the hope that it will not act opportunistically. If there is a "Not observable value exchange" between two business actors, during the collaboration, one of the business actors (i.e. the one who trusts the other one) does not know when the value exchanges happen and if they happen or not at all. In fact, this 
special type of value exchanges are not observable without cost, as opposed to normal value exchanges which are observable to both business actors without any cost or at least with a negligible cost.

The trusting actor needs to employ some measures in order to observe the value exchanges and the collaborative behaviour of its partner. Continuous monitoring is one way by which the trustor can follow the behaviour of the trustee, however, in some cases monitoring is not feasible because it is either technically impossible or too costly. In these cases, one way to go out of the dilemma is to run random inspections. Inspections cost money and therefore the cost of inspections must be taken into account for profitability analysis.

We use an example to clarify the above discussion. A well known example as a "Not observable value exchange" is the collaboration between passengers and public transportation. In some countries, such as the Netherlands, travelers/passengers are not checked for a valid ticket upon entering a train. Instead some agents run an inspection and check, every now and then, to see if everyone inside the train holds a valid ticket or not and if they catch someone without a valid ticket this person is penalised (€ $€ 5$ plus the price of the ticket).

In the collaboration between passengers and the trains in the Netherlands, train operators do not exactly know when a passenger uses the train if they do not run any inspection. This collaboration is totally based on trust, and train operators just hope that everyone who uses the trains buys a valid ticket. There are numerous examples of business collaboration which are similar to this case. In fact, whenever there is a rule or stipulation in a business collaboration which one actor should comply with and the compliance is part of the collaboration (rule compliance), and the other actor can not verify the compliance without inspection (i.e., without costs), the collaboration manifests a similar situation. Consequently, we can model and analyze those business collaborations using the method introduced in this chapter.

In this chapter, we model those business collaborations that include an unobservable value exchange as a game, and using the well established concepts and techniques of game theory we analyze the situation in order to:

1. Find the best strategy for each actor in the game.

2. Refine the profitability analysis of the value model using the result of the game.

3. Enrich the $e^{3}$ value methodology with a new profitability analysis method. 


\subsection{Observable vs. Unobservable Value Exchanges}

The two examples of the previous section, namely the collaboration between the webshop and the customers and the collaboration between the transportation company and the passengers, are shown in $e^{3}$ value models in Figures 7-1 and 7-2, respectively. Comparing the two value models, we do not notice a particular difference between the two collaborations. Apparently in both cases two actors exchange a pair of value objects with each other and both value models abstract from the rest of the details including the way in which the exchanges happen. Value modeling looks at the collaboration from a value point of view, and depicts an ideal case in which business actors are assumed to be trustworthy and act according to the value model.

In the collaboration between the passengers and the transportation company (Figure 7-2), the ideal situation designed as a value model shows only a pair of values being exchanged between two actors namely a passenger and a transportation company. The assumption here is that every passenger buys a valid ticket before using the trains. However, during the execution of the model, without inspection it is not clear when a value exchange happens (i.e. when a passenger uses the train). Hence, despite the similarity of the value models, for these kind of situations, we can not apply the method presented in Chapter 6 for measuring the trustworthiness of the actors, because as mentioned before, the assumption there was that the value exchanges are straightforward and easy to observe.

So far we explained the similarities and differences between two types of value exchanges namely observable and unobservable value exchanges. We also pointed out that for unobservable value exchanges we need to run inspection to find out if the exchange is going as it is described in the value model or not. We also briefly mentioned that inspections cost money and the cost of inspection should be included in the profitability analysis of the value model. Now, the question to be answered is "how often a business actor needs to run an inspection and how these inspections affect the expected payoffs of the business actors?".

If a business actor runs an inspection and it turned out that its partner had acted according to the agreements it would be a waste of money, and if it doesn't run inspections enough, its partner might cheat and therefore it again loses money. In any case, the inspection costs will affect the final expected payoffs of the business actors involved in the value exchange and it should 


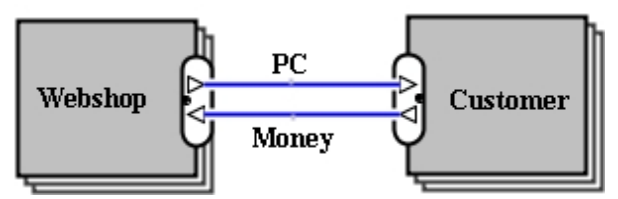

Figure 7-1 The collaboration between webshops and customers

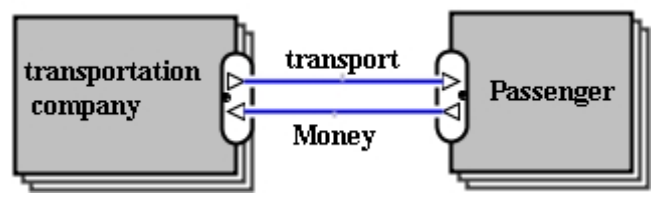

Figure 7-2 The collaboration between trains and passengers

be taken into account. The contribution of this chapter is to provide business actors with a method which helps them find out the best strategy which they should apply in dealing with this special situations and refine the profitability analysis based on the result of the analysis of the game.

\subsection{Inspection Game}

Consider the value exchange labeled with 2 in the value model in Figure 3-16. It shows the exchange of 'Right to make music public' for 'Money'. Receiver agrees to pay IPRS1 for each track which it plays in its public place. It is obvious that this value exchange is an "Unobservable value exchange". The value exchange is not observable at low cost to IPRS1 i.e. without monitoring IPRS1 doesn't know if Receiver acts according to the value model and pays for all the tracks which it plays.

To assess the trustworthiness of Receiver, IPRS1 needs to monitor the behaviour of Receiver continuously during the collaboration. This continuous monitoring might be either by providing Receiver with a temper-proof device to play encoded music records or installing a special device in the place which detects, identifies and reports all the music tracks played in the area. IPRS1 might have full trust in Receiver and consequently rely totally on his reports regarding the played tracks.

The situation between IPRS1 and Receiver corresponds perfectly with inspection in case continuous monitoring is either costly or technically impossible. In fact, if continuous monitoring is not technically possible, the only remaining solution is to employ in- 
spection, and if it is technically possible, there is a trade-off between continuous monitoring and inspection. By comparing the costs and benefits of both solutions, business actors can choose the case which is economically rational, i.e. business actors can select the solution which yields more net profit.

Here, we analyze the solution in which IPRS1 and Receiver are involved in the business collaboration, and IPRS1 trusts Receiver and uses its reports for calculating the amount of money that Receiver should pay for clearing the IPR right of the played music tracks. However, IPRS1 checks the trustworthiness of the behaviour of Receiver by using inspection.

Without inspection Receiver has an incentive to cheat, therefore IPRS1 would like to verify that Receiver is abiding by the agreements, but doing so requires inspections which are costly. If IPRS1 does inspect and catches Receiver cheating, IPRS1 can demand a large penalty payment for the noncompliance. This obviously is a game between IPRS1 and Receiver because 1) IPRS1 and Receiver are both independent actors/players 2) IPRS1 and Receiver each can make decisions that potentially affect the interests of the other player and consequently they can change the outcome/payoff of each other in the game.

We model the collaboration between IPRS1 and Receiver as a game called inspection game. Figure 7-3 shows possible payoffs for such an inspection game. Payoffs are measured in an arbitrary unit and they can be multiplied by a positive number or added with any number, since these two operations keep the relations between the payoffs fixed (having payoffs $P_{1}$ and $P_{2}$ if, for example, $P_{1} \geq P_{2}$ then the relation holds after multiplying both $P_{1}$ and $P_{2}$ by a positive number or adding both of them with any number).

IPRS1 has two strategies or options: it either inspects or does not inspect. In either case, Receiver has two strategies or options too: it either complies or cheats. Hence, there would be four different combinations of strategies. The case in which IPRS1 chooses Don't Inspect, and Receiver chooses Comply yields payoff zero to both IPRS1 and Receiver. In case IPRS1 chooses Don't Inspect and Receiver chooses Cheat, they get payoffs -10 and 10, respectively. If Receiver complies and IPRS1 inspects, Receiver gets payoff 0 , while IPRS1 gets a negative payoff -20 because of the inspection cost. If Receiver cheats, however, inspection results in a heavy penalty (payoff -90 for Receiver) and payoff 70 for IPRS1.

In Figure 7-3, rows represent the strategies of IPRS1 and columns those of Receiver. In general, a player may have more than two 


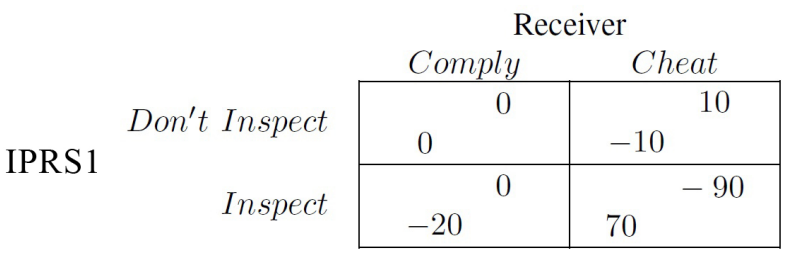

Figure 7-3 Compliance inspection game between IPRS1 and Receiver

strategies. Each strategy combination defines a payoff pair, like $(70,-90)$ for (Inspect,Cheat), which is given in the respective table entry. Each cell of the table shows the payoff to IPRS1 at the (lower) left, and the payoff to Receiver at the (right) top. Note that the quantification is arbitrary.

\subsubsection{Solving the Game}

Like any other game the question here is 'which strategy should each player play?' None of the players can play a strategy for ever because, for example, if IPRS1 always chooses Don't Inspect, the best response for Receiver would be choosing the Cheat strategy, and this yields payoffs -10 and 10 for IPRS1 and Receiver respectively which is to the detriment of IPRS1, and if it always chooses Inspect, the best response for Receiver would be choosing Comply strategy with resulting payoffs -20 and 0 for IPRS1 and Receiver, respectively, again to the detriment of IPRS1. On the other hand, if Receiver settles on Comply, IPRS1 would choose Don't Inspect with resulting payoffs 0 for both players, which is to the detriment of Receiver because it is not to the best interest of Receiver, since if IPRS1 does not inspect Receiver would better off to simply cheat and get payoff 10 instead of 0 . If Receiver settles on Cheat strategy, IPRS1 would prefer Inspect with resulting payoffs -90 and 70 for Receiver and IPRS1, respectively, which is again to the detriment of Receiver. Therefore if, any of the players settles on a deterministic choice the other player can play in his own interest and to the detriment of that player.

There is a technique called max-min strategy, in which each player tries to choose the strategy with the best worst payoff, i.e. the strategy by which the other party can impose less harm. By applying max-min strategy players try to avoid those strategies which might cause big harms. The max-min strategy for IPRS1 is to choose Don't Inspect, since if IPRS1 chooses Don't Inspect strategy then the worst case that might happen is the one in which Receiver chooses Cheat with resulting payoff -10 for IPRS1 
while if IPRS1 chooses Inspect strategy the worst case that might happen is the one in which Receiver chooses Comply and that will result in payoff -20 for IPRS1 (-10 is better than -20$)$.

Analogously, the max-min strategy for Receiver it is to choose Comply since if Receiver chooses the Comply strategy then the worst case that might happen is the one in which IPRS1 chooses Comply, which results in payoff 0 for Receiver, while if Receiver chooses Cheat the worst case is the one in which IPRS1 chooses Inspect and that would result in payoff -90 for Receiver ( 0 is better than -90). Hence, if actors apply max-min strategy, Receiver would choose Comply and IPRS1 would choose Don't Inspect with the resulting payoff 0 for both IPRS1 and Receiver. However, this is not a Nash equilibrium, since Receiver could simply change his strategy and improve his payoff. A pair of strategies, one for each player, forms a Nash equilibrium if even given knowledge of each other's strategies, neither player would have an incentive to switch to an alternate strategy [32]. Therefore, if we treat each player as simply having the two strategies, then there is no Nash equilibrium for this game.

In real life, by playing different strategies randomly, players try to make it difficult if not impossible for their opponents to predict how they play. We will refer to these as mixed strategies and the strategies shown in the payoff table as pure strategies. In a mixed strategy, players rank distributions over payoffs according to their expected values to maximize their expected payoffs. There is a well-established foundation for this in the literature [103, 104, 105].

A mixed strategy for IPRS1 is to inspect only with a certain probability. To find out which probability is the best lets examine two cases. Assume that the probability of inspection is $1 \%$, then, irrespective of that probability, Receiver gets payoff 0 when it complies, and expects payoff

$$
(0.99 * 10)+(0.01 *(-90))=9
$$

when it cheats. Since the payoff in case of cheating (9) is greater than payoff in case of complying (0), Receiver will still cheat, just as in the absence of inspection. If IPRS1 increases the probability of inspection, for example $20 \%$, then the expected payoff for Cheat is

$$
(0.8 * 10)+(0.2 *(-90))=-10
$$

so, Receiver prefers to comply $(0>-10)$. Therefore, if the inspection probability is either too low or too high, Receiver has a unique best response. 
To find out which probabilities IPRS1 should assign to its strategies (Inspect and Don't Inspect) so that Receiver would be indifferent between it strategies (Comply and Cheat), we should solve the following equation:

$$
(10) *(x)+(-90) *(1-x)=0
$$

where $\mathrm{x}$ represents the probability of Don't Inspect strategy. Solving the equation results in $\mathrm{x}=0.90$. Hence the probability of Don't Inspect strategy would be $\% 90$ and consequently the probability of Inspect strategy would be $\% 10$.

Analogously to find out which probabilities Receiver should assign to its strategies (Comply and Cheat) so that IPRS1 would be indifferent between it strategies (Inspect and Don't Inspect), we should solve the following equation:

$$
(-10) *(1-x)=(-20) *(x)+(70) *(1-x)
$$

where $\mathrm{x}$ represents the probability of Comply strategy. Solving the equation results in $\mathrm{x}=0.80$. Hence the probability of Comply strategy would be $\% 80$ and consequently the probability of Cheat strategy would be $\% 20$.

If Receiver was indifferent (i.e. both strategies give it the same payoff), it could choose its strategies randomly. Receiver is indifferent if and only if IPRS1 chooses Inspect with probability 0.1, and Don't Inspect with probability 0.9 because then the expected payoffs for cheat

$$
(0.9 * 10)+(0.1 *(-90))=0
$$

which is the same for Comply. This mixed strategy of IPRS1, makes Receiver indifferent between its strategies.

If IPRS1 was indifferent (i.e. both strategies give it the same payoff), it could choose his strategies randomly. IPRS1 is indifferent if and only if Receiver chooses Comply with probability 0.8 and Cheat with probability 0.2 , because then the expected payoffs for Don't Inspect

$$
(0.8 * 0)+(0.2 *(-10))=-2
$$

and for Inspect

$$
(0.8 *(-20))+(0.2 * 70)=-2
$$

which are the same. These mix strategies (choosing Inspect strategy $10 \%$ of the times and Don't Inspect strategy $90 \%$ of the times 
by IPRS1 and choosing Cheat strategy $20 \%$ of the times and Comply strategy $80 \%$ of the times by Receiver) make the only Nash equilibrium of the game. The resulting expected payoffs are -2 for IPRS1 and 0 for Receiver.

Finding the mixed strategies of two players in a two strategy game using the notion of indifference, when there are no equilibria involving pure strategies, is a general and well-established principle. Each player should randomize between his strategies so that the other player becomes indifferent between its two strategies. This guarantees that neither player's behavior can be exploited by a pure strategy, and those probabilities are best responses to each other. A generalization of this principle applies to games with any finite number of players and any finite number of strategies: Nash proved that every such game has at least one mixed-strategy equilibrium. For more details interested readers are referred to $[106,107]$.

\subsubsection{Adjusting Profitabilities in Value Models}

The resulting expected payoffs of the game ( -2 for IPRS1 and 0 for Receiver) are, in fact, the best payoffs which IPRS1 and Receiver can get out of the situation shown in the payoff table in Figure 73. Note that the standard outcome, defining the reference payoff zero to both IPRS1 and Receiver, in which IPRS1 chooses Don't Inspect and Receiver chooses Comply is, in fact, the ideal case that the value model depicts.

In general, business actors participate in the collaborations because they see profit in value exchanges in the sense that they value what they get higher than what they give. In this particular case, suppose that IPRS1 values what it gets (Money), $€ 10$ more than what it gives (Right). According to the calculations in the previous section, the resulting expected payoff is -2 for IPRS1. Assume also that this is equal to $€-2$, i.e. on average IPRS1 loses $€ 2$ per exchange. Therefore the final value that IPRS1 receives per exchange is $€ 10-€ 2=€ 8$.

Profitability analysis is one part of value modeling and therefore all those "Unobservable value exchanges" should be modeled as a game and analyzed with inspection similar to the case described here. After solving the game and finding the payoffs of the actors in the game, we need to adjust the results, and then business actors will have a more accurate estimates about the performance of the collaboration. The continuous monitoring solutions should be compared with the result of the inspection game and then the solution that yields the best result for business actors 
should be selected.

Now, we propose a refined process for value modeling and profitability analysis for business actors participating in a business collaboration:

1. Build a value model.

2. Determine trust assumptions.

3. Build a game for each value exchange which is not observable without cost for the trusting business actor. The payoff table should be built in cooperation with participating business actors in the value exchange. To do that we should identify all possible options or strategies for each actor, and then assign a payoff value to each actor for the combination of all strategies.

4. Find the average payoff for each business actor in the game.

5. Adjust the values which business actors assume to get from each value exchange with the resulting payoffs of the game.

\subsection{Discussion}

In previous section we saw a game with a mixed equilibrium in which players choose their strategies with certain probabilities. According to the payoffs in Figure 7-3, choosing Inspect strategy by IPRS1 with probability 0.1 makes Receiver indifferent between comply and cheat because the expected payoff for Cheat, namely $(0.9 * 10)+(0.1 *(-90))$, is the same for Receiver as when it chooses Comply. However, this calculation is valid when getting caught incurs only financial loss. In reality, getting caught in business may result in embarrassment and other undesirable consequences. Even though every thing in business boils down to money, however the exact monetary value which we assign to an event such as getting caught is not always straightforward.

Game theory analysis and its results are valid only if monetary values which are assigned to different strategies are valid, and in general, assigning monetary values to all combinations of strategies is the main challenge in analyzing collaborations with game theory. Getting caught is one of the intricate cases. In the compliance game of Section 7.3, the consequences of getting caught for Receiver is assumed to be only the amount of money which it should pay. This assumption might not be true in most of the cases as there are some other issues which Receiver takes into account in deciding how to behave in the collaboration. Shame and the bad reputation which are associated with getting caught also 
play an important role in the decision process for Receiver. Even if we look at the event purely from a financial point of view, there are still some other issues which play role in the decision process. One such issue is the hurdle that getting caught brings with itself. When Receiver gets caught, it needs to go through a process and this process in its easiest and most abstract way takes some time and regardless of the shame some actors might avoid cheating only to prevent those hurdles.

The challenge of assigning monetary values to events and objects is present also in profitability analysis in value modeling. The amount of value which different actors assign to a certain value object depends on their desirability for that value object, and there is 1) no certain way to formulate it and 2) even the actors themselves don't now how to deal with it.

In previous section, we observed that Receiver's best payoff was 0 . That payoff was the result of applying a mixed strategy (choosing Cheat strategy $20 \%$ of the times and Comply strategy $80 \%$ of the times) which is still the same as when Receiver always complies. This seems paradoxical and rises an interesting question. When Receiver could comply and guarantee payoff zero without any risk, why should he gamble? It is because there is an equilibrium only if there is no incentive to choose one strategy over the other [30]. If Receiver always complies, then Don't Inspect would be the best option for IPRS1 and that makes Comply not optimal and, consequently, it would not be an equilibrium.

An interesting aspect of mixed equilibrium is that the probabilities of strategies do not depend on the player's own payoffs, but on the opponent's payoffs [30]. For instance, increasing the penalty for getting caught would not decrease the probability of cheating in the equilibrium. In fact, it decreases the probability of inspection so that Receiver becomes indifferent. This less intuitive property can be explained better in terms of population dynamics in an evolutionary game.

Interpreting the game shown in Figure 7-3 as an evolutionary game demonstrates a non-symmetric interaction between IPRS1 and a group of Receivers. In that case, the probability of a strategy indicates the fraction of interactions in which that strategy is chosen. When the fraction of strategies deviate from the equilibrium probabilities, the strategies that do better get higher probabilities. For example, if IPRS1 chooses Inspect too often, the fraction of cheaters will decrease (they are more afraid of getting caught), which in turn makes Don't Inspect a better strategy. Analogously, if IPRS1 chooses Don't Inspect too often, the frac- 
tion of cheaters will increase (Receivers are less afraid of getting caught). This means that Receivers cheat more often (i.e. the probability of Cheat strategy increases) and this makes Inspect a better strategy for IPRS1. This clearly shows an obvious dynamic interplay between strategies. In this dynamic process, the averages of the fractions of strategies in the long-run, approximate the probabilities of strategies in the equilibrium [30].

\subsection{Conclusions}

In this chapter we identified two types of value exchanges, namely "Observable" and "Unobservable" value exchanges in business collaborations. We also explained that in "Unobservable" value exchanges one of the business actors needs to employ inspection, which cost money, to verify if his partner is abiding by the agreements made in the value model. To find out how often the trusting business actor should run inspections so that the final payoff would be maximum, and how this will affect the expected payoffs, we used the concepts and techniques of the game theory. This is important in business because in some cases, a business actor has no option except trusting his partners in the hope that they would act according to the agreements.

In chapter 9 we provide two real business cases which contain unobservable value exchanges, and then analyze them using the method introduced in this chapter. 
Chapter 7 Managing Trust in Collaborative Networks using Game Theory 
Chapter

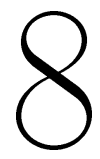

\section{Endurability and Profitability Analysis of Collaborative Networks *}

"Science is a way of thinking much more than it is a body of knowledge."

— Dr. Carl Sagan (1934-1996) American Astronomer, Writer and Scientist.

A collaborative network is a network consisting of a variety of autonomous actors (e.g. enterprises, organizations and people) that collaborate to better achieve common or compatible goals. A collaborative network starts with a contract and then the collaboration partners conduct business as described in the contract. Before engaging in such a collaboration, partners need to reach an agreement regarding their responsibilities in the collaboration, and develop a shared understanding regarding the endurability and the profitability of the collaborative network.

the $e^{3}$ value methodology models business collaborations and provides the business actors participating in the collaboration with a profit analysis. However, the value models and, consequently, the profitability analysis of the $e^{3}$ value are based on the assumption that all business actors participating in the collaboration are trustworthy and they fulfill the promises and agreements. In reality this is not a true assumption and, therefore, we drop this

${ }^{*}$ This chapter is based on the following paper:

Hassan Fatemi, Marten van Sinderen, Roel J. Wieringa, and Ivan S. Razo-Zapata. Endurability and profitability analysis of collaborative networks. In The Proceedings of The 25th Bled eConference. Bled, Slovenia. 2012. [108] 
assumption and enrich the value models of business collaborations with information about trust, in order to provide business actors with more accurate information and estimations regarding the profitability and endurability of the business collaboration.

Here in this chapter, we aim at analyzing the endurability of collaborative networks based on the trust relations between collaboration partners, and also introducing a new approach to do profitability analysis for collaborative networks.

This chapter addresses the second sub-problem of the third research problem (sub-problem P3.2, Section 1.3). To address that research problem we develop a method to assess the endurability and profitability of the collaborative networks, by taking trust into account and, consequently, refining the initial profitability analysis of the collaborative networks.

Section 8.1 discusses the endurability issue in collaborative networks and Section 8.2 addresses the profitability issue in collaborative networks. An illustrative case study is provided in Section 8.3 and then Section 8.4 concludes the chapter.

\subsection{Endurability of Collaborative Networks}

Endurability is a major property of a collaborative network that enables it to continue to function despite undesirable events. For actors participating in a collaborative network, it is vital to have an indication of how durable the collaboration is. Each business collaboration is meant to be operating for a specific period (contract period), and therefore collaboration partners need to know if the collaboration is strong enough to last throughout this contract period.

To analyze the endurability of collaborative networks, we consider two main aspects 1) structural stability 2) execution durability. Structural stability stems from the relations between the partners in the collaboration, while execution durability arises from the way in which partners collaborate with each other.

For structural stability analysis of a collaborative network, we use the initial trust relations between actors, and for execution durability, we exploit the profitability analysis of collaborative networks. We will see that the more initial trust relations there are between the actors and the more profitable collaboration there are, the less vulnerable the collaborative network is, and consequently the more durable the collaboration. 


\subsubsection{Structural Stability of Collaborative Networks}

We use the trust relations between the actors to determine how stable a collaborative network is. To do that, we assume that we are comparing two or more overlapping collaborative networks. We first design a model, which we call general trust model, that represents all actors in all possible collaborative networks and the trust relations between them. The general trust model is a directed graph in which nodes represent business actors participating in the collaborative network, and a directed edge/arrow from node A to node B means that actor A trusts actor B to collaborate with it in a business collaborative network. In other words, the trustor accepts the risks of collaboration with the trustee. However, trust is always with regard to a specific action and here we consider trust with regard to the responsibilities and agreements made in the collaboration contract. When an actor trusts another actor, it means that the trustor trusts the trustee to fulfill the agreements made in the contract and act according to the value model.

We also design a simple model, which we call value exchange model, for each collaborative network. The value exchange model is a simplified version of a value model of $e^{3}$ value. The value exchange model is an undirected graph in which nodes represent the business actors participating in the collaborative network, and an edge between two nodes means that the corresponding business actors of those two nodes are going to be in direct relation with each other in the collaborative network. Being in direct relation implies that they exchange value objects with each other in the value model, and therefore there should be a trust relation between those two actors. We will use the trust model to analyze static stability, and the value exchange model to analyze execution endurability of the collaborative networks.

After designing the general trust model and the value exchange model of each collaborative network, we classify the collaborative networks in three groups, by superimposing the value exchange model associated with each collaborative network on the general trust model as follows:

1. Stable: If there is a mutual trust relation in the general trust model between every two actors that are connected in the value exchange model, then we conclude that the corresponding collaborative network of the value exchange model is structurally stable.

2. Weakly Stable: If there is at least one trust relation in the general trust model between every two actors that are con- 
nected in the value exchange model, and at least one of the trust relations is not mutual, then we conclude that the corresponding collaborative network of the value exchange model is structurally weakly stable.

3. Not Stable: If there is no trust relation in the general trust model between a pair of actors that are connected in the value exchange model, then we conclude that the corresponding collaborative network of the value exchange model is structurally not stable.

Those collaborative networks which fall in the last group (Not Stable) are left out of consideration, since they are not feasible and not realizable, because as Kenneth J. Arrow [63, page 24] pointed out " . . . ethical elements enter in some measure into every contract; without them no market could function. There is an element of trust in every transaction". If there is no trust relation between two actors who are in direct relation with each other, and they need to exchange some value object, we can conclude that that collaboration would not function and therefore it is not realizable. For a collaborative network to be realizable, we need at least one trust relation between every two business actors who are in direct relation with each other in the value exchange model.

Those collaborative networks which fall in the first group (Stable) are better (more enduring) than those that are in the second group (Weakly Stable). In general, the more mutual trust relations between the actors the better because more coordination patterns can be deployed and the realization of the collaboration manifests more flexibilities. We illustrate this point in the following.

Figure 8-1(a) demonstrates a collaboration between two actors, $\mathrm{A}$ and $\mathrm{B}$, in which actor $\mathrm{B}$ provides actor $\mathrm{A}$ with a service for a fee. Assume that at the outset of the collaboration, only A trusts B and B doesn't trust A (see Figure 8-1(b)). Theretofore, actor B agrees to collaborate with actor A only if A pays B before receiving the service each time (see Figure 8-1(c)).

The trust model is dynamic and it can undergo some changes during the course of the collaboration. These changes can be because of the appearance of a new trust relation or disappearance of an existing one, or even the increase or decrease in the trust value of existing trust relations. These changes may imply certain collaboration patterns and therefore actors need to adapt their actions accordingly.

Now, assume that after some time, actor B grows trust in actor $\mathrm{A}$, and therefore a new trust relation is added to the trust model 


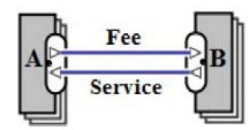

(a) A value exchange

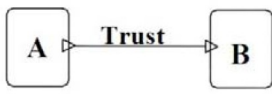

(b) Trust relation between actors

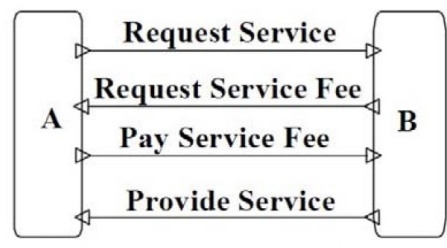

(c) Coordination pattern for paying the fee before receiving the service

Figure 8-1 Possible coordination pattern of a value exchange

(see Figure 8-1(b)). This might change the process model because actor $\mathrm{B}$ might drop the payment condition so that actor $\mathrm{A}$ can pay B after receiving the service. This means that actor A can pay either after, or before or even partly after and partly before receiving the service. This new situation is demonstrated in Figure 8-2. Note that in the coordination pattern shown in Figure 8-1(e), both actors need to trust each other at some point in time during the collaboration, because actor A first pays $20 \%$ of the fee before receiving the service, and then actor $\mathrm{B}$ provides actor $\mathrm{A}$ with the service before receiving the fee of the service completely. These percentages can also change due to the changes in the level of trust that each actor has in the other actor.

Hence, changes in the trust model can implicate some changes in the way that actors collaborate with each other. The interplay between trust relations and coordination patterns demonstrates that the more trust relations there are between business actors, the more coordination patterns can be used in the collaboration model and hence the more flexible the collaboration is.

\subsubsection{Execution Durability of Collaborative Networks}

If there is enough initial trust relations between the collaboration partners, we can implement the business case and put it in action. However, the duration of the collaboration depends on many factors. To name a few: what is the initial trust situation?, how sensitive are the business actors? (do they break the collaboration after noticing that the other partner does not fully respect the promises?), how profitable is the collaboration?, etc. After all, the collaboration is meant to last for a specific period (contract period), so we can rephrase the initial question as: Does the collaboration last throughout the contract period?

The only way to know if the collaboration can last throughout the contract period is to wait until the contract period is over. Nevertheless, there are some indications which can be used in 


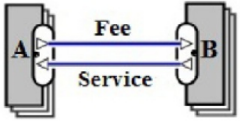

(a) A value exchange.

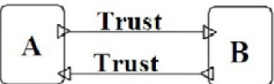

(b) Trust relation between actors

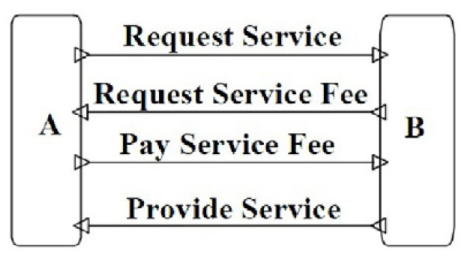

(c) Coordination pattern for paying fee before receiving the service

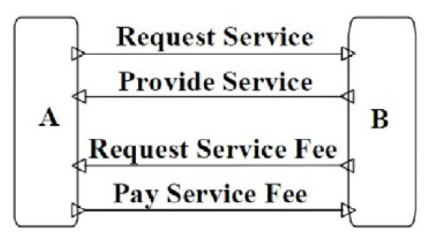

(d) Coordination pattern for paying the fee after receiving the service

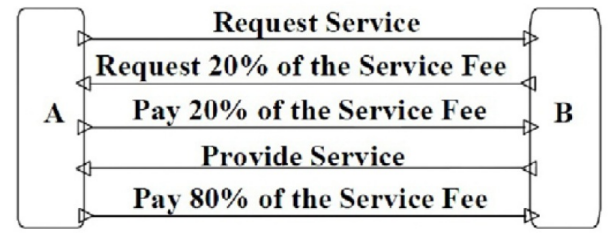

(e) A coordination pattern in which paying the fee and receiving the service are intermingled

Figure 8-2 Possible coordination pattern of a value exchange

the reasoning process. To do that we need to predict the way in which partners will collaborate with each other. If they fulfil their responsibilities in full or at least to a great extent, and if the collaboration is predicted to be profitable enough for all partners, then we can expect that the collaboration will last throughout the contract period. If a partner does not act according to its promises then the continuation of the collaboration depends on the quality of its collaboration performance and also on the sensitivity of its partners.

A collaboration partner might neglect some of the flaws of its partner, or degrade its estimated trust value in the wrong doer partner slightly, while another partner might degrade its estimated trust value severely or even break the collaboration in reaction to the same flaw. The sensitivity of a collaboration partner in breaking a collaboration depends highly on the profitability of the collaboration. In some cases, a collaboration might be still profitable or motivating enough for an actor even if its partner does not act fully according to the agreements, and therefore that actor will still continue with the collaboration though probably with more precautions and preventive measures.

Because of the above reasons it is hard to predict how long the collaboration will last, nevertheless structural stability and equitable profit distribution among the group (i.e. each collaboration partner earns enough profit) are two main criteria which can be considered. In Section 8.1.1, we saw that the more trust 
relations there are between business actors, the more flexible the collaboration is. However, at the same time this indicates that the more durable the collaboration would be. For example, if there is a mutual trust relation between two actors and after some time one of the trust relations disappear (e.g. due to the unsatisfying performance of one of the business actors), there still is a coordination pattern which can be used in the collaboration model and the collaboration can still continue functioning. In contrast, if there is a one-way trust relation between two business actors and that trust relation disappear, then we are in a situation in which none of the two actors which are in direct relation with each other in the collaboration trusts the other anymore, and therefore the collaboration terminates. At this point we should go back to the drawing board and design a whole new collaborative network from the scratch.

In addition to the duality of the trust relations between business actors, the strength of the trust relations also play an important role in the collaboration life time. This is because normally when one business actor does not fully respect the agreements and fails in delivering its undertakings according to the value model, its partners start to decrease their trust in it until they do not trust it anymore. Therefore, the stronger the initial trust relation, the longer it takes till it vanishes and, consequently, the later the collaboration terminates.

Despite the quantity and quality of trust relations between business actors, the final criterion which business actors decide upon to either continue or leave a collaboration is the profitability of the collaboration, since the final goal of participating in any business collaboration for business actors is to earn profit. Therefore, we can simply claim that a collaboration will last as long as the profit each business actor earns still satisfies that actor.

\subsection{Profitability Analysis of Collaborative Networks}

The $e^{3}$ value tool $^{1}$, which we use to model collaborative networks, consists of a graphical part and a computational part. The graphical tool is a diagram editor and the computational tool is a spreadsheet editor with algorithms that can perform Net Present Value (NPV) estimations for the actors in the diagram. To do the profitability analysis of a collaborative network, each partner needs to assign values in terms of money to each value object which it

\footnotetext{
${ }^{1}$ http://e3value.few.vu.nl/
} 
gives and receives. That basically indicates how much each object is worth for each actor. In this way, each actor can measure how much value or money it gets per value exchange, provided that the exchange takes place as described in the value model. Then by estimating the number of value exchanges during the contract period we can tell each actor how much profit it is going to earn out of the collaboration. There are some assumptions in this approach and to better understand the mechanism we need to explain some of these assumptions:

1. Assigning monetary values to all exchanged objects is not always straightforward. If someone is paying $€ 10$ to watch a movie, we generally can assume that watching the movie is worth more than $€ 10$ to that person. However, assigning a specific value to the movie depends on many factors, among others the personal taste of the person regarding that particular movie. In general, one value object can be of different values to different business actors, and that makes assigning fixed monetary value to value objects a challenging task. On the other hand, not only for the receivers of a value object but sometimes for the producers of a value objects it is also challenging to assign it a monetary value.

2. Estimating the number of expected exchanges during the contract period is another challenging issue.

3. The value model describes an ideal situation in which all actors fulfill their responsibilities and act according to the agreements (i.e. actors are assumed to be trustworthy). To have a more realistic estimation of the profitability of a collaborative network, we need to drop this assumption and take into account those factors that can affect the profitability of a collaborative network. It is not easy, if not impossible, to identify all possible risks and the probability and impact associated with each risk.

Dropping each of these assumptions introduce some cost. Here in this chapter, we only discuss the third assumption. Dropping the third assumption would mean monitoring partners' behaviors and inspecting value objects and that impose some cost. In the current state-of-the-art of profitability analysis, which is done based on value models, this cost is not taken into account. To make the profitability analysis results more realistic and reliable, we suggest to assign so called risk cost, to each value exchange, and then adjust the results accordingly.

The amount of surveillance and monitoring that an actor would like to have on the behavior of another actor in the collaboration 
depends on the level of trust between those two actors. Therefore, to estimate the risk cost we first need to quantify the trust relations between the partners. In Chapter 6, we proposed a method for measuring and managing the trust values between the collaboration partners in a collaborative network.

\subsubsection{Computing Profitability Based on Trust}

Consider the value exchange shown in Figure 8-3, which simply means actor A gives value object $V_{a}$ to actor $\mathrm{B}$ and in return actor $\mathrm{B}$ gives value object $V_{b}$ to actor $\mathrm{A}$ and assume that actors $\mathrm{A}$ and B assign the monetary values to value objects $V_{a}$ and $V_{b}$ as shown in Table 8-1. This implies that value object $V_{a}$ costs $€ 10$ for actor $\mathrm{A}$ and it is worth $€ 11$ for actor $\mathrm{B}$ and likewise, value object $V_{b}$ costs $€ 8$ for actor $B$ and it is worth $€ 12$ for actor $A$. This means that stakeholder A earns $€ 2(€ 12-€ 10)$ in each transaction and stakeholder $\mathrm{B}$ earns $€ 3(€ 11-€ 8)$, provided that the exchange takes place as described in the value model and the realization of the value exchange does not impose any extra cost.

Assuming that in a [0-1] scale, the value of the trust of $\mathrm{A}$ in $\mathrm{B}$ is 0.85 and the value of the trust of $\mathrm{B}$ in $\mathrm{A}$ is 0.90 . These numbers can be interpreted in two ways:

1. Probability in each exchange. A is currently $85 \%$ certain that $\mathrm{B}$ will deliver value object $V_{b}$ according to the agreements, and analogously B is $90 \%$ certain that A will deliver value object $V_{a}$ according to the agreements.

2. Probability in the long run. A believes that, in the long run, $85 \%$ of the times $\mathrm{B}$ will deliver value object $V_{b}$ according to the agreements, and similarly $B$ believes that, in the long run, $90 \%$ of the times A will deliver value object $V_{a}$ according to the agreements.

Based on both interpretations, the real profit which A can expect per exchange in this collaboration is $(0.85 * € 2)-(0.15 * € 10)=€ 0.2$. This calculation is based on the reasoning that in each exchange, the probability of a successful exchange is 0.85 and the probability of an unsuccessful exchange is 0.15 . If the exchange goes successfully according to the agreements $A$ earns $€ 2(€ 12-€ 10)$ and in case of an unsuccessful exchange we assume the worst case in which actor A sends his value object $V_{a}$ and receives nothing back in return. In that case, actor A loses its value object completely, which means that A loses $€ 10$. Analogously, in the same line of reasoning, the real profit which $B$ should expect per exchange is $(0.9 * € 3)-(0.10 * € 8)=€ 1.9$.

As another case assume that A does not trust B and only B 


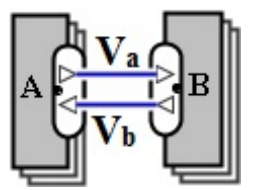

Figure 8-3 A value exchange between two business actors

Table 8-1

Monetary values of $V_{a}$ and $V_{b}$ according to actors $\mathrm{A}$ and $\mathrm{B}$

\begin{tabular}{|c|c|c|}
\hline & $V_{a}$ & $V_{b}$ \\
\hline $\mathrm{A}$ & $€ 10$ & $€ 12$ \\
\hline $\mathrm{B}$ & $€ 11$ & $€ 8$ \\
\hline
\end{tabular}

trusts $\mathrm{A}$, and the value of the trust of B in A is 0.8. First of all, this means that only certain coordination patterns are applicable, and also that $\mathrm{A}$ does not take any risk in this collaboration (i.e. actor A always receives value object $V_{b}$ first, and then sends $\mathrm{B}$ value object $V_{a}$ if it is satisfied with the exchange). Therefore the expected profit of A out of each exchange is $€ 2(€ 12-€ 10)$ and the expected profit of $\mathrm{B}$ is $(0.8 * € 3)-(0.20 * € 8)=€ 0.8$.

In this way all partners should calculate the refined profits, and then decide to engage in the collaboration only if the refined profitability estimations are still acceptable. Using the proposed method for managing trust introduced in Chapter 6, actors can do this calculations at any point in time during the collaboration, and make their decision for continuing the collaboration based on the result of the calculations [5].

What happens in reality is that an actor decides to engage in a collaboration with another actor which actually might be unknown to it. In this case, the actor starts with full trust, and therefore it accepts the risk of collaboration with an unknown actor, which implies that the trusting actor might lose the value object it delivers completely without receiving anything in return. However, the method we proposed in Chapter 7 and [5] to manage the trust relations and the method presented here can be used later in the collaboration.

In Chapter 7 we discussed a special type of value exchange ( $U n$ observable value exchange), and we explained the way we analyze profitability in this type of value exchange. We model these situations as a game and by solving the game we provide the involved actors with the risk cost. This allows the participating actors to adjust their profits accordingly. 


\subsection{An Illustrative Case Study}

We have applied the analysis presented in Section 8.1 on an illustrative case study that is about educational services. The idea is that people are looking for specific jobs that require special skills. Since the required skills for a person (customer) cannot always be provided by a single educational service, it is desirable to find meaningful combinations of services that can jointly provide a valuable solution to the customer, i.e. a Service Value Network (SVN).

We test our solution framework we use a simulation case in which we have harvested the publicly available National Database of Accredited Qualifications (NDAQ), which contains details of Recognized Awarding Organizations and Regulated Qualifications in England, Wales and Northern Ireland that offer different skills through educational services. The final service catalogue is composed of 58 services provided by four service suppliers.

Figures 8-4 and 8-5 depict two possible SVNs that have been composed to cover the need: "As a multimedia creator, How can I improve my skills?". As it can be observed in Figure 8-4, the SVN provides a solution to this need by networking several services. At the top, a service bundle offers the required skills $F C_{1}$ (Digital Image Manipulation), $F C_{2}$ (Photo Image Capture) and $F C_{3}$ (Studio Photography) by combining three educational services. At the bottom, three service enablers support the functionality of the service suppliers.

In this manner the problem at hand is twofold: 1) Provide a set of solutions when a customer needs to acquire some skills. 2) Once the alternative solutions are offered to the customer, to provide a selection criterion so that the customer can choose one option among the set of alternatives.

To deal with the first issue, Ivan et al. have previously presented a framework that (semi)automatically composes alternative SVNs to cope with customer needs [109]. Briefly, whereas a customer expresses his need in terms of the required functionalities (the skills in this context), and then a cluster-based algorithm composes alternative SVNs that can satisfy the given customer need, i.e. alternative networks of educational services [109].

In order to solve the second issue, we propose the analysis described in Sections 8.1 and 8.2 that evaluate the endurability and profitability of a given collaborative network. Consequently, the customer is provided with information that allows him to choose one SVN that not only matches his needs in terms of required 


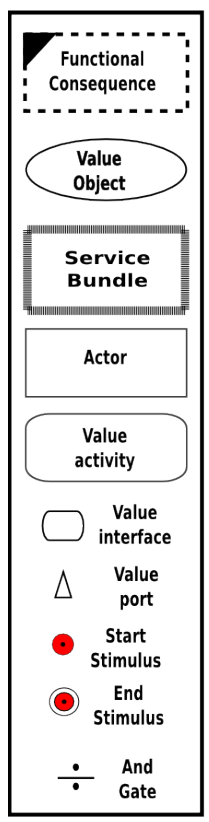

Legend

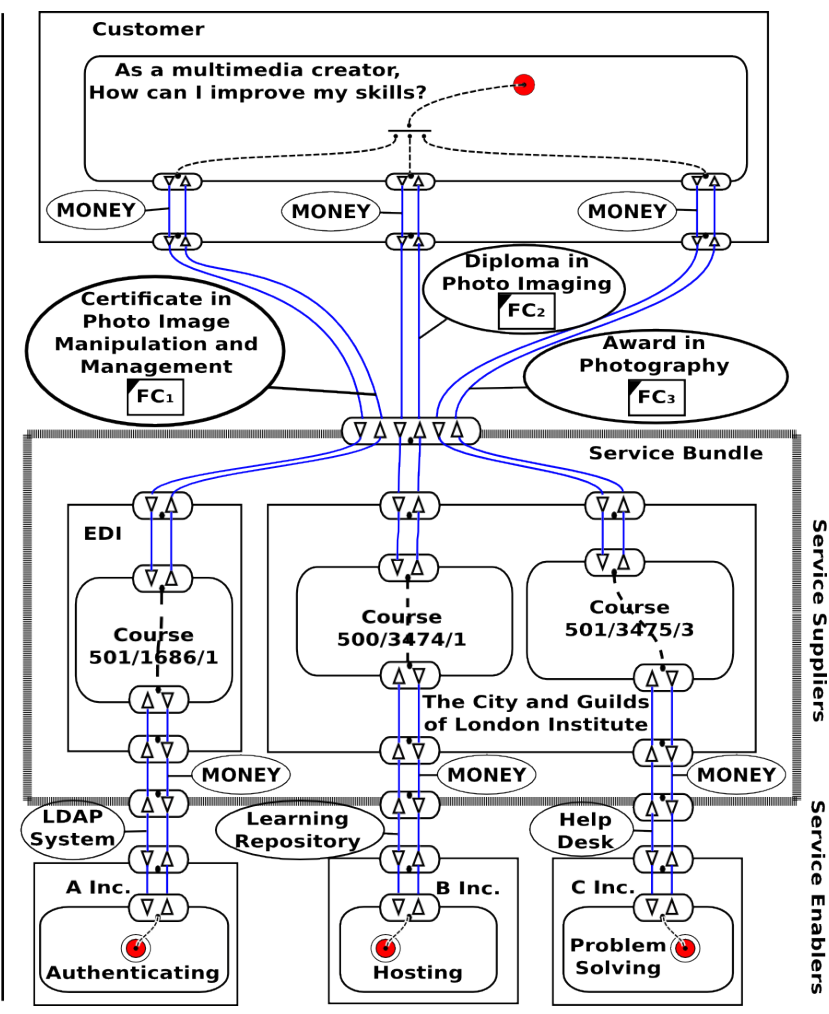

Figure 8-4 A service value network example 


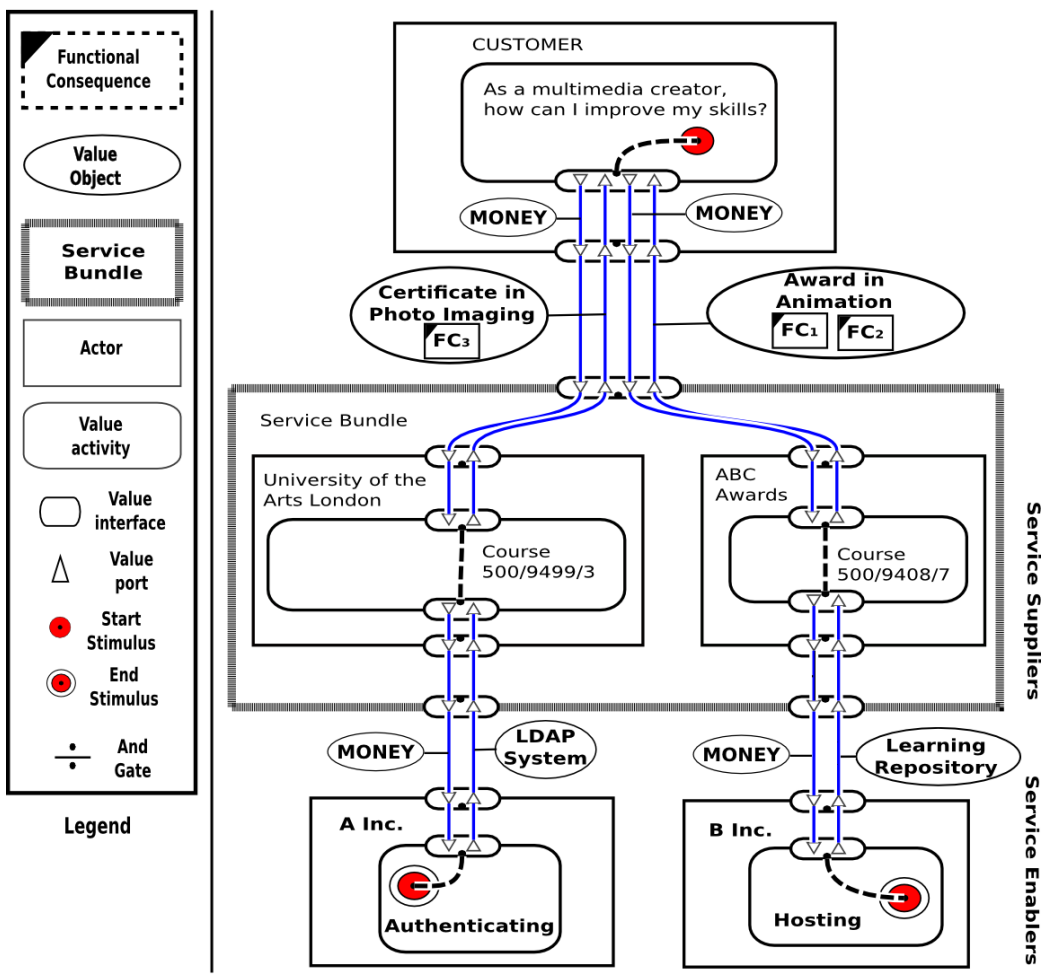

Figure 8-5 A service value network example 


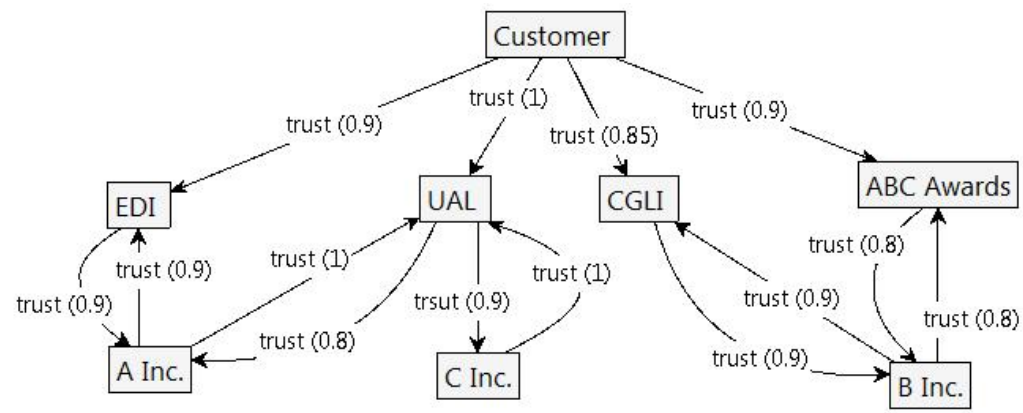

Figure 8-6 General trust model

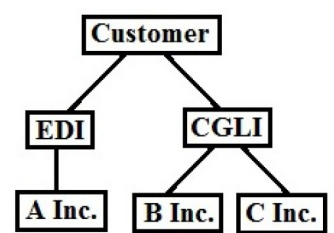

(a): Value exchange model of collaborative network in Figure 4

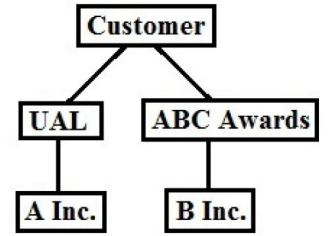

(b): Value exchange model of collaborative network in Figure 5

Figure 8-7 Value exchange models of collaborative networks

skills, but also in terms of economic profitability.

The trust relations and their quantified values are shown in the general trust model (see Figure 8-6). The value exchange models of the two collaborative networks are shown in Figures 8-7. In this models, CGLI and UAL stand for 'the City and Guilds of London Institute' and 'University of the Arts London', respectively.

After superimposing the value exchange models on the general trust model, it turns out that the collaborative network shown in Figure 8-4 is Not Stable, and the collaborative network shown in Figure 8-5 is Weakly Stable. Therefore, we exclude the collaborative network shown in Figure $8-4$ and continue the profitability analysis with the collaborative network shown in Figure 8-5.

To do the profitability analysis, for each value exchange the participating actors should assign monetary values to the value objects they exchange. Here we only explain the value exchange between Customer and UAL (see Figure 8-2). The profitability analysis can be done in the same way for all other value exchanges. As it can be seen in the general trust model, UAL does not trust Customer so it does not take any risk in this collaboration, and therefore, it earns $€(300)$ in this collaboration. However, the trust value of Customer in UAL is 0.95 , and therefore, the expected 
Table 8-2 Monetary values of value objects

\begin{tabular}{|c|c|c|}
\hline & Money & Certificate... \\
\hline Customer & $€ 1000$ & $€ 1200$ \\
\hline UAL & $€ 1000$ & $€ 700$ \\
\hline
\end{tabular}

profit of Customer is $(0.95 * € 200)-(0.05 * € 1000)=€ 140$.

In this case, we can interpret the results as follows. UAL provides Customer with a service (course) and because Customer is unknown to UAL, it does not trust him. Hence, UAL does not take any risks and it charges Customer $€(1000)$ upon registering for the course. On the other hand, based on the reputation of UAL and/or Customer's investigation, Customer believes that with probability of $95 \%$, UAL provides the service (course) as he expects.

\subsection{Conclusions}

In this chapter we analyzed the collaborative networks from endurability and profitability points of view based on the trust relations between the collaboration partners. Providing the partners with value models supplemented by extra information regarding the endurability and profitability of the collaboration enables them to decide on those collaborations which are more durable and profitable. Since value models depict the required exchanges to achieve a business goal, when they are enriched with information about trust, endurability and profitability, they can provide more insights to specify service level agreements among the participants within the network.

In this chapter we addressed the third research problem (problem P3, Section 1.3) which was about developing a method to assess the endurability and profitability of some collaborative networks by taking trust into account. Quantification of the trust relations in a business collaboration is the main limitation of the method introduced here. In some cases, valuation of value objects in terms of money, can also be challenging.

Taking appropriate monitoring and preventive measures in the value exchange process can affect the level of trust which collaboration actors have in each other. For example, an actor that does not trust another actor may consider implementing appropriate safeguards in order to increase the trust (decrease the risk). 

Collaborative Networks 


\section{Validation}

"In science the credit goes to the man who convinces the world, not the man to whom the idea first occurs."

— Sir Francis Darwin (1848 - 1925), Eugenics Review, April 1914

In this chapter we provide additional validation for the methods and approaches introduced in this thesis regarding the issue of trust in business collaborations by means of some simulation cases. Section 9.1 provides an introduction to the chapter, Sections 9.2 and 9.3 discuss two real case business collaborations and analyze them using the method introduced in Chapter 7 . At the end, Section 9.4 discusses another business collaboration case and applies the method introduced in Chapter 6 to this case.

\subsection{Introduction}

In her $\mathrm{PhD}$ thesis, Kartseva [40] developed a methodology that supports human analysts in designing controls against opportunistic behaviour in networks of organizations. She introduced the $e^{3}$ control approach that provides guidelines and conceptual modeling tools to design controls in networks. To do that, she extended the $e^{3}$ value business ontology (see Figure 2-1) to specifically include the concepts required to model the effects of opportunistic behaviour on value transfers.

Having the value model of a collaborative network, she analyzes the network and its value exchanges to identify all those scenarios in which an actor does not act reciprocally, and then adds appropriate control patterns to the value model in order to reduce the risk of opportunistic behaviours. By introducing $s u b$ ideal value objects and sub-ideal value transfers she models the 
sub-ideal behaviours resulting from the incorrect exchange of a value object or the exchange of an inappropriate value object.

Despite its generality, expressiveness and comprehensiveness, there are also some drawbacks in $e^{3}$ control approach:

- In most of cases, the $e^{3}$ control approach substitutes a risk with another one without specifying the tradeoff between these two. In fact, reducing the risk is the only solution for dealing with any risky situation in business, because after all the business actors need to trust someone or something. However, a potential solution for a risky situation in a business collaboration needs to be analyzed financially before the solution is introduced, and the business actors must be provided with the costs and benefits of the proposed solution.

When proposing a control, the $e^{3}$ control methodology does not consider the cost of the control, and sometimes the applicability or feasibility of the control is overlooked. If a control is to reduce the risk, a comparison between the situations with and without the control should be provided, so that each actor would be able to select the situation which it prefers. In some cases, the cost of the control may wash out all the profit of the collaboration for an actor and consequently that control would not be appropriate. The approach lacks such analysis.

- The $e^{3}$ control methodology introduces many concepts and analyzing a case with the methodology makes it quite complicated. In some cases the $e^{3}$ control models contain too many details that make them hard to understand even for the domain experts.

The $e^{3}$ control methodology provides control patterns, which are defined as descriptions of generic and re-usable control mechanisms for a recurring control problem. Nevertheless, the application of a control mechanism differs from case to case, and the approach does not provide detailed information regarding the feasibility (neither technically nor financially) of the implementation of the control mechanism. For example, one control mechanism is 'screening', which can have many different forms, each with a specific cost and impact. The methodology deals with the control in an abstract way, and it does not discuss the application details and the financial impacts of the control, e.g. screening on the specific case at hand.

In the next section we demonstrate the positive and negative points in Kartseva's approach by analyzing two of the case studies which she has studied in her thesis. We apply our approach 
on those two case studies and compare her approach and our approach to demonstrate the similarities and differences between the two.

\subsection{Renewable Electricity in the UK}

\subsubsection{Case Description}

In order to comply with international environmental agreements, such as the Kyoto protocol, governments must ensure that a sufficient amount of electricity is produced with CO2-friendly technologies (renewable or green technologies) such as wind turbines, photovoltaic panels and hydro generators. Currently, due to high initial investments, producing electricity with green technologies is more expensive than producing electricity in the conventional way using fuel-based technologies.

In the UK, the first Renewable Obligation (RO) regulation came into force in April 2002. The law obliges all electricity suppliers in the UK, to generate a certain proportion of their electricity from renewable sources. In $2006 / 07$ this proportion was $6.7 \%$ of the total production.

Suppliers are obliged to buy a certain proportion of the total electricity which they supply to their customers from renewable electricity producers. A government agency, the Office of Gas and Electricity Markets (OFGEM) is responsible for the enforcement of the $\mathrm{RO}$ regulation. If a supplier does not cover its obligation, it must make a deposit into a buy-out fund. The buy-out fee is a fixed price per $\mathrm{MW} / \mathrm{h}$ shortfall and is adjusted in line with the Retail Prices Index each year.

The value model of this case is shown in Figure 9-1. According to this model, a Customer pays some money to a Supplier for providing electricity. To be able to provide electricity to its customers, Supplier buys electricity from non-renewable and renewable electricity producers. Because the model depicts an ideal situation, all actors are assumed to be trustworthy and act according to the rules and agreements. Therefore, Supplier is assumed to buy a certain proportion of the electricity that it provides to its customers from renewable electricity producers and provide OFGEM with enough documents that prove its compliance with RO regulation. Upon buying Green Electricity, the supplier reports the supply of green electricity to OFGEM and receives an acknowledgement of compliance with the $\mathrm{RO}$ regulation.

Because green electricity is more expensive than regular elec- 


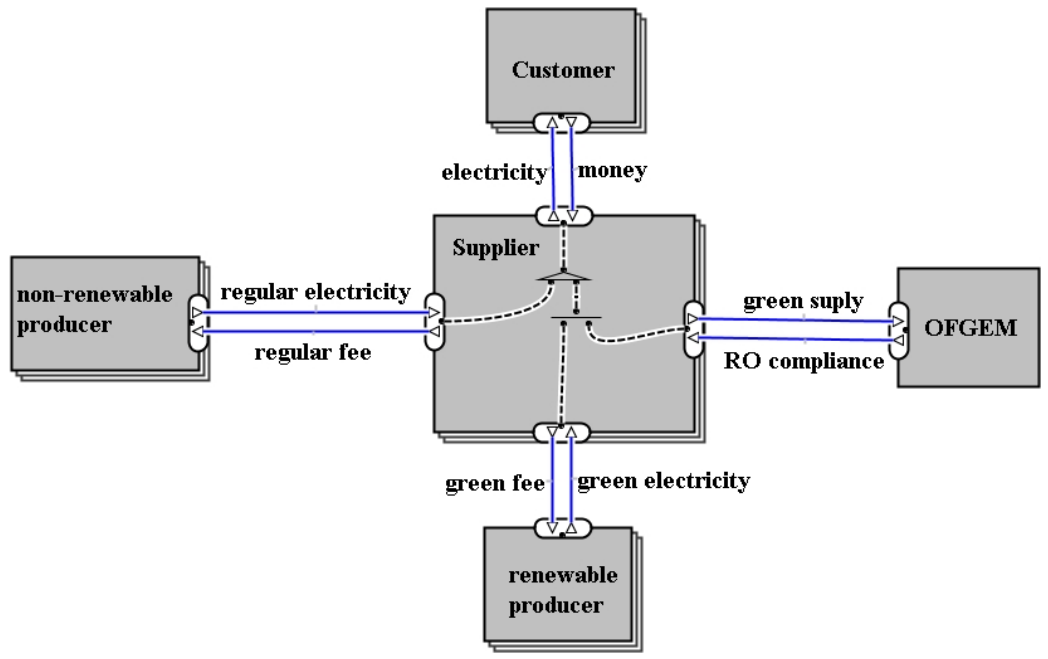

Figure 9-1 Electricity provision

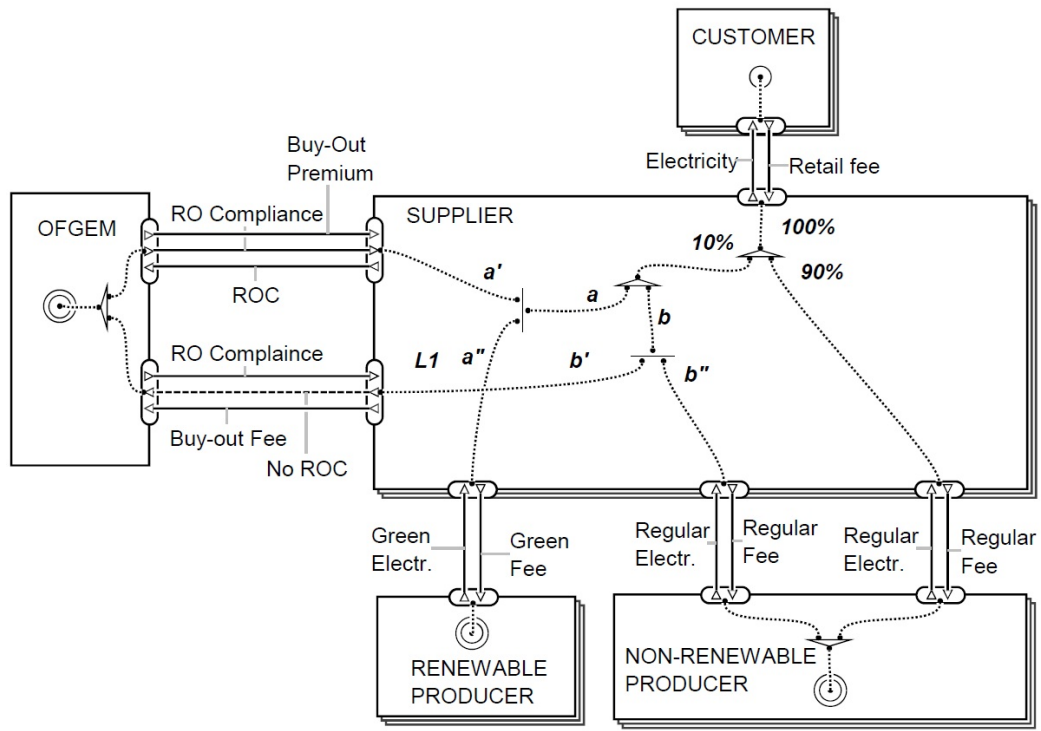

Figure 9-2 Kartseva's solution for electricity provision (adapted from [40]) 
tricity, suppliers have a tendency to buy less green electricity than the specified percentage. The goal is to reduce the probability of suppliers not acting trustfully which can be either by overstating the amount of the provided green electricity or understating the amount of the total provided electricity. Here we analyze the case using both Kartseva's method and our approach.

\subsection{2 $E^{3}$ control Approach for Electricity Provision}

After analyzing the case with her approach, Kartseva designed some $e^{3}$ control models that include all the scenarios in which an actor does not act trustfully or reciprocally. Figure 9-2 shows one of those models. The simple value model shown in Figure 9-1 has turned into a model which is quite complicated. She adds a new value transfer, indicating a penalty, as an outgoing value object of the counter actor Supplier in the sub-ideal path L1. She adds it to the transfer of No RO Compliance and Regular Supply, and change No RO Compliance to $R O$ Compliance.

$R O C$ and No $R O C$ represent the value objects Green Supply and No Green Supply, respectively. The penalty is represented by a value transfer Buy-out Fee. So, at the sub-ideal path b, where the supplier does not supply enough green electricity, he is obliged to pay a buy-out fee in order to cover the $R O$. The buy-out premiums paid by OFGEM as an incentive to compliant suppliers. As a result, the incentive value object Buy-Out Premium is added to the transfer of Green Supply and RO Compliance in Figure 9-1. In Figure 9-1, 10\% indicates the green electricity percentage. For more details regarding the $e^{3}$ control methodology and the way in which the model shown in Figure 9-2 is interpreted, interested readers are referred to Kartseva's thesis [40]

\subsubsection{Our approach for electricity provision}

In our approach, first of all we should identify those collaboration exchanges which are subject to trust. There are the cases where an actor is doing business with another actor, and the first actor trusts the second one and assumes that he will act reciprocally according to the value model and respects all the agreements. In fact this can be the case in any business collaboration, however here we only concentrate on some specific exchanges.

In normal cases, an actor needs to execute all its value exchanges in order to be able to provide its service or product to its customers. For example, a web shop which sells computers needs to buy a computer from another actor before being able to sell it 
to a customer. However, the most interesting cases are those in which two actors collaborate with each other in a value exchange; one actor (the trustor) trusts the other one (the trustee) and the trustee does not need to receive anything from the trustor, i.e. it can achieve its business goals without receiving any value object from the trustor. This type of value exchanges are mostly of compliance nature. A business actor should acquire a permission or consent from another actor to produce a value object but it does not.

In order to control the trustee in those special cases, based on the case, there might be numerous techniques. One such technique is to check once in a while to check the compliance of the trustee. We assume that the trustor can design a specific way for executing an inspection and he also can estimate the cost of the inspection. We also assume that the penalties, which the trustee should pay in case his non-compliance is proved, are already determined.

The payoff table of the compliance game between OFGEM and supplier is shown in Figure 9-3. Rows represent the strategies of OFGEM and columns those of Supplier. Each strategy combination defines a payoff pair, like $(65000,-80000)$ for (Inspect,Cheat), which is given in the respective table entry. Each cell of the table shows the payoff to OFGEM at the (lower) left, and the payoff to Supplier at the (right) top. The unit of calculation is assumed to be one month and the payoffs are in euro (€).

According to the payoff table, the base situation in which OFGEM does not do any inspection in one month and Supplier acts according to $\mathrm{RO}$ regulation in that month, gives the resulting payoff 0 to both OFGEM and Supplier. This situation corresponds to the assumptions of value model and it is interpreted as follows: if both actors (OFGEM and Supplier) act according to the agreements none of them earns anything extra and also none of them loses anything compared to what is agreed upon in the value model.

If OFGEM does not inspect and Supplier cheats, they receive payoffs $€-10000$ and $€ 20000$, respectively. This situation in this particular case is quite exceptional, and the payoffs are subject to a lot of discussion. Firstly, the payoff of Supplier is dependent on the percentage of the provided green electricity. The less green electricity is provided the more profit Supplier earns hence, $€ 20000$ represents an average profit. Secondly, payoff $€-10000$ might not be reasonable for OFGEM. If a supplier does not respect the agreements, it makes the air more polluted but it might not necessarily imply a loss for OFGEM. In fact, in this case, it 


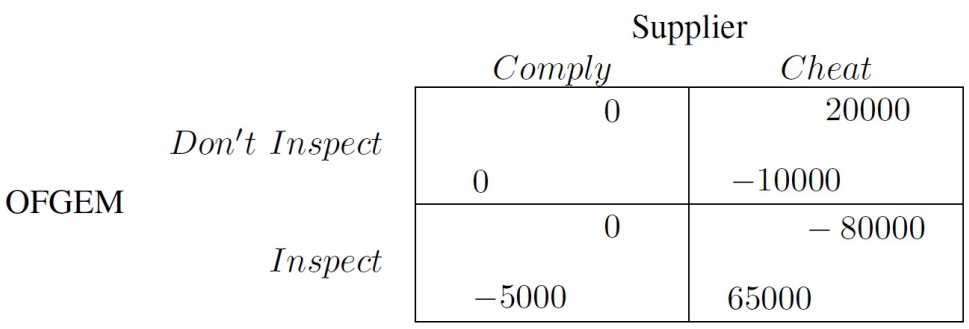

Figure 9-3 Compliance inspection game between OFGEM and Supplier.

would be fair to assume that OFGM represents society. It would have some social and societal consequences, and assigning an exact monetary value to those consequences is a real challenge.

If OFGEM does inspect and Supplier complies with the RO regulation, then the payoff of Supplier would still be 0 while the payoff of OFGEM is $€-5000$. This is because we assumed that the cost of inspection is $€ 5000$. In case Supplier does not act fully according to the RO regulation and OFGEM inspects, then OFGEM can put a high penalty $(€-80000)$ on Supplier. The amount of penalty is dependent on the percentage of provided green electricity therefore, $€-80000$ represents an average penalty. The payoff of OFGEM in this case is $€ 65000$ (€80000-€10000$€ 5000$ ), which is based on the assumption mentioned above that non-compliance behavior of Supplier would cost OFGEM $€ 10000$.

\section{Solving the Game}

Now, the question here is 'which strategy should each player play?' We observed that none of the players can play a strategy for ever because, for example, if OFGEM always chooses Don't Inspect, the best response for Supplier would be choosing Cheat strategy and this yields payoffs $€-10000$ and $€ 20000$ for OFGEM and Supplier, respectively, which is to the detriment of OFGEM. If OFGEM always chooses Inspect, the best response for Supplier would be choosing Comply strategy with resulting payoffs $€-5000$ and $€ 0$ for OFGEM and Supplier respectively, again to the detriment of OFGEM. On the other hand, if Supplier settles on Comply, OFGEM would choose Don't Inspect with resulting payoffs $€ 0$ for both players, which is to the detriment of Supplier since if OFGEM does not inspect, Supplier would be better off by simply cheating and getting payoff $€ 20000$ instead of $€ 0$. If Supplier settles on the Cheat strategy, OFGEM would prefer Inspect with resulting payoffs $€-80000$ and $€ 65000$ for Supplier and OFGEM, 
respectively, which is again to the detriment of Receiver. Therefore, if player $\mathrm{X}$ settles on a deterministic choice, player $\mathrm{Y}$ can play in its own interest and to the detriment of player $\mathrm{X}$.

According to the max-min strategy, in which each player tries to choose the strategy with the best worst payoff, i.e. the strategy by which the other party can impose less harm, players try to avoid those strategies which might cause big harms. The max-min strategy for OFGEM is to Inspect, since if OFGEM chooses the Inspect strategy then the worst case that might happen is the one in which Supplier chooses Comply with resulting payoff $€-5000$ for OFGEM, while if OFGEM chooses Don't Inspect strategy the worst case that might happen is the one in which Supplier chooses Cheat and that results in payoff $€-10000$ for OFGEM ( $€-5000$ is better than $€-10000)$.

Analogously, the max-min strategy for Supplier it is to choose Comply, since if Supplier chooses the Comply strategy then the worst case that might happen is the one in which OFGEM chooses Comply, which results in payoff $€ 0$ for Supplier, while if Supplier chooses Cheat the worst case is the one in which OFGEM chooses Inspect and that would result in payoff $€-80000$ for Supplier (€0 is better than $€-80000)$. Hence, if the actors apply the max-min strategy, OFGEM would choose the Inspect strategy, while Supplier would choose Comply. However, this is not a Nash equilibrium and hence not a stable recommendation to the two players, since if Supplier is complying with the regulation OFGEM would be better off by not inspecting. A pair of strategies, one for each player, forms a Nash equilibrium if even given knowledge of each other's strategies, neither player would have an incentive to switch to an alternate strategy [32]. Therefore, in this case if we treat each player as simply having the two strategies, then there is no Nash equilibrium for this game.

A mixed strategy for OFGEM is to inspect only with a certain probability. To find out which probability is the best lets examine two cases. Assume that the probability of inspection is $10 \%$, then, irrespective of that probability, Supplier gets payoff $€ 0$ when it complies, and expects payoff

$$
(0.9 *(20000))+(0.1 *(-80000))=10000
$$

when he cheats. Since the payoff in case of cheating $(€ 10000)$ is greater than payoff in case of complying $(€ 0)$, Supplier will still cheat, just as in the absence of inspection. If OFGEM increases the probability of inspection to, for example $30 \%$, then 
the expected payoff for Cheat is

$$
(0.7 *(20000))+(0.3 *(-80000))=-10000
$$

so, Supplier prefers to comply $(€ 0>€-10000)$. Therefore, if the inspection probability is either too low or too high, Supplier has a unique best response.

To find out which probabilities $O F G E M$ should assign to its strategies (Inspect and Don't Inspect) so that Supplier would be indifferent between it strategies (Comply and Cheat), we should solve the following equation:

$$
(20000) *(x)+(-80000) *(1-x)=0
$$

where $\mathrm{x}$ represents the probability of Don't Inspect strategy. Solving the equation results in $\mathrm{x}=0.80$. Hence the probability of Don't Inspect strategy would be $80 \%$ and consequently the probability of Inspect strategy would be $20 \%$.

Analogously to find out which probabilities Supplier should assign to its strategies (Comply and Cheat) so that OFGEM would be indifferent between it strategies (Inspect and Don't Inspect), we should solve the following equation:

$$
(-10000) *(1-x)=(-5000) *(x)+(65000) *(1-x)
$$

where $\mathrm{x}$ represents the probability of Comply strategy. Solving the equation results in $\mathrm{x}=0.9375$. Hence the probability of Comply strategy would be $93.75 \%$ and consequently the probability of Cheat strategy would be $6.25 \%$.

If Supplier was indifferent (i.e., both strategies give him the same payoff), he could choose the strategies randomly. The Supplier is indifferent if and only if OFGEM chooses Inspect with probability 0.2 , and Don't Inspect with probability 0.8 because then the expected payoffs for cheat is

$$
(0.8 *(20000))+(0.2 *(-80000))=0
$$

which is the same for Comply. This mixed strategy of OFGEM, makes Supplier indifferent between his strategies.

If OFGEM was indifferent (i.e., both strategies give him the same payoff), he could choose his strategies randomly. OFGEM is indifferent if and only if Supplier chooses Comply with probability 0.9375 and Cheat with probability 0.0625 , because then the expected payoffs for Don't Inspect

$$
(0.9375 *(0))+(0.0625 *(-10000))=-625
$$


and for Inspect

$$
(0.9375 *(-5000))+(0.0625 *(65000))=-625
$$

are the same.

According to the above discussion the mix strategies (choosing Inspect strategy $20 \%$ of the times and Don't Inspect strategy $80 \%$ of the times by OFGEM and choosing Cheat strategy $6.25 \%$ of the times and Comply strategy $93.75 \%$ of the times by Supplier) make the only Nash equilibrium of the game. The resulting expected payoffs are $€-625$ for OFGEM and $€ 0$ for Supplier.

After designing the value model, each business actor analyzes the collaboration and tries to estimate the amount of profit which it can expect from the collaboration. Similarly, in this case, Supplier and OFGEM, like other actors, estimate their profit in the collaboration. Then, these two actors should include the results of the game into the calculations. As shown above the expected payoff of OFGEM in the game is $€-625$. This means that each month OFGEM earns $€ 625$ less than what is expected in the ideal situation.

\subsection{Excise collection within the EU}

\subsubsection{Case Description}

The compliance game is a recurrent pattern in business collaboration. Here we explain another case study briefly which is again taken from Kartseva's thesis [40] and it also manifests an inspection game. The goal is twofold: 1) to demonstrate that the inspection game is a recurrent pattern in real life business, and 2) to show the generalizability and applicability of our approach for analyzing this type of situation.

An excise is a tax levied on the manufacture or sale of a product within a country. For some special goods, such as beer and cigarettes, the seller must pay this special excise tax. Within the EU, sellers must pay excise only in the country in which the excise good is consumed. Currently, different countries levi different excise tariffs on the same product. Therefore, if a beer producer in the Netherlands exports beer to a retailer in the UK, and the beer is consumed within UK, then the beer producer must pay the excise duty in the UK. Since there are officially no borders between the EU member states, the Dutch beer producer or seller is not obliged to report exported goods to Dutch Tax and Customs Administration (Customs NL). Figure 9-4 shows the value model 
of selling beer in an ideal situation.

Currently, the core evidence of export in the EU is the paper Administrative Accompanying Document (AAD). The AAD contains information about goods in the associated container or truck. According to the law, a Dutch beer company (e.g. BeerCo NL) should receive excise exemption upon presenting the AAD stamped by Customs UK [40]. However, beer producers or sellers could benefit from selling their beer in the Netherlands, and pretend that they have exported to a country that levies less tax on beer and, consequently, pay less tax. Table $9-1$ shows the excise rates in the Netherlands, Belgium and Germany. The big difference between the excise rate, specially between the Netherlands and Germany, tempts the beer producers to sell their products in the Netherlands and pretend that they have exported it to Germany.

One solution for Customs NL is to trust the reports of the producers or sellers, and run inspections once in a while to check the compliance of the producers or sellers. In the Netherlands, Customs NL periodically checks the excise declarations of the producers or sellers, and in practice, verification is often not done at all because it is labour intensive [40].

Table 9-1

Excise rate in some European countries

\begin{tabular}{|c|c|}
\hline Country & Excise per crate of beer \\
\hline the Netherlands & $€ 2.35$ \\
\hline Belgium & $€ 1.48$ \\
\hline Germany & $€ 0.68$ \\
\hline
\end{tabular}

\subsection{2 $e^{3}$ control Approach}

Figures 9-5 and 9-6 show two different control value solutions adapted from [40]. In fact, strictly speaking, these models are not the same value model as the ideal value model shown in Figure 9-4, because they contain some extra business actors such as TTP in Figure 9-5 and Customs UK in Figure 9-6. Note also that those actors who are from the UK are not represented in detail in the ideal value model shown in Figure 9-4.

We do not explain the details of these two solutions here. In short, each solution depicts a new value model in which a control mechanism is enforced using some evidence documents and some third-party business actors. Again here, like in Section 9.2, Kartseva does not provide the financial analysis, the result and impact of the solutions. 


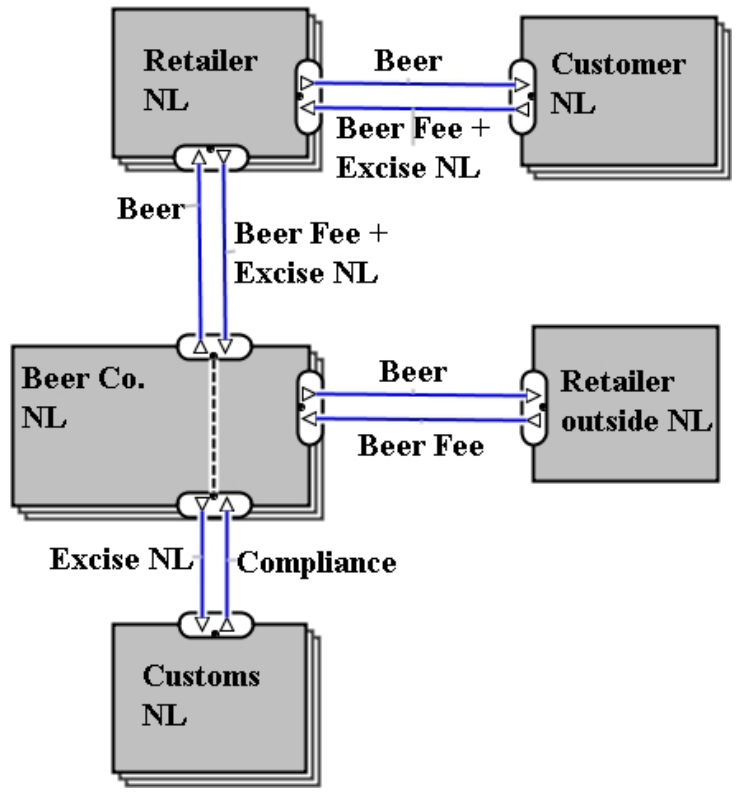

Figure 9-4 Ideal value model of the beer sales

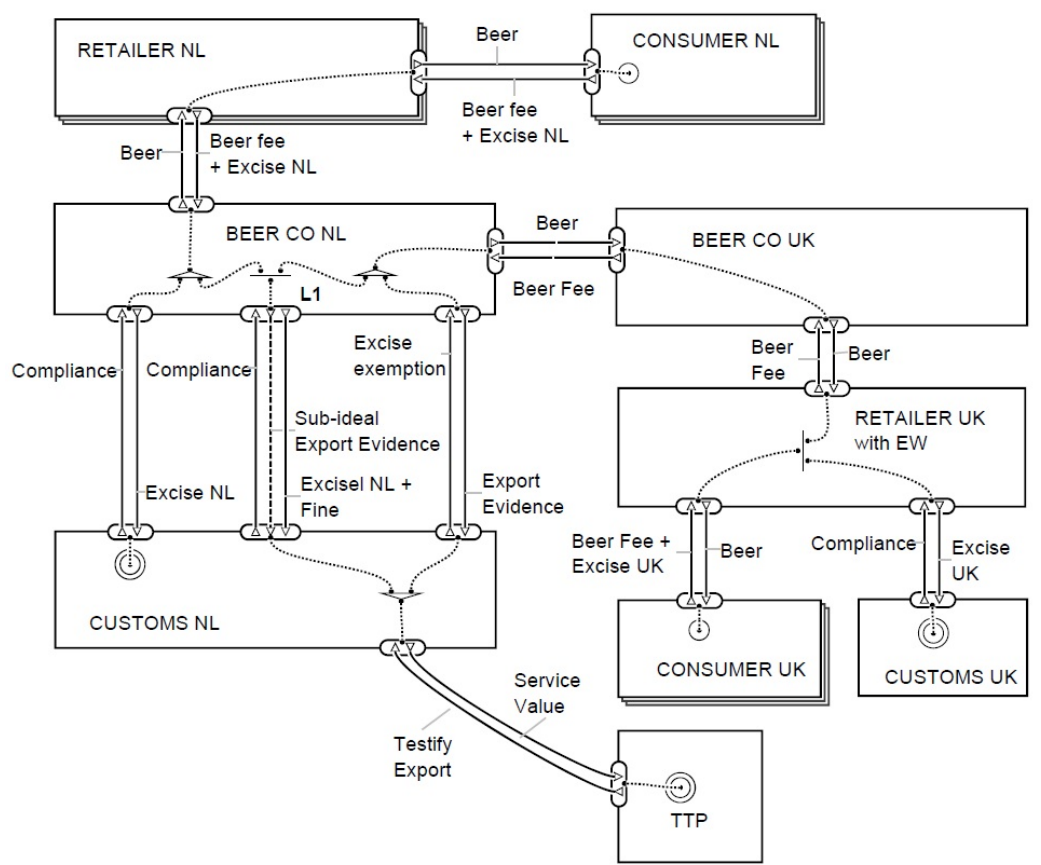

Figure 9-5 A control value model for the beer sales (adapted from [40]) 


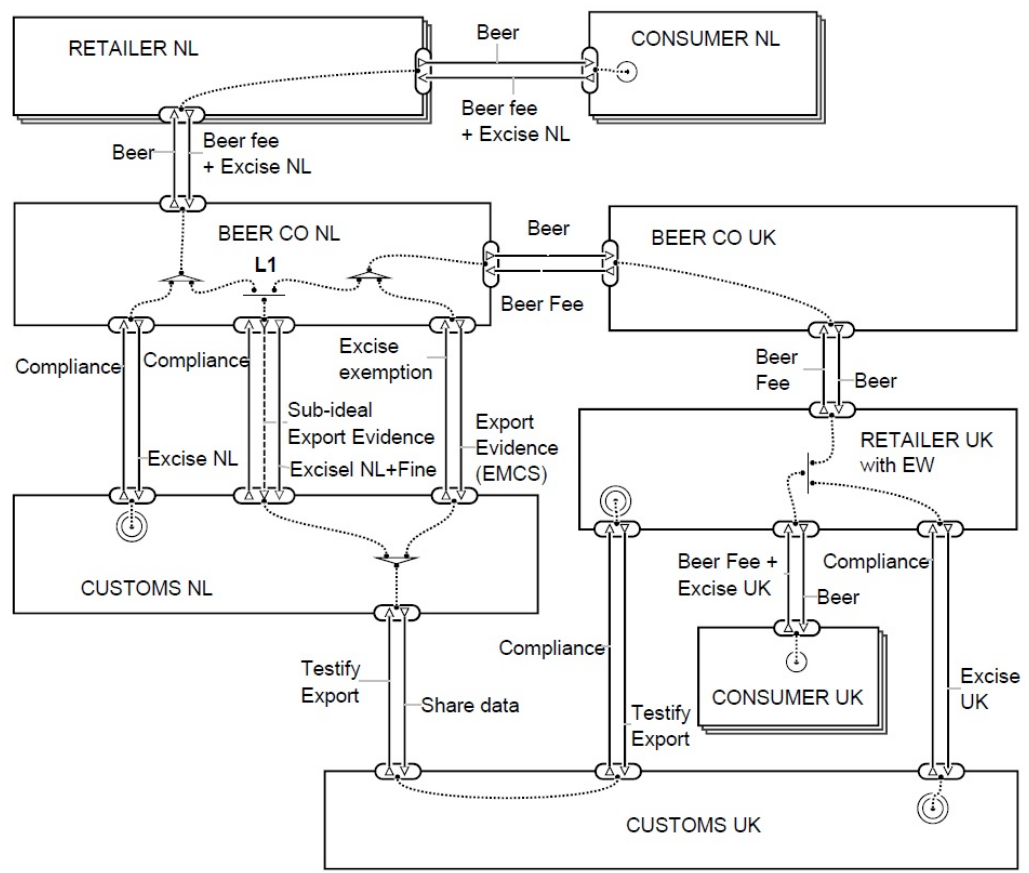

Figure 9-6 A control value model for the beer sales (adapted from [40])

Beer Co. NL

\begin{tabular}{|c|c|c|c|}
\hline \multirow{5}{*}{ Customs NL } & \multirow{3}{*}{ Don't Inspect } & Comply & Cheat \\
\hline & & 0 & 2000 \\
\hline & & 0 & -2000 \\
\hline & \multirow{2}{*}{ Inspect } & 0 & -20000 \\
\hline & & & 19000 \\
\hline
\end{tabular}

Figure 9-7 Compliance inspection game between Customs NL and Beer Co. NL. 


\subsubsection{Our Approach}

From the point of view of our approach the situation in this case is similar to the case discussed in Section 9.2. Again here we assume a payoff table for the inspection game, which is shown in Figure 9-7. The unit of measurement in the payoff table is assumed to be a truck, and therefore it indicates that the excise levied on a truck of bier is $€ 2000$. It also assumes that if Customs $N L$ want to employ an inspection method that inspects all the trucks that export beer, it would averagely cost $€ 1000$ per truck.

Due to the high similarity to the case discussed in Section 9.2, we do not explain the analysis of the game in detail, instead we only provide the result of the game.

To find out which probabilities Customs $N L$ should assign to its strategies (Inspect and Don't Inspect) so that Beer Co. NL would be indifferent between it strategies (Comply and Cheat), we should solve the following equation:

$$
(2000) *(x)+(-20000) *(1-x)=0
$$

where x represents the probability of Don't Inspect strategy. Solving the equation results in $\mathrm{x}=0.9090$. Hence the probability of Don't Inspect strategy would be $90.90 \%$ and consequently the probability of Inspect strategy would be $9.09 \%$.

Analogously, to find out which probabilities Beer Co. NL should assign to its strategies (Comply and Cheat) so that Customs $N L$ would be indifferent between it strategies (Inspect and Don't Inspect), we should solve the following equation:

$$
(-2000) *(1-x)=(-1000) *(x)+(19000) *(1-x)
$$

where $\mathrm{x}$ represents the probability of Comply strategy. Solving the equation results in $\mathrm{x}=0.9545$. Hence the probability of Comply strategy would be $95.45 \%$ and consequently the probability of Cheat strategy would be $4.54 \%$.

Using the above probabilities the final resulting payoffs of the Beer Co. NL and Customs NL are $€ 0$ and $€-90.90$, which means that Beer Co. $N L$ will earn as much as agreed upon in the value model, while Customs $N L$ earns averagely $€ 90.90$ less than what is expected. By comparing the result of the inspection game with other possible solutions, actors can choose the solution which gives them the best result. 


\subsubsection{Comparing the Two Approaches}

In contrast with Kartseva's approach in which all violations and punishments are explicitly modeled as exchanges, in our approach the focus is on the outcome and the financial result of the nonreciprocity behaviour of the trustee. By introducing the concept of sub-ideal value object, Kartseva tries to identify all those combinations in which a value object with less value than what is agreed upon (sub-ideal value object) is exchanged.

In some cases, there might be more than one actor who can act non-reciprocally, and in that case analyzing the situation with Kartseva's approach produces quite complicated models. This is not inline with the basic principle of the $e^{3}$ value methodology, which is supposed to be a lightweight approach with models that are easy to understand even for non experts.

Like in the previous section, in this section, again in contrast to Kartseva's approach, we analyzed each value exchange which is subject to non-reciprocally behaviour separately and we did not change the value model. Those actors who are not subject to nonreciprocity behaviour are supposed to be either trustworthy (i.e., act reciprocally) or the value exchanges in which they are involved in, are secure enough so that the trustee can not commit fraud. Our approach provides the actors with a more precise estimation of the profitability of the collaboration. Using our approach, the trustor can analyze any inspection option and estimate the financial impact and outcome of each option and then based on the result of the analysis, the trustor decides on the option which gives him the best payoff.

By comparing the result of the inspection game with other possible solutions, actors can choose the solution which gives them the best result. If none of the solutions gives a financially acceptable result it means that the value model is not realizable and consequently it should be replaced with another value model. In case of the inspection game, by changing the payoff numbers the results can be adapted therefore, business actors might be able to agree on a payoff table that makes the result acceptable for both actors.

In this case also despite their differences, the two approaches can be seen as complementary approaches because Kartseva's approach tries to identify all control mechanisms including those who involve some kind of inspection and punishment in case of fraud, while our approach analyzes the financial impact and the outcome of the collaboration using the inspection-punishment control mechanism. 


\subsection{Creating Value for All: Community-Based Tourism}

The case studies we discuss in Sections 9.2 and 9.3 are of particular nature in which an actor have to trust another actor and to prevent misusing that trust, the trusting actor needs to run inspections. Those cases manifest a game situation in a business collaboration and therefore, based on our findings in Chapter 7, we analyzed them using the concepts and techniques of game theory. Now we discuss another business collaboration case that also involves trust, however, strictly speaking there is no game in those collaborations. This case study is to demonstrate the applicability of our findings of Chapter 6.

\subsubsection{Case Description}

In Kyrgyzstan, Swiss Association for International Cooperation, Helvetas, initiated Community-Based Tourism (CBT) in rural areas. The simple idea, which was well supported by local communities, was that with the help of $C B T$ groups, individual service providers provide specific services to the Tourists. The first $C B T$ group was established in May 2000 in Kochkor village, and until 2010 the number of CBT organizations grew to eighteen ${ }^{1}$.

$C B T$ groups consist of local tourism service providers (individuals and families from the local community) and representatives of Local Authorities. CBT service providers include drivers, guides and owners of guesthouse, yurts and horses. Local tourism service providers represent small tourism enterprises specialized in providing different types of tourism services, such as accommodation in houses or yurts, guides, transport services or horse rentals, etc. The CBT groups also control the quality of tourism services using quality control committees, promote certain tourist destinations, and raise awareness among the local population on environmental issues by organizing events and round tables, and giving lectures at schools.

CBT groups provide some services to Tourists and Tour Operators, such as booking services for home-stays, yurtas/jailoo tourism and tracking, guided tours of cultural and historic sights, handicraft demonstrations and sales, car rentals and miscellaneous tourist information. The main objective of $C B T$ groups in Kyrgyzstan is to promote sustainable Community-based ecotourism services that offer Tourists unique experiences, generate income for rural families, and preserve the natural and cultural heritage

\footnotetext{
${ }^{1}$ http://cases.growinginclusivemarkets.org/documents/33
} 


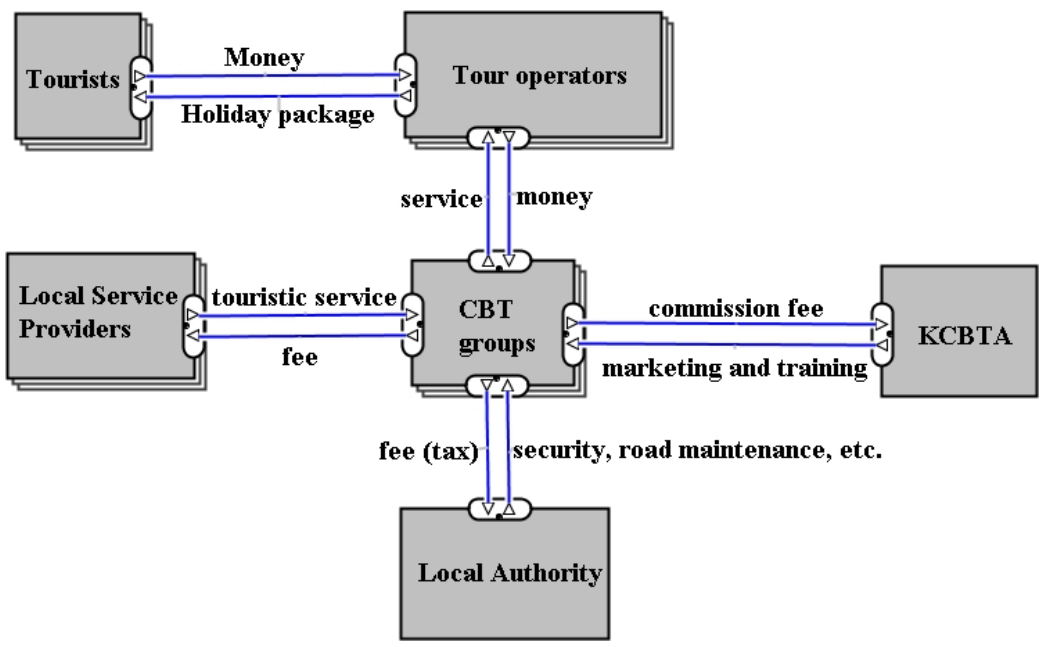

Figure 9-8 Community-Based Tourism (CBT) in Kyrgyzstan

of the country.

Besides $C B T$ groups, there are two main actors involved in the CBT business model: Tour Operators (TO) and Kyrgyz CommunityBased Tourism Association (KCBTA). Tour Operators sell tour packages and products (national games, carpet making shows, etc.) to foreign Tourists, and direct them to CBT groups. KCBTA is an umbrella organization formed by local CBT groups in 2003 for further development and coordination of tourist activities within the country. The association provides marketing and training services to its members and represents their interests at the national level. $K C B T A$ provides accreditation of the tourism services once a year with the involvement of Tour Operators and CBT groups. The main income sources of KCBTA are the following: income from training, sales of tourist souvenirs and products, membership fees, commissions from booking activities and rental of tourism equipment.

Some Local Authorities contribute also to the project by providing some services, such as maintenance of roads and public facilities, providing security, etc. The local authorities are governmental organizations and therefore they receive tax.

\subsubsection{Value Model}

The value model of this case is shown in Figure 9-8. This model shows a business collaboration between a group of business actors. Trust plays a major role in this collaboration but, strictly speaking 
there is no game relation between any two actors. However, since $C B T$ groups control the quality of the services provided by the Local Service Providers, we can analyze the collaboration between these two actors as a game.

If a $C B T$ group inspects to check the quality of a service provided by a Local Service Provider and it turns out that the provided service has sufficient quality, then the inspection would be a waste of money for CBT group. However, if the quality of the service provided by the Local Service Provider does not have the satisfying quality, the trust of CBT group in the Local Service Provider decreases. If the trust of a $C B T$ group in a Local Service Provider drops below some pre-defined level that Local Service Provider is put out of business.

\subsubsection{Trust Model}

The collaboration between a Local Service Provider and the $C B T$ group does not manifest a pure game, because if it were a pure game, $C B T$ groups would not have any control on the quality of the services provided by the Local Service Providers, i.e. CBT groups would not have known how Local Service Providers are doing unless they had inspection. However, in reality $C B T$ groups can receive feedback from Tourists and they can decide on the collaboration with a Local Service Provider based on this feedback.

We assume that the trust relations between the business actors of CBT case are as shown in Figure 9-9. According to this model, there is a mutual trust relation between Tour Operators and $C B T$ groups, Local Authorities and CBT groups and also KCBTA and $C B T$ groups. However, there is a one-way trust relation from Tourists to Tour Operators and from Local Service Providers to CBT groups.

If there is no trust relation in the trust model between two actors which are in direct relation with each other in the value model (e.g. Tour Operators to Tourists), then there is not enough trust in that particular direction between actors (Tour Operators do not have sufficient trust in Tourists). We recall that this only means Tour Operators should receive Money from Tourists before providing any service to them.

Not having a trust relation in the trust model between two actors which are not in direct relation with each other in the value model (e.g. Tour Operators and $K C B T A$ ), does not mean there is no trust relation between them. It is only because the trust model only shows the trust relations between those actors who are in direct relation with each other in the value model. 


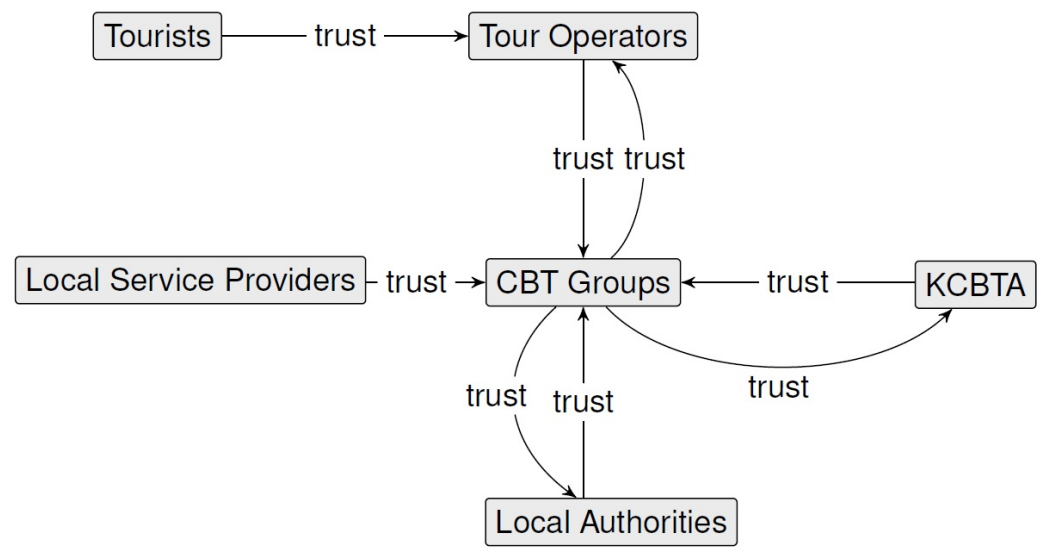

Figure 9-9 The trust model of CBT case

Table 9-2 Initial trust table

\begin{tabular}{|c|c|c|c|c|c|c|}
\hline & Tourists & TO & LSP & CBT & LA & KCBTA \\
\hline Tourists & & 1 & & & & \\
\hline Tour Operators (TO) & & & & 1 & & \\
\hline Local Service Providers (LSP) & & & & 1 & & \\
\hline CBT groups (CBT) & & 1 & & & 1 & 1 \\
\hline Local Authorities (LA) & & & & 1 & & \\
\hline KCBTA & & & & 1 & & \\
\hline
\end{tabular}

The initial values of trust relations and the weights of others's opinion regarding the trustworthiness of an actor for each actor are shown in Tables 9-2 and 9-3. In this case, we assume that at the beginning of a collaboration every actor assigns 1 to all its trust relations. The business actors start the collaboration using the current trust relations.

Now assume that at some point in time during the collaboration, a Tour Operator is not satisfied with the service it receives from a CBT group (in terms of trust this means Tour Operator expected to receive a value object worth $\mathrm{V}$ but it receives a value object with less value). Due to that, Tour Operator reduces the value of its trust in the CBT group from 1 to 0.8 . This new situation is shown in Table 9-4.

Table 9-3

Table of

Weights

\begin{tabular}{|c|c|c|c|c|c|c|}
\hline & Tourists & TO & LSP & CBT & LA & KCBTA \\
\hline Tourists & 0.8 & 0 & 0 & 0.2 & 0 & 0 \\
\hline Tour Operators (TO) & 0 & 0.5 & 0.3 & 0 & 0.1 & 0.1 \\
\hline Local Service Providers (LSP) & 0 & 0.2 & 0.6 & 0 & 0.1 & 0.1 \\
\hline CBT groups (CBT) & 0 & 0 & 0 & 1 & 0 & 0 \\
\hline Local Authorities (LA) & 0 & 0 & 0 & 0 & 0.8 & 0.2 \\
\hline KCBTA & 0 & 0.1 & 0.1 & 0 & 0.1 & 0.7 \\
\hline
\end{tabular}


Table 9-4

Trust table after the

first

incident

\begin{tabular}{|c|c|c|c|c|c|c|}
\hline & Tourists & TO & LSP & CBT & LA & KCBTA \\
\hline Tourists & & 1 & & & & \\
\hline Tour Operators (TO) & & & & 0.8 & & \\
\hline Local Service Providers (LSP) & & & & 1 & & \\
\hline CBT groups (CBT) & & 1 & & & 1 & 1 \\
\hline Local Authorities (LA) & & & & 1 & & \\
\hline KCBTA & & & & 1 & & \\
\hline
\end{tabular}

Table 9-5

Trust table after after the first transaction after the first incident

\begin{tabular}{|c|c|c|c|c|c|c|}
\hline & Tourists & TO & LSP & CBT & LA & KCBTA \\
\hline Tourists & & 1 & & & & \\
\hline Tour Operators (TO) & & & & 0.900 & & \\
\hline Local Service Providers (LSP) & & & & 0.960 & & \\
\hline CBT groups (CBT) & & 1 & & & 1 & 1 \\
\hline Local Authorities (LA) & & & & 1 & & \\
\hline KCBTA & & & & 0.980 & & \\
\hline
\end{tabular}

Table 9-6

Trust table after after the second transaction after the

first

incident

\begin{tabular}{|c|c|c|c|c|c|c|}
\hline & Tourists & TO & LSP & CBT & LA & KCBTA \\
\hline Tourists & & 1 & & & & \\
\hline Tour Operators (TO) & & & & 0.936 & & \\
\hline Local Service Providers (LSP) & & & & 0.954 & & \\
\hline CBT groups (CBT) & & 1 & & & 1 & 1 \\
\hline Local Authorities (LA) & & & & 0.996 & & \\
\hline KCBTA & & & & 0.972 & & \\
\hline
\end{tabular}

Table 9-7

Trust table after after 15

transaction after the

first incident

\begin{tabular}{|c|c|c|c|c|c|c|}
\hline & Tourists & TO & LSP & CBT & LA & KCBTA \\
\hline Tourists & & 1 & & & & \\
\hline Tour Operators (TO) & & & & 0.971 & & \\
\hline Local Service Providers (LSP) & & & & 0.971 & & \\
\hline CBT groups (CBT) & & 1 & & & 1 & 1 \\
\hline Local Authorities (LA) & & & & 0.971 & & \\
\hline KCBTA & & & & 0.971 & & \\
\hline
\end{tabular}


Table 9-8 Trust table after the second incident

Table 9-9 Trust table first transaction after the second incident after the

\begin{tabular}{|c|c|c|c|c|c|c|}
\hline & Tourists & TO & LSP & CBT & LA & KCBTA \\
\hline Tourists & & 1 & & & & \\
\hline Tour Operators (TO) & & & & 0.971 & & \\
\hline Local Service Providers (LSP) & & & & 0.75 & & \\
\hline CBT groups (CBT) & & 1 & & & 1 & 1 \\
\hline Local Authorities (LA) & & & & 0.971 & & \\
\hline KCBTA & & & & 0.971 & & \\
\hline
\end{tabular}

\begin{tabular}{|c|c|c|c|c|c|c|}
\hline & Tourists & TO & LSP & CBT & LA & KCBTA \\
\hline Tourists & & 1 & & & & \\
\hline Tour Operators (TO) & & & & 0.904 & & \\
\hline Local Service Providers (LSP) & & & & 0.838 & & \\
\hline CBT groups (CBT) & & 1 & & & 1 & 1 \\
\hline Local Authorities (LA) & & & & 0.971 & & \\
\hline KCBTA & & & & 0.948 & & \\
\hline
\end{tabular}

After a Tour Operator reduces its trust in a $C B T$ group from 1 to 0.8 , the collaboration ends if 0.8 is bellow the acceptable level for this Tour Operator. However, here we assume that the trust level is still satisfactory and consequently the collaboration continues. In each transaction, before starting a value exchange, each actor calculate the new trust value according to Formula 6.3, and executes the value exchange if and only if the computed trust level is still above the threshold. The computed trust value of the trust relation is set as the new value of that trust relation. In fact, actors can assign any value to a trust relation, however, to show the mechanism of the computations we assume that all actors use the calculated value of trust relations according to Formula 6.3.

After the end of the first transaction, each actor updates its values in the trust table, and for this particular case the table of trust values after the first transaction is shown in Table 9-5. Table 9-6 depicts the trust table after the second transaction. This process continues, and the values of trust relations of all actors in $C B T$ group converge. In the case at hand, after 15 transactions we reach a fixed point in which the values of trust relations of all actors in CBT group are the same up to 3 decimal places.

Table 9-10 Trust table after the second transaction after the second incident

\begin{tabular}{|c|c|c|c|c|c|c|}
\hline & Tourists & TO & LSP & CBT & LA & KCBTA \\
\hline Tourists & & 1 & & & & \\
\hline Tour Operators (TO) & & & & 0.895 & & \\
\hline Local Service Providers (LSP) & & & & 0.876 & & \\
\hline CBT groups (CBT) & & 1 & & & 1 & 1 \\
\hline Local Authorities (LA) & & & & 0.966 & & \\
\hline KCBTA & & & & 0.935 & & \\
\hline
\end{tabular}


Table 9-11 Trust table after 24 transactions after the second incident

\begin{tabular}{|c|c|c|c|c|c|c|}
\hline & Tourists & TO & LSP & CBT & LA & KCBTA \\
\hline Tourists & & 1 & & & & \\
\hline Tour Operators (TO) & & & & 0.928 & & \\
\hline Local Service Providers (LSP) & & & & 0.928 & & \\
\hline CBT groups (CBT) & & 1 & & & 1 & 1 \\
\hline Local Authorities (LA) & & & & 0.928 & & \\
\hline KCBTA & & & & 0.928 & & \\
\hline
\end{tabular}

Now assume that later at some point in time during the collaboration, due to the unsatisfactory performance of $C B T$ group, Local Service Provider decreases the value of its trust in $C B T$ group to 0.75. This new situation is shown in Table 9-8. Again assume that 0.75 is above the threshold for the Local Service Provider. We assume that each actor sets the threshold values for its trust relations itself.

Again, after each transaction the new values of trust relations, calculated according to Formula 6.3 are assigned. The values of trust relations after the first, the second and the 24th transaction are shown in Tables 9-9, 9-10 and 9-11 respectively. As it can be seen in Table 9-11, after 24 transactions the values of trust relations of all actors in $C B T$ group are the same up to 3 decimal places.

The goal of this case study was to show the applicability and usability of the trust measurement method introduced in Chapter 6. Bellow we elaborate on some points manifested by this case study.

\subsubsection{Discussion}

The case described above exposes some interesting points which we will elaborate on more:

- Here we only discuss the trust computation using Formula 6.3 for $C B T$ group, because according to the Formula actor A computes the value of its trust in actor B based on its opinion, and the opinion of those actors who are in direct relation with actor B and trust B. Therefore, the trust calculation is useful only for those actors who are trusted by more than one actor in the collaboration. For example, $K C B T A$ is trusted only by $C B T$ group therefore, for computing its trust in $K C B T A$ using Formula 6.3, CBT group, has only its own opinion and, consequently, there is no point in using the formula. We can use Formula 6.3 to calculate the value of trust relation between Tourists and Tour Operators, however, for the sake of brevity we omitted this calculation. 
The more actors trust one particular actor in the collaboration, the more important that actor would be for the collaboration. Replacing an actor who is trusted by one actor in the collaboration is much easier than replacing an actor who is trusted by multiple actors in the collaboration. Hence, actors need to evaluate their trust in such actors with more scrutiny.

- When an actor loses the trust of one of its partner in the collaboration, that incident affects the trust of other actors in that actor. We saw that when Tour Operator decreased the value of its trust in $C B T$ group from 1 to 0.8 , it finally reduced the trust of all other actors (Local Service Provider, Local Authority and $K C B T A$ ) from 1 to 0.971 . This means that any wrong-doing action will remain as a bad reputation for the wrong-doer in the collaboration, unless the wrong doer does its best and respects all the agreements fully so that other actors assign a value higher than the calculated value to their trust in the wrong doer. Hence, after losing trust, regaining it is quite difficult. That is in line with the well-known expression which says "Trust is hard to get and easy to lose".

We also observed that after the first incident, and after the trust values converge, another incident happened and that reduced again the trust of $C B T$ group partners in $C B T$ group even more. Therefore, all wrong-doing actions have a cumulative effect.

- All actors who are in relation with one actor in the collaboration and trust that actor all get the same opinion regarding the trustworthiness of that actor.

- The reason behind computing the value of the trust of an actor in another actor using the values of the trust relations of other actors in that actor is because the collaborative network is a representative of team work and joint collaboration, and it survives if and only if there is at least one trust relation with satisfactory strength between every pair of business actors who are in direct relation with each other. If the value of a trust relation between two actor drops below the threshold, then those two actors can no longer collaborate in the current setting, and consequently the whole collaboration collapses. Therefore, when an actor trusts another actor in the collaboration, it is important for the trusting actor to take into account the way that the trustee is collaborating with other actors in the collaboration. 


\section{Conclusions}

"Science is organized knowledge. Wisdom is organized life." - Immanuel Kant (1724 - 1804)

This chapter summarizes the contribution of the thesis and enumerates some points for future research.

\subsection{Contributions}

In Section 1.2, we enumerated three main risks and challenges in designing collaborative networks. Those risks are:

1. Non-executability/non-feasibility risk.

2. Risk of untrustworthy partners.

3. Risk of non-profitability.

Accordingly, we also enumerated three main research problems in Section 1.3, which are:

- P1:

1. P1.1: How to generate a coordination model based on a value model?

2. P1.2: How to generate a value model based on a coordination model?

3. P1.3: How to define and check the consistency between a value model and a coordination model?

- P2: How to model the trustworthiness of business actors involved in a business collaboration so that actors can develop a better understanding regarding the trustworthiness of their partners in the collaboration and create a more stable and durable collaboration?

- P3: 
1. P3.1: How to analyze the profitability of unobservable value exchanges (a value exchange that needs inspection)?

2. P3.2: How to assess the profitability and sustainability of the collaborative networks by taking trust into account?

In this chapter we reflect on the results. The thesis can be divided into three parts; with each part addressing one of these three research problems. Consequently the contribution of the work in this thesis can also be divided into three parts. Now, we explain which research problem has been addressed in which chapter(s) and what is the contribution of each chapter.

\subsubsection{Non-executability/Non-feasibility Risk}

The issue of modeling a business collaboration from the value and coordination perspectives, and studying the relation between these two models address the risk of executability/feasability of the business collaboration. We investigated the relation between these two types of models in such a way that the findings and guidelines are general and applicable in different contexts. The first research problem is related to the non-executability/nonfeasibility issue in the design of the e-business networks. This section explains how we addressed the first research problem in this thesis.

\section{P1.1: How to generate a coordination model based on a value model?}

We addressed this research problem in Chapter 3. We proposed a general stepwise method for generating a BPMN coordination model from an $e^{3}$ value value model. The method is a patternbased method. First, it tries to identify the value patterns in the value model and then it adds a coordination pattern to the coordination model which realizes the value pattern. The chapter is based on two published papers [52] and [51].

Unlike previous works, our proposed method avoids complicated concepts like property right, physical delivery, etc. nor does it depend on intermediate models, but it still guides the modeler to a coordination model that is consistent with the value model. The generated coordination model is consistent with the base value model according to our consistency definition (3.1.3). 


\section{P1.2: How to generate a value model based on a coordination model?}

The motivation behind researching this problem is twofold. Firstly, before having a business collaboration case up and running, business actors might want to evaluate its performance and, based on the evaluation results, propose modifications to the collaboration. By abstracting from the coordination details and focusing on the value aspect of the collaboration, the value model generated from the coordination model of such a business collaboration helps the business actors understand the real economic nature of the collaboration, and it can also be used as a communication medium between different non-technical profit and loss responsible actors in the collaboration. Secondly, by combining this transformation with the value model to coordination model transformation, one has the ability to track the changes in one of the models into the other model in a consistent way.

This research problem is addressed in Chapter 4. The chapter is based on a published paper [53].

\section{P1.3: How to define and check the consistency be- tween a value model and a coordination model?}

We introduced a consistency definition in Section 3.1.3 and we used it to check the consistency of value and coordination models in the proposed transformation methods.

Chapter 5 is also related to the executability/feasability issue in the design of e-business networks. In that chapter we discussed the automation of value to coordination model transformation. To do that, first, we presented the source model as a graph and generated the target model by applying some transformation rules to the source graph. The transformation of an $e^{3}$ value model into a BPMN model consists of two parts: an automated part that produces a set of message patterns, followed by a manual part where an expert orders the messages and adds some further detail.

The transformation can not be done fully automatically because in general there is some information in the coordination model which is not in the value model, and this should be added by an expert. This chapter is based on a published paper [55].

\subsubsection{Risk of Untrustworthy Partners}

The risk in any business collaboration is that a partner will not behave according to the value model but, instead, acts in favor of its own goals, to the detriment of other partners' goals. Each 
partner will assess this risk and does a trade-off between (1) the cost of inspection and preventive measures to avoid opportunistic behaviors and (2) the risk of simply trusting others and assuming that they will not act opportunistically.

P2: How to model the trustworthiness of business actors involved in a business collaboration?

We addressed this research problem in Chapter 6. We first specified how trust is viewed in this thesis, based on the contributions reported in literature, and then extended the $e^{3}$ value business ontology with trust-related concepts. Later on, we proposed a formula to measure the trustworthiness of an actor in a collaborative network, based on the opinion of the direct partners of that actor. We applied the method on a real business case and later in Chapter 9 we provided additional validation by simulation cases. The chapter is based on a published paper [60].

\subsubsection{Risk of Non-profitability}

The ultimate goal of each business actor to participate in any business collaboration is to gain some profit. Hence, estimating the profitability of a collaborative network is of particular importance for business actors. $E^{3}$ value models depict an ideal situation in which all actors are assumed to act trustfully and consequently the profitability analysis in $e^{3}$ value methodology is valid only if all actors act trustfully and fulfil the agreements. This is one interpretation. Another interpretation is that the value assigned to value objects by an actor already incorporates (costs induced from) risks. However, in the latter case you cannot see how evolving trust relationships and preventive measures affect this value. In order to make the profitability analysis more precise we need to drop the trustworthiness assumption.

\section{P3.1: How to analyze the profitability of unobserv- able value exchange?}

This research problem is addressed in Chapter 7. In that chapter, we identified two types of value exchanges, namely "Observable" and "Unobservable" value exchanges in business collaborations. We also explained that in "Unobservable" value exchanges, one of the business actors needs to employ inspection (an activity which should be done at the receiver of a value object to verify the value transfer), which costs money, to verify if its partner is acting trustworthy and abiding by the agreements. In this particular 
case, the inspection corresponds to a strategy in a game, and therefore we used the concepts and techniques of the game theory to analyze the interplay between the two actors, to provide each actor with the real payoff or profit which it can earn out of the collaboration. The chapter is based on a published paper [102].

P3.2: How to assess the profitability and sustainability of the collaborative networks by taking trust into account?

This research problem is addressed in Chapter 8. In that chapter, we first discussed the endurability of a collaborative network using the trust relations between the business actors, and then introduced a method to refine the profitability calculations using the trust relations between the actors and the value (strength) of the trust relations. Providing the partners with value models supplemented with extra information regarding the endurability and profitability of the collaboration, enables them to decide on those collaborations which are more durable and profitable. Since value models depict the required exchanges to achieve a business goal, when they are enriched with information about trust, endurability and profitability, they can provide more insights to specify service level agreements among the participants within the network. This chapter is based on a published paper [108].

In Chapter 9 , we provided additional validation by simulation cases for the findings in Chapters 7 and 8 .

\subsection{Future Research}

Since the contribution of the thesis is divided into three parts, future research can also be done in three different directions.

\subsubsection{Modeling Collaborative Networks}

In terms of modeling collaborative networks from the value and coordination perspectives and the relation between these two aspects, our pattern-based value to coordination model transformation can be enriched by identifying more value and coordination patterns. Depending on the context of the collaboration and also depending on the requirements of the business actors, for each case, a specific coordination pattern can be recommended to the designer which suits the situation best. For example, we proposed a pair of request and response coordination messages to realize the 
transformation of a value object (actor $\mathrm{A}$ requests value object $\mathrm{V}$ from actor $B$ and then actor $B$ sends actor $A$ value object $V$ ). This is the general template which is composed of the minimum set of coordination messages, however in reality more rich and detailed message patterns, such as including a confirmation message, might be needed. For instance, after receiving a request from actor B, actor A first sends actor B a confirmation message, which can be in the form of a contract. Then by receiving the response of the confirmation message, actor A can be sure that the request is a valid request and is issued from a valid source/actor. These extra coordination messages can be provided as an option to the designer.

Money as a value object takes the lion share in the value objects, and roughly half of the value objects in a value model are related to money (payments) one way or the other. In this thesis, we use a normal pair of request-send coordination messages for realizing the payments, however, in the real world payment patterns could be of different forms, and they are of vital importance to business actors.

Different payment patterns not only make different coordination orders, but they also can affect the trust relations and profitability analysis of the collaboration. There is a huge difference between paying the whole sum of money at once and paying the money in parts. Likewise, paying at the end of the month is different from paying in advance or right after receiving a value object. Each payment pattern has its own characteristics and requires a special level of trust. Therefore, enriching the model transformation with different payment patterns and providing the business actors with detailed information of the payment and of the implications of each payment pattern is considered as an important extension of the current model.

A tool can also be developed to guide the designer through the modeling process. The tool can check the consistency between the two models and it can help the designer trace relations between these models and design mutually consistent models.

\subsubsection{Measuring Trust in Collaborative Networks}

Regarding measuring the trustworthiness of business actors in a collaborative network, the proposed model considers a number to quantify the trustworthiness value of an actor. In this sense, the trust value is used as an indication of the trustworthiness of the business actor in different aspects such as quality, price, delivery time, etc. Therefore, an extension of the model could be breaking 
the trust value into components based on the critical attributes of the goods or services (e.g. delay, quality, etc.) and compute the trustworthiness of the actors regarding each of these attributes separately. This is interesting because, on one hand, these critical attributes vary according to the product in question and, on the other hand, an attribute can be of different importance to different actors.

\subsubsection{Profitability Analysis in Collaborative Networks}

Taking appropriate measures to inspect and prevent violations in the value exchange process can affect the level of trust which collaboration actors have on each other. For example, an actor that does not trust another actor may consider implementing appropriate safeguards in order to increase the trust (decrease the risk). Therefore, enriching the trust analysis with safeguards, and providing the business actors with a trade-off between risks and profits associated with each option would be an interesting extension of the profitability analysis presented in this thesis. 


\section{References}

[1] Richard Normann and Rafael Ramírez. From value chain to value constellation: designing interactive strategy. Harvard Business Review, 71(4):65-77, July 1993.

[2] Eric T.T. Wong. Trust management in virtual product development networks. Encyclopedia of information science and technology, 2nd ed. Hershey, PA: IGI Global:3831-3839, 2009.

[3] Luis M. Camarinha-Matos and Hamideh Afsarmanesh. The emerging discipline of collaborative networks. In Virtual Enterprises and Collaborative Networks, pages 3-16, 2004.

[4] Luis M. Camarinha-Matos and Hamideh Afsarmanesh. Collaborative Networks: Reference Modeling. Springer Publishing Company, Incorporated, 1 edition, 2008.

[5] Don Tapscott, David Ticoll, and Alex Lowy. Digital Capital: Harnessing the Power of Business Webs. Harvard Business School Press, Boston, 2000.

[6] B. R. Katzy and G. Schuh. The virtual enterprise. in Handbook of Life Cycle Engineering: Concepts, Methods and Tools, 1997.

[7] Nitin Nayak, Kumar Bhaskaran, and Raja Das. Virtual enterprises - building blocks for dynamic e-business. Information Technology for Virtual Enterprises, Workshop on, 0:8087, 2001.

[8] J. Browne and J. Zhang. Extended and virtual enterprises - similarities and differences. International Journal of Agile Management Systems, pages 30-36, 1999.

[9] N. Mostert. Towards an extended enterprise through e-Business integration. Master thesis, Nelson Mandela Metropolitan University, 2004. 
[10] E.R. Rigsbee. Developing strategic alliances. Crisp Professional Series. Crisp Publications, 2000.

[11] David C. Mowery, Joanne E. Oxley, and Brian S. Silverman. Strategic alliances and interfirm knowledge transfer. Strategic Management Journal, 17:77-91, August 1996.

[12] Richard Normann and Rafael Ramírez. From value chain to value constellation: designing interactive strategy. Harvard Business Review, 71(4):65-77, July 1993.

[13] Richard Normann and Rafael Ramirez. Designing Interactive Strategy - From Value Chain to Value Constellation. John Wiley \& Sons Inc., Chichester, UK, 1994.

[14] Jaap Gordijn and Hans Akkermans. Value based requirements engineering: Exploring innovative e-commerce ideas. Requirements Engineering Journal, 8(2):114-134, 2003.

[15] Michael Eugene Porter. What is strategy?, volume 74. Harvard Business Review, November-December 1996.

[16] Jaap Gordijn, Hans Akkermans, and Hans van Vliet. Business modelling is not process modelling. In ER Workshops, pages $40-51,2000$.

[17] http://www.bpmn.org/.

[18] Vincent Pijpers and Jaap Gordijn. Bridging business value models and process models in aviation value webs via possession rights. In HICSS '07: Proceedings of the 40th Annual Hawaii International Conference on System Sciences, page 175a, Washington, DC, USA, 2007. IEEE Computer Society.

[19] Birger Andersson, Maria Bergholtz, Bertrand Grégoire, Paul Johannesson, Michael Schmitt, and Jelena Zdravkovic. From business to process models - a chaining methodology. In Proceedings of the 8th International Conference on the Advanced Information Systems and Engineering (CAiSE'06), 2006.

[20] Hans Weigand, Paul Johannesson, Birger Andersson, Maria Bergholtz, Ananda Edirisuriya, and Tharaka Ilayperuma. Value object analysis and the transformation from value model to process model. In Enterprise Interoperability, pages 55-65. Springer London, 2007. 
[21] Roel Wieringa, Vincent Pijpers, Lianne Bodenstaff, and Jaap Gordijn. Value-driven coordination process design using physical delivery models. In Qing Li, Stefano Spaccapietra, Eric $\mathrm{Yu}$, and Antoni Oliv'e, editors, Conceptual Modeling - ER 2008, 27th International Conference on Conceptual Modeling, volume 5231 of LNCS, pages 216-231. Springer, 2008.

[22] R. J. Wieringa and J. Gordijn. Value-oriented design of service coordination processes: Correctness and trust. In 20th ACM Symposium on Applied Computing, Santa Fe, New Mexico, USA, pages 1320-1327, New York, NY, USA, March 2005. ACM Press.

[23] Zlatko Zlatev and Andreas Wombacher. Consistency between e3-value models and activity diagrams in a multiperspective development method. In Robert Meersman, Zahir Tari, Mohand-Said Hacid, John Mylopoulos, Barbara Pernici, Özalp Babaoglu, Hans-Arno Jacobsen, Joseph P. Loyall, Michael Kifer, and Stefano Spaccapietra, editors, OTM Conferences (1), volume 3760 of Lecture Notes in Computer Science, pages 520-538. Springer, 2005.

[24] Vincent Pijpers and Jaap Gordijn. Consistency checking between value models and process models: A best-of-breed approach. In Proceedings of the Third International Workshop on Business/IT Alignment and Interoperability (BUSITAL'08) held in conjunction with CAiSE'08 Conference, pages 58-72. CEUR-WS.org, 2008.

[25] L. Bodenstaff, A. Wombacher, and M. U. Reichert. Dynamic consistency between value and coordination models - research issues. Technical Report TR-CTIT-06-50, Enschede, 2006.

[26] L. Bodenstaff, A. Wombacher, and M. U. Reichert. On formal consistency between value and coordination models. Technical Report TR-CTIT-07-91, Enschede, October 2007.

[27] Jaap Gordijn. Value-based requirements Engineering: Exploring innovatie e-commerce ideas. PhD thesis, Vrije Universiteit Amsterdam, 2002.

[28] Birgit Hofreiter and Christian Huemer. A model-driven topdown approach to inter-organizational systems: From global choreography models to executable BPEL. In Proceedings of the 10th IEEE Conference on E-Commerce Technology 
and the Fifth IEEE Conference on Enterprise Computing, ECommerce and E-Services, pages 136-145, Washington, DC, USA, 2008. IEEE Computer Society.

[29] UN/CEFACT. UN/CEFACT Modeling Methodology (UMM), UMM Meta Model - Foundation Module, Mar. 2006. Technical Specification V1.0, http://www.unece.org/cefact/umm/umm_index.htm.

[30] Theodore L. Turocy and Bernhard von Stengel. Game theory. In Encyclopedia of Information Systems, Volume 2. San Diego: Elsevier Science, pages 403-420, 2002.

[31] M. J. Osborne and A. Rubenstein. A course in game theory. Cambridge, Massachusetts: The MIT Press, 1994.

[32] David A. Easley and Jon M. Kleinberg. Networks, Crowds, and Markets - Reasoning About a Highly Connected World. Cambridge University Press, 2010.

[33] Roel Wieringa. Design Methods for Reactive Systems: Yourdon, Statemate and the UML. Morgan Kaufmann Publishers, 2003.

[34] Roel Wieringa, Neil Maiden, Nancy Mead, and Colette Rolland. Requirements engineering paper classification and evaluation criteria: a proposal and a discussion. Requirements Engineering, 11(1):102-107, 2006.

[35] Roel Wieringa. Design science as nested problem solving. In Proceedings of the 4th International Conference on Design Science Research in Information Systems and Technology, DESRIST '09, pages 8:1-8:12, New York, NY, USA, 2009. ACM.

[36] Jaap Gordijn and Hans Akkermans. $e^{3}$ value: Design and evaluation of e-business models. IEEE Intelligent Systems, 16(4):11-17, 2001.

[37] Jaap Gordijn, Eric Yu, and Bas van der Raadt. e-service design using $i^{*}$ and $e^{3}$ value modeling. IEEE Software, 23:26$33,2006$.

[38] M.B. Holbrook. Consumer value: a framework for analysis and research. Routledge interpretive marketing research series. Routledge, 1999. 
[39] Ernst Fehr and Simon Gächter. Fairness and retaliation: The economics of reciprocity. Journal of Economic Perspectives, 14:159-181, 2000.

[40] Vera Kartseva. Designing Controls for Network Organization: A Value-Based Approach. Phd thesis, Vrije Universiteit Amsterdam, October 2007.

[41] Yao hua Tan and Walter Thoen. Formal aspects of a generic model of trust for electronic commerce. In Journal of Decision Support Systems, pages 233-246, 2002.

[42] Mathias Weske. Business Process Management: Concepts, Languages, Architectures. Springer, 2007.

[43] Object Management Group (OMG). Business Process Model and Notation (BPMN), Version 2.0. January 2011.

[44] Gero Decker, Remco Dijkman, Marlon Dumas, and Luciano García-Bañuelos. The business process modeling notation. In Modern Business Process Automation, pages 347-368, 2010.

[45] Martin Fowler. UML Distilled: A Brief Guide to the Standard Object Modeling Language, 3rd Edition. Addison-Wesley, 2004.

[46] Cedric G. Tyler and Stephen R. Baker. Business Genetics. John Wiley and Sons, 2007.

[47] Bart-Jan Hommes. The Evaluation of Business Process Modeling Techniques. PhD thesis, Delft University of Technology, 2004 .

[48] Extensible Markup Language (XML) 1.0 (Fifth Edition) - W3C Recommendation 26 November 2008. available at: http://www.w3.org/TR/REC-xml/.

[49] Web Services Choreography Description Language (WSCDL), Version 1.0 - W3C candidate recommendation 9 november 2005. available at: http://www.w3.org/TR/ws-cdl-10/.

[50] Roger B. Myerson. Game Theory: Analysis of Conflict. Harvard University Press, 1991.

[51] Hassan Fatemi, Marten van Sinderen, and R. J. Wieringa. Value-oriented coordination process modeling. 8th International Conference on Business Process Management (BPM 2010), 6336/2010:162-177, September 2010. 
[52] Hassan Fatemi, Marten van Sinderen, and R. J. Wieringa. From business value model to coordination process model. In Proceedings of the Second IFIP WG5.8 International Workshop on Enterprise Interoperability (IWEI 2009), Valencia, Spain, volume 38 of Lecture Notes in Business Information Processing 38, pages 94-106. Springer, 2009.

[53] Hassan Fatemi, Marten van Sinderen, and R. J. Wieringa. Value-oriented coordination process model engineering. In 6th International Workshop on BUSinness/IT ALignment and Interoperability (BUSITAL 2011), volume 83 of Lecture Notes in Business Information Processing 83, pages 41-44. Springer Verlag, June 2011.

[54] L. Bodenstaff, A. Wombacher, R.J. Wieringa, and M.U. Reichert. An approach for maintaining models of an e-commerce collaboration. In 5th IEEE Conference on Enterprise Computing, E-Commerce, and E-Services, CEC/EEE 2008, pages 239-246, Los Alamitos, July 2008. IEEE Computer Society Press.

[55] Hassan Fatemi, Marten van Sinderen, and R. J. Wieringa. Value to bpmn model transformation. In 12th IFIP Working Conference on Virtual Enterprises (PRO-VE' 11), Adaptation and Value Creating Collaborative Networks, volume 362 of IFIP Advances in Information and Communication Technology 362, pages 333-340. Springer Boston, 2011.

[56] Amir Ghamarian, Maarten de Mol, Arend Rensink, Eduardo Zambon, and Maria Zimakova. Modelling and analysis using GROOVE. International Journal on Software Tools for Technology Transfer (STTT), pages 1-26, 2011.

[57] Krzysztof Czarnecki and Simon Helsen. Classification of model transformation approaches. OOPSLA'03 Workshop on Generative Techniques in the Context of Model-Driven Architecture, 2003.

[58] Andreas Metzger. A Systematic Look at Model Transformations. In Model-Driven Software Development, pages 19-33. Springer Berlin Heidelberg, Berlin/Heidelberg, 2005.

[59] Tom Mens, Krzysztof Czarnecki, and Pieter Van Gorp. A taxonomy of model transformation. In Proc. Dagstuhl Seminar on "Language Engineering for Model-Driven Software Development". Internationales Begegnungs- und Forschungszentrum (IBFI), Schloss Dagstuhl. Electronic, 2005. 
[60] Hassan Fatemi, Marten van Sinderen, and R. J. Wieringa. Trust and business webs. 15th IEEE International EDOC Conference (EDOC 2011), pages 114-121, 29th August - 2nd September 2011.

[61] Hassan Fatemi, Marten van Sinderen, and R. J. Wieringa. A trust ontology for business collaborations. In The 5th IFIP WG8.1 Working Conference on the Practice of Enterprise Modelling (PoEM). November 7th - 8th, 2012; Rostock, Germany, 2012.

[62] Niklas Luhmann. Trust and Power. John Wiley and Sons Ltd., 1979.

[63] Kenneth J. Arrow. Information and economic behavior. Federation of Swedish Industries, Stockholm, Sweden, 1973.

[64] D. Harrison McKnight and Norman L. Chervany. The meanings of trust. Scientific report, Carlson School of Management, University of Minnesota, 1996.

[65] Piotr Sztompka. Trust: A Sociological Theory. Cambridge University Press, 2006.

[66] Barbara Misztal. Trust in Modern Societies: The Search for the Bases of Social Order. Polity Press, 1996.

[67] Annette Baier. Trust and Antitrust. in Ethics, 96(2):231-260, 1986.

[68] R.J. Lewicki and B. B. Bunker. Trust in relationships: A model of trust development and decline. in Conflict, Cooperation and Justice, pages 133-173, 1995.

[69] Jerry Evensky. Adam smith's theory of moral sentiments: On morals and why they matter to a liberal society of free people and free markets. Journal of Economic Perspectives, 19(3-Summer):109-130, 2005.

[70] Francis Fukuyama. Trust: The Social Virtues and the Creation of Prosperity. Free Press, New York, first edition, 1995.

[71] Douglas A. Houston. Trust in the networked economy: Doing business on web time. Business Horizons, 44(2):38-44, March-April 2001.

[72] Sarvapali D. Ramchurn, Dong Huynh, and Nicholas R. Jennings. Trust in multi-agent systems. The Knowledge Engineering Review, 19(1):1-25, 2004. 
[73] D. Harrison Mcknight and Norman L. Chervany. What trust means in e-commerce customer relationships: An interdisciplinary conceptual typology. International Journal of Electronic Commerce, 6:35-59, 2002.

[74] ITU-T Recommendation X.509 (2008), ISO/IEC 9594-8: Information Technology - Open Systems Interconnection - The Directory: Public-Key and Attribute Certificate Frameworks.

[75] Alex C. Michalos. The impact of trust on business, international security and the quality of life. Journal of Business Ethics, 9(8):619-638, 1990.

[76] Cynthia Johnson-Georgea and Walter C. Swap. Measurement of specific interpersonal trust: Construction and validation of a scale to assess trust in a specific other. Journal of Personality and Social Psychology, 43(6):1306-1317, December 1982.

[77] William E. McCarthy. The REA Accounting Model: A Generalized Framework for Accounting Systems in a Shared Data Environment. Accounting Review, 57(3), 1982.

[78] Guido L. Geerts and William E. McCarthy. An accounting object infrastructure for knowledge-based enterprise models. IEEE Intelligent Systems, 14(4):89-94, July 1999.

[79] Alexander Osterwalder. The Business Model Ontology - a proposition in a design science approach. 2004.

[80] Michael Genesereth and Nils Nilsson. Logical Foundations of Artificial Intelligence. Morgan Kaufmann, 1987.

[81] Hans Akkermans and Jaap Gordijn. Ontology engineering, scientific method and the research agenda. In Proceedings of the 15th international conference on Managing Knowledge in a World of Networks, EKAW'06, pages 112-125, Berlin, Heidelberg, 2006. Springer-Verlag.

[82] Ziv Baida, Jaap Gordijn, Hans Akkermans, De Boelelaan A, Andrei Z. Morch, and Hanne Sæle. Ontology-based analysis of e-service bundles for networked enterprises. In In Proceedings of The 17th Bled eCommerce Conference (Bled 2004),

[83] Birger Andersson, Maria Bergholtz, Ananda Edirisuriya, Tharaka Ilayperuma, Paul Johannesson, Jaap Gordijn, Bertrand Grégoire, Michael Schmitt, Eric Dubois, Sven Abels, Axel Hahn, Benkt Wangler, and Hans Weigand. Towards a reference ontology for business models. volume 4215 of Lecture 
Notes in Computer Science, pages 482-496. Springer Berlin / Heidelberg, 2006.

[84] Elizabeth Chang, Tharam S. Dillon, and Farookh Hussain. Trust ontologies for e-service environments: Research articles. International Journal of Intelligent Systems, 22(5):519-545, May 2007.

[85] Stefan Schmidt, Tharam S. Dillon, Robert Steele, and Elizabeth Chang. Trust and reputation ontologies for electronic business. In ICEIS (3), pages 308-315, 2007.

[86] Jingwei Huang and Mark S. Fox. An ontology of trust: formal semantics and transitivity. In ICEC, volume 156 of ACM International Conference Proceeding Series, pages 259-270. ACM, 2006 .

[87] Lea Viljanen. Towards an ontology of trust. In Proceedings of the Second international conference on Trust, Privacy, and Security in Digital Business, TrustBus'05, pages 175-184. Springer-Verlag/ Berlin, Heidelberg, 2005.

[88] Howard S. Becker. Tricks of the Trade : How to Think about Your Research While You're Doing It (Chicago Guides to Writing, Editing, and Publishing). University Of Chicago Press, January 1998.

[89] Jaap Gordijn and Yao-Hua Tan. A design methodology for modeling trustworthy value webs. In International Journal of Electronic Commerce, volume 9, pages 31-48, 2005.

[90] Audun Jøsang and Roslan Ismail. The beta reputation system. In Proceedings of the 15th Bled Electronic Commerce Conference, pages 324-337, June 17 - 192002.

[91] W. T. Luke Teacy, Jigar Patel, Nicholas R. Jennings, and Michael Luck. Coping with inaccurate reputation sources: Experimental analysis of a probabilistic trust model. In AAMAS '05: Proceedings of the fourth international joint conference on Autonomous agents and multiagent systems, pages 997-1004, July 2005.

[92] Thomas Tran and Robin Cohen. Improving user satisfaction in agent-based electronic marketplaces by reputation modelling and adjustable product quality. AAMAS '04: Proceedings of the Third International Joint Conference on $\mathrm{Au}-$ tonomous Agents and Multiagent Systems, pages 828-835, 2004 . 
[93] Henner Gimpel Christof Weinhardt Till J. Winkler, Jochen Haller. Trust indicator modeling for a reputation service in virtual organizations. In: The 15th European Conference on Information Systems (ECIS), 2006.

[94] Jie Zhang. Promoting Honesty in Electronic Marketplaces: Combining Trust Modeling and Incentive Mechanism Design. PhD thesis, Computer Science Department, University of Waterloo, Ontario, Canada, 2009.

[95] Tyrone Grandison and Morris Sloman. A survey of trust in internet applications. IEEE Communications Surveys and Tutorials, 3(4), 2000.

[96] Sini Ruohomaa and Lea Kutvonen. Trust management survey. Proceedings of Third International Conference, iTrust 2005, Lecture Notes in Computer Science, pages 77-92, 2005.

[97] Audun Jøsang, Roslan Ismail, and Colin Boyd. A survey of trust and reputation systems for online service provision. Decision Support Systems, 43(2):618-644, 2007.

[98] Diego Gambetta. Can we trust trust? Trust: Making and Breaking Cooperative Relations, pages 213-237, 1988.

[99] Jochen Haller. A stochastic approach for trust management. Proceedings of the 22nd International Conference on Data Engineering (ICDE) Workshops, 3-7 April 2006.

[100] Jeffrey H. Dyer and Wujin Chu. The role of trustworthiness in reducing transaction costs and improving performance: Empirical evidence from the United States, Japan, and Korea. Organization Science, 14(1):57-68, January-February 2003.

[101] Peter Hwang and Willem P. Burgers. Properties of trust: An analytical view. Organizational Behavior and Human Decision Processes, 69:67-73, January 1997.

[102] Hassan Fatemi, Marten van Sinderen, and R. J. Wieringa. Managing trust in business webs using game theory. In The 26th IEEE International Conference on Advanced Information Networking and Applications (AINA-2012), The International Workshop on Networked Virtual Enterprises (NetVE 2012), IEEE Computer Society. 2012.

[103] Michael Whinston Andreu Mas-Collel and Jerry Green. Microeconomic Theory. Oxford University Press, 1995. 
[104] Leonard Savage. The Foundations of Statistics. Wiley, 1954.

[105] Hal Varian. Intermediate Microeconomics: A Modern Approach. Norton, 2003.

[106] John F. Nash. Equilibrium points in n-person games. Proceedings of the National Academy of Sciences of the United States of America, 36(1):48-49, January 1950.

[107] John Nash. Non-cooperative games. The Annals of Mathematics, 54(2):286-295, 1951.

[108] Hassan Fatemi, Marten van Sinderen, R. J. Wieringa, and Ivan S. Razo-Zapata. Endurability and profitability analysis of collaborative networks. In 25th Bled eConference. 2012.

[109] Ivan Razo-Zapata, Pieter De Leenheer, and Hans Akkermans. Service value networks for competency-driven educational services: A case study. In Proceedings of the 6th international BUSITAL workshop, 2011. 
REFERENCES 


\section{Author Publications}

Hassan Fatemi, Marten van Sinderen, and R. J. Wieringa. Managing trust in business webs using game theory. In The 26th IEEE International Conference on Advanced Information Networking and Applications (AINA-2012), The International Workshop on Networked Virtual Enterprises (NetVE 2012), IEEE Computer Society. 2012.

Hassan Fatemi, Marten van Sinderen, and R. J. Wieringa. A trust ontology for business collaborations. In The 5th IFIP WG8.1 Working Conference on the Practice of Enterprise Modelling (PoEM). November 7th - 8th, 2012; Rostock, Germany, 2012.

Hassan Fatemi, Marten van Sinderen, R. J. Wieringa, and Ivan S. Razo-Zapata. Endurability and profitability analysis of collaborative networks. In 25th Bled eConference. 2012.

Hassan Fatemi, Marten van Sinderen, and R. J. Wieringa. Valueoriented coordination process model engineering. In 6th International Workshop on BUSinness/IT ALignment and Interoperability (BUSITAL 2011), volume 83 of Lecture Notes in Business Information Processing 83, pages 41-44. Springer Verlag, June 2011.

Hassan Fatemi, Marten van Sinderen, and R. J. Wieringa. Trust and business webs. 15th IEEE International EDOC Conference (EDOC 2011), pages 114-121, 29th August - 2nd September 2011.

Hassan Fatemi, Marten van Sinderen, and R. J. Wieringa. Value to bpmn model transformation. In 12th IFIP Working Conference on Virtual Enterprises (PRO-VE' 11), Adaptation and Value Creating Collaborative Networks, volume 362 of IFIP Advances in Information and Communication Technology 362, pages 333-340. Springer Boston, 2011. 
Hassan Fatemi, Marten van Sinderen, and R. J. Wieringa. Value-oriented coordination process modeling. 8th International Conference on Business Process Management (BPM 2010), 6336/2010:162-177, September 2010.

Hassan Fatemi, Marten van Sinderen, and R. J. Wieringa. From business value model to coordination process model. In Proceedings of the Second IFIP WG5.8 International Workshop on Enterprise Interoperability (IWEI 2009), Valencia, Spain, volume 38 of Lecture Notes in Business Information Processing 38, pages 94-106. Springer, 2009. 


\section{SIKS Dissertation Series}

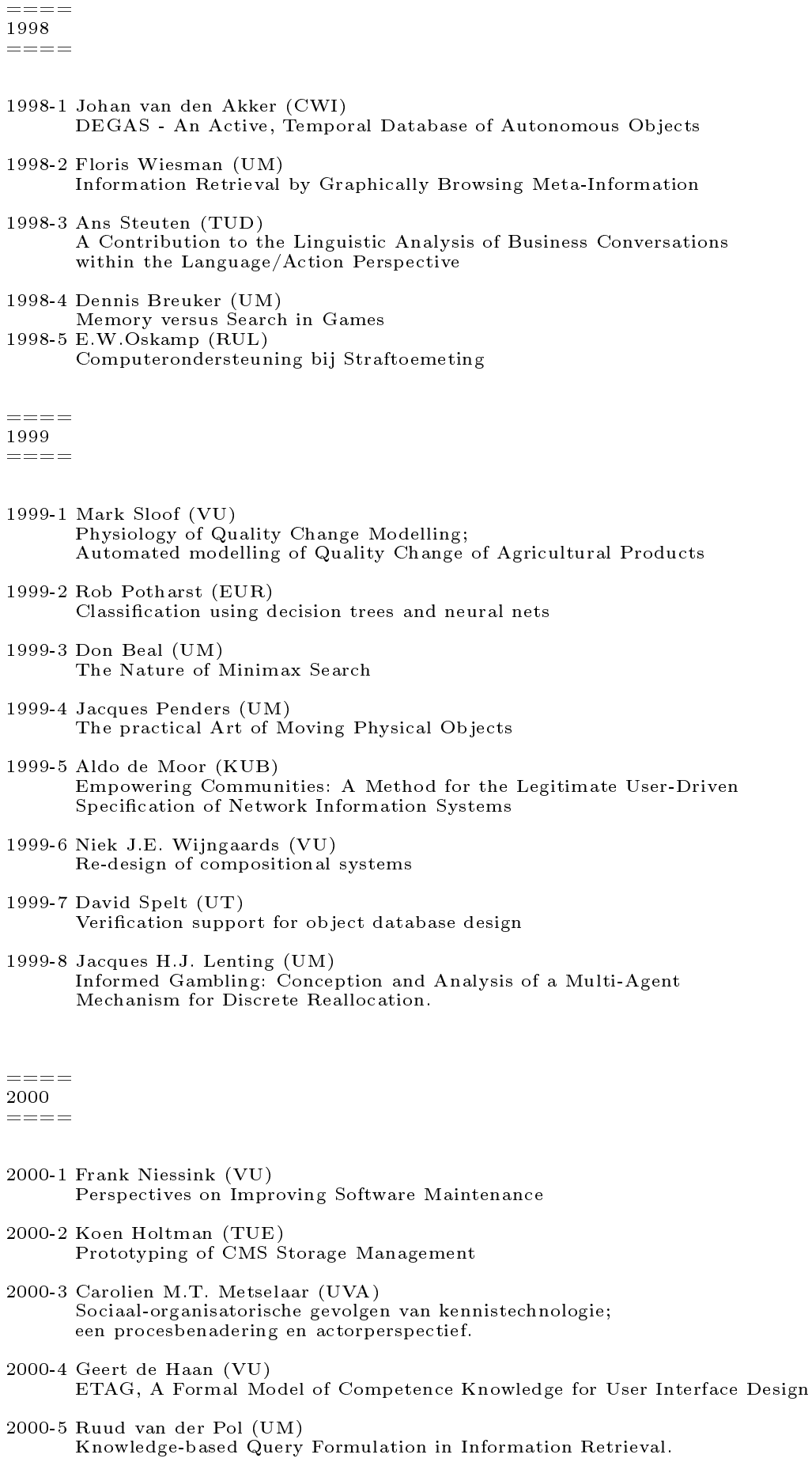




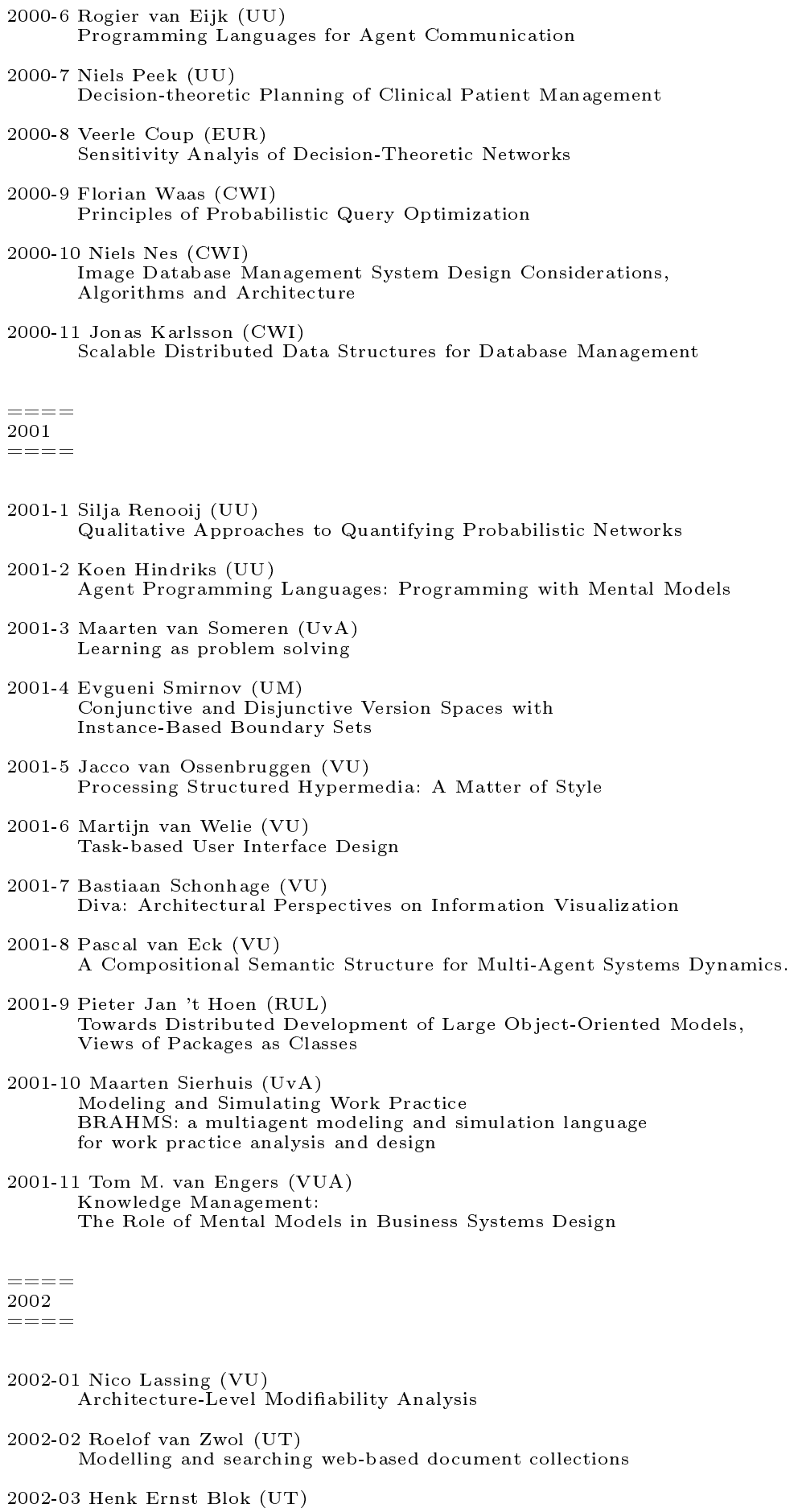


Database Optimization Aspects for Information Retrieval

2002-04 Juan Roberto Castelo Valdueza (UU)

The Discrete Acyclic Digraph Markov Model in Data Mining

2002-05 Radu Serban (VU)

The Private Cyberspace Modeling Electronic Environments

inhabited by Privacy-concerned Agents

2002-06 Laurens Mommers (UL)

Applied legal epistemology;

Building a knowledge-based ontology of the legal domain

2002-07 Peter Boncz (CWI)

Monet: A Next-Generation DBMS Kernel For Query-Intensive Applications

2002-08 Jaap Gordijn (VU)

Value Based Requirements Engineering: Exploring Innovative

E-Commerce Ideas

2002-09 Willem-Jan van den Heuvel(KUB)

Integrating Modern Business Applications with Objectified Legacy Systems

2002-10 Brian Sheppard (UM)

Towards Perfect Play of Scrabble

2002-11 Wouter C.A. Wijngaards (VU)

Agent Based Modelling of Dynamics: Biological and Organisational Applications

2002-12 Albrecht Schmidt (Uva)

Processing XML in Database Systems

2002-13 Hongjing Wu (TUE)

A Reference Architecture for Adaptive Hypermedia Applications

2002-14 Wieke de Vries (UU)

Agent Interaction: Abstract Approaches to Modelling, Programming and

Verifying Multi-Agent Systems

2002-15 Rik Eshuis (UT)

Semantics and Verification of UML Activity Diagrams for Workflow Modelling

2002-16 Pieter van Langen (VU)

The Anatomy of Design: Foundations, Models and Applications

2002-17 Stefan Manegold (UVA)

Understanding, Modeling, and Improving Main-Memory Database Performance

2003

2003-01 Heiner Stuckenschmidt (VU)

Ontology-Based Information Sharing in Weakly Structured Environments

2003-02 Jan Broersen (VU)

Modal Action Logics for Reasoning About Reactive Systems

2003-03 Martijn Schuemie (TUD)

Human-Computer Interaction and Presence in Virtual Reality Exposure Therapy

2003-04 Milan Petkovic (UT)

Content-Based Video Retrieval Supported by Database Technology

2003-05 Jos Lehmann (UVA)

Causation in Artificial Intelligence and Law - A modelling approach

2003-06 Boris van Schooten (UT)

Development and specification of virtual environments

2003-07 Machiel Jansen (UvA)

Formal Explorations of Knowledge Intensive Tasks

2003-08 Yongping Ran (UM)

Repair Based Scheduling 


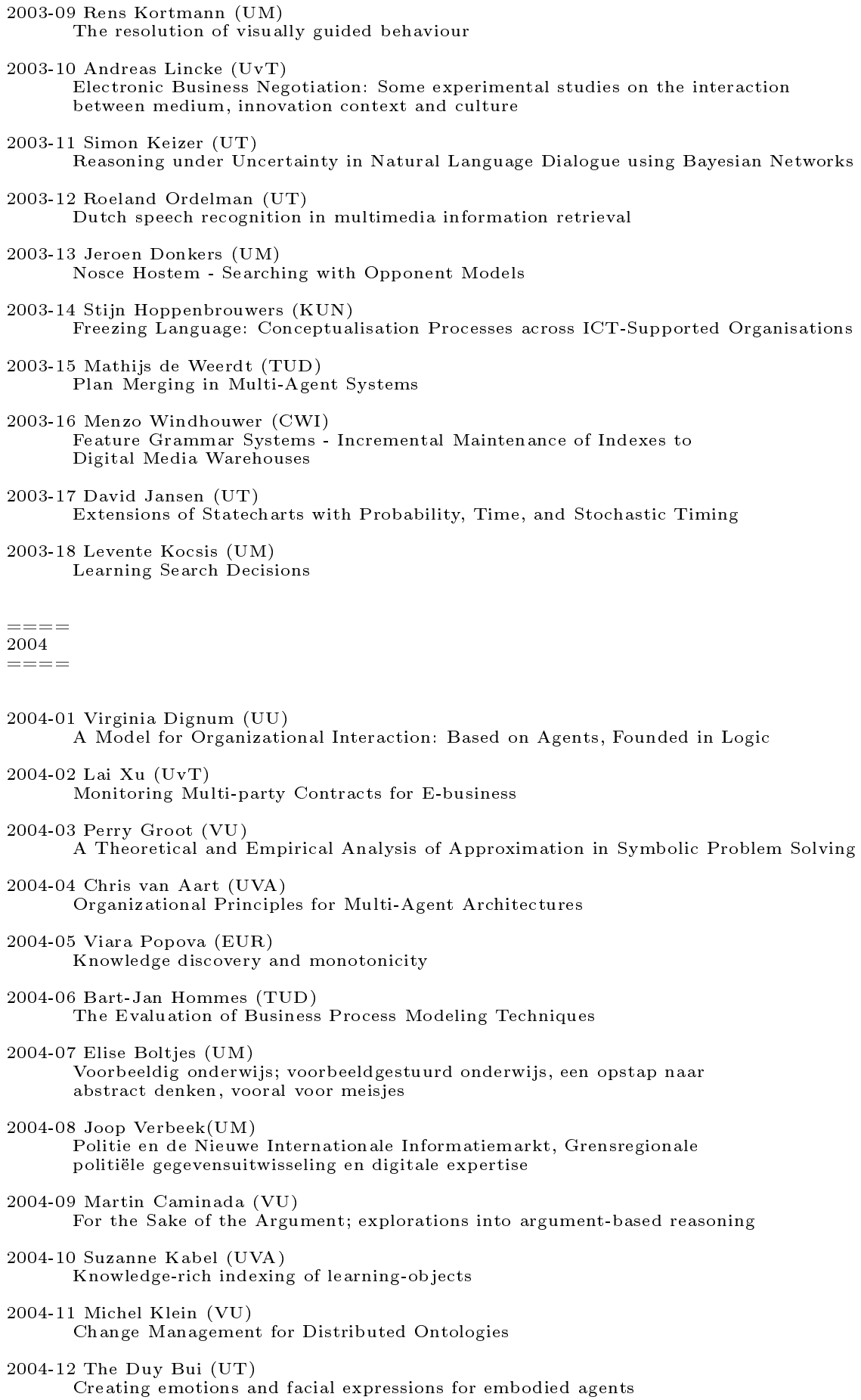


2004-13 Wojciech Jamroga (UT)

Using Multiple Models of Reality: On Agents who Know how to Play

2004-14 Paul Harrenstein (UU)

Logic in Conflict. Logical Explorations in Strategic Equilibrium

2004-15 Arno Knobbe (UU)

Multi-Relational Data Mining

2004-16 Federico Divina (VU)

Hybrid Genetic Relational Search for Inductive Learning

2004-17 Mark Winands (UM)

Informed Search in Complex Games

2004-18 Vania Bessa Machado (UvA)

Supporting the Construction of Qualitative Knowledge Models

2004-19 Thijs Westerveld (UT)

Using generative probabilistic models for multimedia retrieval

2004-20 Madelon Evers (Nyenrode)

Learning from Design: facilitating multidisciplinary design teams

$\overline{\overline{2}}=$

$===$

2005-01 Floor Verdenius (UVA)

Methodological Aspects of Designing Induction-Based Applications

2005-02 Erik van der Werf (UM))

AI techniques for the game of Go

2005-03 Franc Grootjen (RUN)

A Pragmatic Approach to the Conceptualisation of Language

2005-04 Nirvana Meratnia (UT)

Towards Database Support for Moving Object data

2005-05 Gabriel Infante-Lopez (UVA)

Two-Level Probabilistic Grammars for Natural Language Parsing

2005-06 Pieter Spronck (UM)

Adaptive Game AI

2005-07 Flavius Frasincar (TUE)

Hypermedia Presentation Generation for Semantic Web Information Systems

2005-08 Richard Vdovjak (TUE)

A Model-driven Approach for Building Distributed Ontology-based

Web Applications

2005-09 Jeen Broekstra (VU)

Storage, Querying and Inferencing for Semantic Web Languages

2005-10 Anders Bouwer (UVA)

Explaining Behaviour: Using Qualitative Simulation in

Interactive Learning Environments

2005-11 Elth Ogston (VU)

Agent Based Matchmaking and Clustering - A Decentralized Approach to Search

2005-12 Csaba Boer (EUR)

Distributed Simulation in Industry

2005-13 Fred Hamburg (UL)

Een Computermodel voor het Ondersteunen van Euthanasiebeslissingen

2005-14 Borys Omelayenko (VU)

Web-Service configuration on the Semantic Web; Exploring how semantics

meets pragmatics

2005-15 Tibor Bosse (VU) 
Analysis of the Dynamics of Cognitive Processes

2005-16 Joris Graaumans (UU)

Usability of XML Query Languages

2005-17 Boris Shishkov (TUD)

Software Specification Based on Re-usable Business Components

2005-18 Danielle Sent (UU)

Test-selection strategies for probabilistic networks

2005-19 Michel van Dartel (UM)

Situated Representation

2005-20 Cristina Coteanu (UL)

Cyber Consumer Law, State of the Art and Perspectives

2005-21 Wijnand Derks (UT)

Improving Concurrency and Recovery in Database Systems by

Exploiting Application Semantics

$\overline{=}=$

$===$

2006-01 Samuil Angelov (TUE)

Foundations of B2B Electronic Contracting

2006-02 Cristina Chisalita (VU)

Contextual issues in the design and use of information technology in organizations

2006-03 Noor Christoph (UVA)

The role of metacognitive skills in learning to solve problems

2006-04 Marta Sabou (VU)

Building Web Service Ontologies

2006-05 Cees Pierik (UU)

Validation Techniques for Object-Oriented Proof Outlines

2006-06 Ziv Baida (VU)

Software-aided Service Bundling - Intelligent Methods \& Tools

for Graphical Service Modeling

2006-07 Marko Smiljanic (UT)

XML schema matching - balancing efficiency and effectiveness by means of clustering

2006-08 Eelco Herder (UT)

Forward, Back and Home Again - Analyzing User Behavior on the Web

2006-09 Mohamed Wahdan (UM)

Automatic Formulation of the Auditor's Opinion

2006-10 Ronny Siebes (VU)

Semantic Routing in Peer-to-Peer Systems

2006-11 Joeri van Ruth (UT)

Flattening Queries over Nested Data Types

2006-12 Bert Bongers (VU)

Interactivation - Towards an e-cology of people, our technological environment, and the arts

2006-13 Henk-Jan Lebbink (UU)

Dialogue and Decision Games for Information Exchanging Agents

2006-14 Johan Hoorn (VU)

Software Requirements: Update, Upgrade, Redesign - towards a Theory of

Requirements Change

2006-15 Rainer Malik (UU)

CONAN: Text Mining in the Biomedical Domain

2006-16 Carsten Riggelsen (UU)

Approximation Methods for Efficient Learning of Bayesian Networks 
2006-17 Stacey Nagata (UU)

User Assistance for Multitasking with Interruptions on a Mobile Device

2006-18 Valentin Zhizhkun (UVA)

Graph transformation for Natural Language Processing

2006-19 Birna van Riemsdijk (UU)

Cognitive Agent Programming: A Semantic Approach

2006-20 Marina Velikova (UvT)

Monotone models for prediction in data mining

2006-21 Bas van Gils (RUN)

Aptness on the Web

2006-22 Paul de Vrieze (RUN)

Fundaments of Adaptive Personalisation

2006-23 Ion Juvina (UU)

Development of Cognitive Model for Navigating on the Web

2006-24 Laura Hollink (VU)

Semantic Annotation for Retrieval of Visual Resources

2006-25 Madalina Drugan (UU)

Conditional log-likelihood MDL and Evolutionary MCMC

2006-26 Vojkan Mihajlovic (UT)

Score Region Algebra: A Flexible Framework for Structured

Information Retrieval

2006-27 Stefano Bocconi (CWI)

Vox Populi: generating video documentaries from semantically annotated media repositories

2006-28 Borkur Sigurbjornsson (UVA)

Focused Information Access using XML Element Retrieval

$\overline{=}==$

2007-01 Kees Leune (UvT)

Access Control and Service-Oriented Architectures

2007-02 Wouter Teepe (RUG)

Reconciling Information Exchange and Confidentiality: A Formal Approach

2007-03 Peter Mika (VU)

Social Networks and the Semantic Web

2007-04 Jurriaan van Diggelen (UU)

Achieving Semantic Interoperability in Multi-agent Systems:

a dialogue-based approach

2007-05 Bart Schermer (UL)

Software Agents, Surveillance, and the Right to Privacy:

a Legislative Framework for Agent-enabled Surveillance

2007-06 Gilad Mishne (UVA)

Applied Text Analytics for Blogs

2007-07 Natasa Jovanovic' (UT)

To Whom It May Concern - Addressee Identification in Face-to-Face Meetings

2007-08 Mark Hoogendoorn (VU)

Modeling of Change in Multi-Agent Organizations

2007-09 David Mobach (VU)

Agent-Based Mediated Service Negotiation

2007-10 Huib Aldewereld (UU)

Autonomy vs. Conformity: an Institutional Perspective on Norms and Protocols

2007-11 Natalia Stash (TUE) 
Incorporating Cognitive/Learning Styles in a General-Purpose Adaptive Hypermedia System

2007-12 Marcel van Gerven (RUN)

Bayesian Networks for Clinical Decision Support: A Rational Approach

to Dynamic Decision-Making under Uncertainty

2007-13 Rutger Rienks (UT)

Meetings in Smart Environments; Implications of Progressing Technology

2007-14 Niek Bergboer (UM)

Context-Based Image Analysis

2007-15 Joyca Lacroix (UM)

NIM: a Situated Computational Memory Model

2007-16 Davide Grossi (UU)

Designing Invisible Handcuffs. Formal investigations in Institutions and Organizations for Multi-agent Systems

2007-17 Theodore Charitos (UU)

Reasoning with Dynamic Networks in Practice

2007-18 Bart Orriens (UvT)

On the development an management of adaptive business collaborations

2007-19 David Levy (UM)

Intimate relationships with artificial partners

2007-20 Slinger Jansen (UU)

Customer Configuration Updating in a Software Supply Network

2007-21 Karianne Vermaas (UU)

Fast diffusion and broadening use: A research on residential adoption and usage of broadband internet in the Netherlands between 2001 and 2005

2007-22 Zlatko Zlatev (UT)

Goal-oriented design of value and process models from patterns

2007-23 Peter Barna (TUE)

Specification of Application Logic in Web Information Systems

2007-24 Georgina Ramírez Camps (CWI)

Structural Features in XML Retrieval

2007-25 Joost Schalken (VU)

Empirical Investigations in Software Process Improvement

2008

2008-01 Katalin Boer-Sorbán (EUR)

Agent-Based Simulation of Financial Markets: A modular,continuous-time approach

2008-02 Alexei Sharpanskykh (VU)

On Computer-Aided Methods for Modeling and Analysis of Organizations

2008-03 Vera Hollink (UVA)

Optimizing hierarchical menus: a usage-based approach

2008-04 Ander de Keijzer (UT)

Management of Uncertain Data - towards unattended integration

2008-05 Bela Mutschler (UT)

Modeling and simulating causal dependencies on process-aware information systems

from a cost perspective

2008-06 Arjen Hommersom (RUN)

On the Application of Formal Methods to Clinical Guidelines,

an Artificial Intelligence Perspective

2008-07 Peter van Rosmalen (OU)

Supporting the tutor in the design and support of adaptive e-learning 


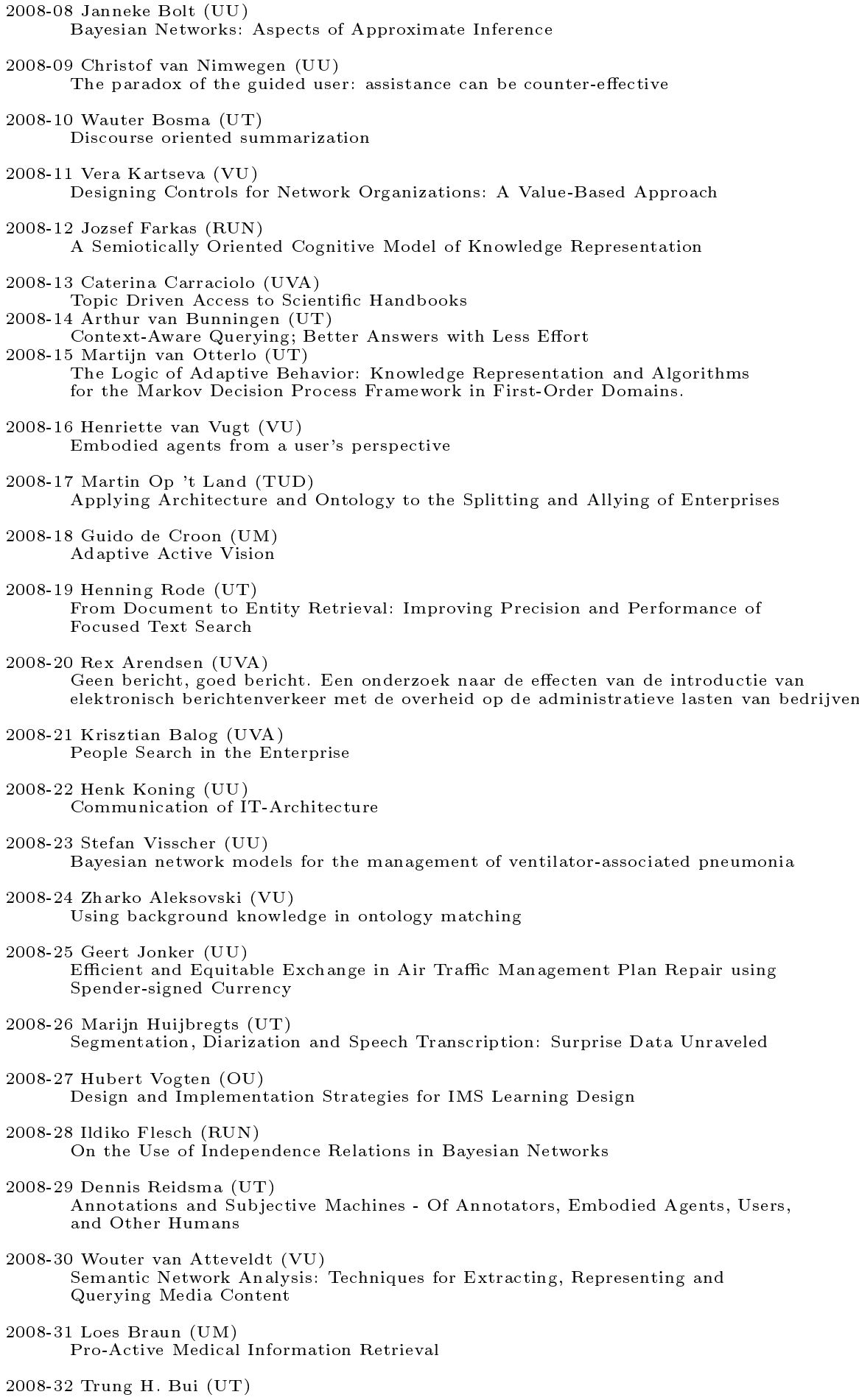


Toward Affective Dialogue Management using Partially Observable Markov Decision Processes

2008-33 Frank Terpstra (UVA)

Scientific Workflow Design; theoretical and practical issues

2008-34 Jeroen de Knijf (UU)

Studies in Frequent Tree Mining

2008-35 Ben Torben Nielsen (UvT)

Dendritic morphologies: function shapes structure

2009

$===$

2009-01 Rasa Jurgelenaite (RUN)

Symmetric Causal Independence Models

2009-02 Willem Robert van Hage (VU)

Evaluating Ontology-Alignment Techniques

2009-03 Hans Stol (UvT)

A Framework for Evidence-based Policy Making Using IT

2009-04 Josephine Nabukenya (RUN)

Improving the Quality of Organisational Policy Making using Collaboration Engineering

2009-05 Sietse Overbeek (RUN)

Bridging Supply and Demand for Knowledge Intensive Tasks -

Based on Knowledge, Cognition, and Quality

2009-06 Muhammad Subianto (UU)

Understanding Classification

2009-07 Ronald Poppe (UT)

Discriminative Vision-Based Recovery and Recognition of Human Motion

2009-08 Volker Nannen (VU)

Evolutionary Agent-Based Policy Analysis in Dynamic Environments

2009-09 Benjamin Kanagwa (RUN)

Design, Discovery and Construction of Service-oriented Systems

2009-10 Jan Wielemaker (UVA)

Logic programming for knowledge-intensive interactive applications

2009-11 Alexander Boer (UVA)

Legal Theory, Sources of Law \& the Semantic Web

2009-12 Peter Massuthe (TUE, Humboldt-Universitaet zu Berlin)

perating Guidelines for Services

2009-13 Steven de Jong (UM)

Fairness in Multi-Agent Systems

2009-14 Maksym Korotkiy (VU)

From ontology-enabled services to service-enabled ontologies

(making ontologies work in e-science with ONTO-SOA)

2009-15 Rinke Hoekstra (UVA)

Ontology Representation - Design Patterns and Ontologies that Make Sense 2009-16 Fritz Reul (UvT)

New Architectures in Computer Chess

2009-17 Laurens van der Maaten (UvT)

Feature Extraction from Visual Data

2009-18 Fabian Groffen (CWI)

Armada, An Evolving Database System

2009-19 Valentin Robu (CWI)

Modeling Preferences, Strategic Reasoning and Collaboration in

Agent-Mediated Electronic Markets

2009-20 Bob van der Vecht (UU) 
Adjustable Autonomy: Controling Influences on Decision Making

2009-21 Stijn Vanderlooy (UM)

Ranking and Reliable Classification

2009-22 Pavel Serdyukov (UT)

Search For Expertise: Going beyond direct evidence

2009-23 Peter Hofgesang (VU)

Modelling Web Usage in a Changing Environment

2009-24 Annerieke Heuvelink (VUA)

Cognitive Models for Training Simulations

2009-25 Alex van Ballegooij (CWI)

"RAM: Array Database Management through Relational Mapping"

2009-26 Fernando Koch (UU)

An Agent-Based Model for the Development of Intelligent Mobile Services

2009-27 Christian Glahn (OU)

Contextual Support of social Engagement and Reflection on the Web

2009-28 Sander Evers (UT)

Sensor Data Management with Probabilistic Models

2009-29 Stanislav Pokraev (UT)

Model-Driven Semantic Integration of Service-Oriented Applications

2009-30 Marcin Zukowski (CWI)

Balancing vectorized query execution with bandwidth-optimized storage

2009-31 Sofiya Katrenko (UVA)

A Closer Look at Learning Relations from Text

2009-32 Rik Farenhorst (VU) and Remco de Boer (VU)

Architectural Knowledge Management: Supporting Architects and Auditors

2009-33 Khiet Truong (UT)

How Does Real Affect Affect Affect Recognition In Speech?

2009-34 Inge van de Weerd (UU)

Advancing in Software Product Management:

An Incremental Method Engineering Approach

2009-35 Wouter Koelewijn (UL)

Privacy en Politiegegevens; Over geautomatiseerde normatieve informatie-uitwisseling

2009-36 Marco Kalz (OUN)

Placement Support for Learners in Learning Networks

2009-37 Hendrik Drachsler (OUN)

Navigation Support for Learners in Informal Learning Networks

2009-38 Riina Vuorikari (OU)

Tags and self-organisation: a metadata ecology for learning resources in

a multilingual context

2009-39 Christian Stahl (TUE, Humboldt-Universitaet zu Berlin)

Service Substitution - A Behavioral Approach Based on Petri Nets

2009-40 Stephan Raaijmakers (UvT)

Multinomial Language Learning: Investigations into the Geometry of Language

2009-41 Igor Berezhnyy (UvT)

Digital Analysis of Paintings

2009-42 Toine Bogers

Recommender Systems for Social Bookmarking

2009-43 Virginia Nunes Leal Franqueira (UT)

Finding Multi-step Attacks in Computer Networks using Heuristic Search and

Mobile Ambients

2009-44 Roberto Santana Tapia (UT)

Assessing Business-IT Alignment in Networked Organizations 
2009-45 Jilles Vreeken (UU)

Making Pattern Mining Useful

2009-46 Loredana Afanasiev (UvA)

Querying XML: Benchmarks and Recursion

$\overline{2010}$

$==-$

2010-01 Matthijs van Leeuwen (UU)

Patterns that Matter

2010-02 Ingo Wassink (UT)

Work flows in Life Science

2010-03 Joost Geurts (CWI)

A Document Engineering Model and Processing Framework for Multimedia documents 2010-04 Olga Kulyk (UT)

Do You Know What I Know? Situational Awareness of Co-located Teams in Multidisplay Environments

2010-05 Claudia Hauff (UT)

Predicting the Effectiveness of Queries and Retrieval Systems

2010-06 Sander Bakkes (UvT)

Rapid Adaptation of Video Game AI

2010-07 Wim Fikkert (UT)

Gesture interaction at a Distance

2010-08 Krzysztof Siewicz (UL)

Towards an Improved Regulatory Framework of Free Software. Protecting user freedoms in a world of software communities and eGovernments

2010-09 Hugo Kielman (UL)

A Politiele gegevensverwerking en Privacy, Naar een effectieve waarborging

2010-10 Rebecca Ong (UL)

Mobile Communication and Protection of Children 2010-11 Adriaan Ter Mors (TUD)

The world according to MARP: Multi-Agent Route Planning

2010-12 Susan van den Braak (UU)

Sensemaking software for crime analysis

2010-13 Gianluigi Folino (RUN)

High Performance Data Mining using Bio-inspired techniques

2010-14 Sander van Splunter (VU)

Automated Web Service Reconfiguration

2010-15 Lianne Bodenstaff (UT)

Managing Dependency Relations in Inter-Organizational Models

2010-16 Sicco Verwer (TUD)

Efficient Identification of Timed Automata, theory and practice

2010-17 Spyros Kotoulas (VU)

Scalable Discovery of Networked Resources: Algorithms, Infrastructure, Applications

2010-18 Charlotte Gerritsen (VU)

Caught in the Act: Investigating Crime by Agent-Based Simulation

2010-19 Henriette Cramer (UvA)

People's Responses to Autonomous and Adaptive Systems

2010-20 Ivo Swartjes (UT)

Whose Story Is It Anyway? How Improv Informs Agency and Authorship of

Emergent Narrative

2010-21 Harold van Heerde (UT)

Privacy-aware data management by means of data degradation

2010-22 Michiel Hildebrand (CWI) 
End-user Support for Access to

Heterogeneous Linked Data

2010-23 Bas Steunebrink (UU)

The Logical Structure of Emotions

2010-24 Dmytro Tykhonov

Designing Generic and Efficient Negotiation Strategies

2010-25 Zulfiqar Ali Memon (VU)

Modelling Human-Awareness for Ambient Agents: A Human Mindreading Perspective

2010-26 Ying Zhang (CWI)

XRPC: Efficient Distributed Query Processing on Heterogeneous XQuery Engines

2010-27 Marten Voulon (UL)

Automatisch contracteren

2010-28 Arne Koopman (UU)

Characteristic Relational Patterns

2010-29 Stratos Idreos(CWI)

Database Cracking: Towards Auto-tuning Database Kernels

2010-30 Marieke van Erp (UvT)

Accessing Natural History - Discoveries in data cleaning, structuring, and retrieval

2010-31 Victor de Boer (UVA)

Ontology Enrichment from Heterogeneous Sources on the Web

2010-32 Marcel Hiel (UvT)

An Adaptive Service Oriented Architecture: Automatically solving

Interoperability Problems

2010-33 Robin Aly (UT)

Modeling Representation Uncertainty in Concept-Based Multimedia Retrieval

2010-34 Teduh Dirgahayu (UT)

Interaction Design in Service Compositions

2010-35 Dolf Trieschnigg (UT)

Proof of Concept: Concept-based Biomedical Information Retrieval

2010-36 Jose Janssen (OU)

Paving the Way for Lifelong Learning; Facilitating competence development

through a learning path specification

2010-37 Niels Lohmann (TUE)

Correctness of services and their composition

2010-38 Dirk Fahland (TUE)

From Scenarios to components

2010-39 Ghazanfar Farooq Siddiqui (VU)

Integrative modeling of emotions in virtual agents

2010-40 Mark van Assem (VU)

Converting and Integrating Vocabularies for the Semantic Web

2010-41 Guillaume Chaslot (UM)

Monte-Carlo Tree Search

2010-42 Sybren de Kinderen (VU)

Needs-driven service bundling in a multi-supplier setting -

the computational e3-service approach

2010-43 Peter van Kranenburg (UU)

A Computational Approach to Content-Based Retrieval of Folk Song Melodies

2010-44 Pieter Bellekens (TUE)

An Approach towards Context-sensitive and User-adapted Access to Heterogeneous Data Sources, Illustrated in the Television Domain

2010-45 Vasilios Andrikopoulos (UvT)

A theory and model for the evolution of software services

2010-46 Vincent Pijpers (VU)

e3alignment: Exploring Inter-Organizational Business-ICT Alignment 
2010-47 Chen Li (UT)

Mining Process Model Variants: Challenges, Techniques, Examples

2010-48 Milan Lovric (EUR)

Behavioral Finance and Agent-Based Artificial Markets

2010-49 Jahn-Takeshi Saito (UM)

Solving difficult game positions

2010-50 Bouke Huurnink (UVA)

Search in Audiovisual Broadcast Archives

2010-51 Alia Khairia Amin (CWI)

Understanding and supporting information seeking tasks in multiple sources

2010-52 Peter-Paul van Maanen (VU)

Adaptive Support for Human-Computer Teams: Exploring the Use of Cognitive Models of Trust and Attention

2010-53 Edgar Meij (UVA)

Combining Concepts and Language Models for Information Access

2011

2011-01 Botond Cseke (RUN)

Variational Algorithms for Bayesian Inference in Latent Gaussian Models

2011-02 Nick Tinnemeier(UU)

Organizing Agent Organizations. Syntax and Operational Semantics of

an Organization-Oriented Programming Language

2011-03

Jan Martijn van der Werf (TUE)

Compositional Design and Verification of Component-Based Information Systems

2011-04 Hado van Hasselt (UU)

Insights in Reinforcement Learning; Formal analysis and empirical evaluation of temporal-difference learning algorithms

2011-05 Base van der Raadt (VU)

Enterprise Architecture Coming of Age - Increasing the Performance of an Emerging Discipline.

2011-06 Yiwen Wang (TUE)

Semantically-Enhanced Recommendations in Cultural Heritage

2011-07 Yujia Cao (UT)

Multimodal Information Presentation for High Load Human Computer Interaction

2011-08 Nieske Vergunst (UU)

BDI-based Generation of Robust Task-Oriented Dialogues 2011-09 Tim de Jong (OU)

Contextualised Mobile Media for Learning

2011-10 Bart Bogaert (UvT)

Cloud Content Contention

2011-11 Dhaval Vyas (UT)

Designing for Awareness: An Experience-focused HCI Perspective

2011-12 Carmen Bratosin (TUE)

Grid Architecture for Distributed Process Mining

2011-13 Xiaoyu Mao (UvT)

Airport under Control. Multiagent Scheduling for Airport Ground Handling

2011-14 Milan Lovric (EUR)

Behavioral Finance and Agent-Based Artificial Markets

2011-15 Marijn Koolen (UvA)

The Meaning of Structure: the Value of Link Evidence for Information Retrieval

2011-16 Maarten Schadd (UM)

Selective Search in Games of Different Complexity 


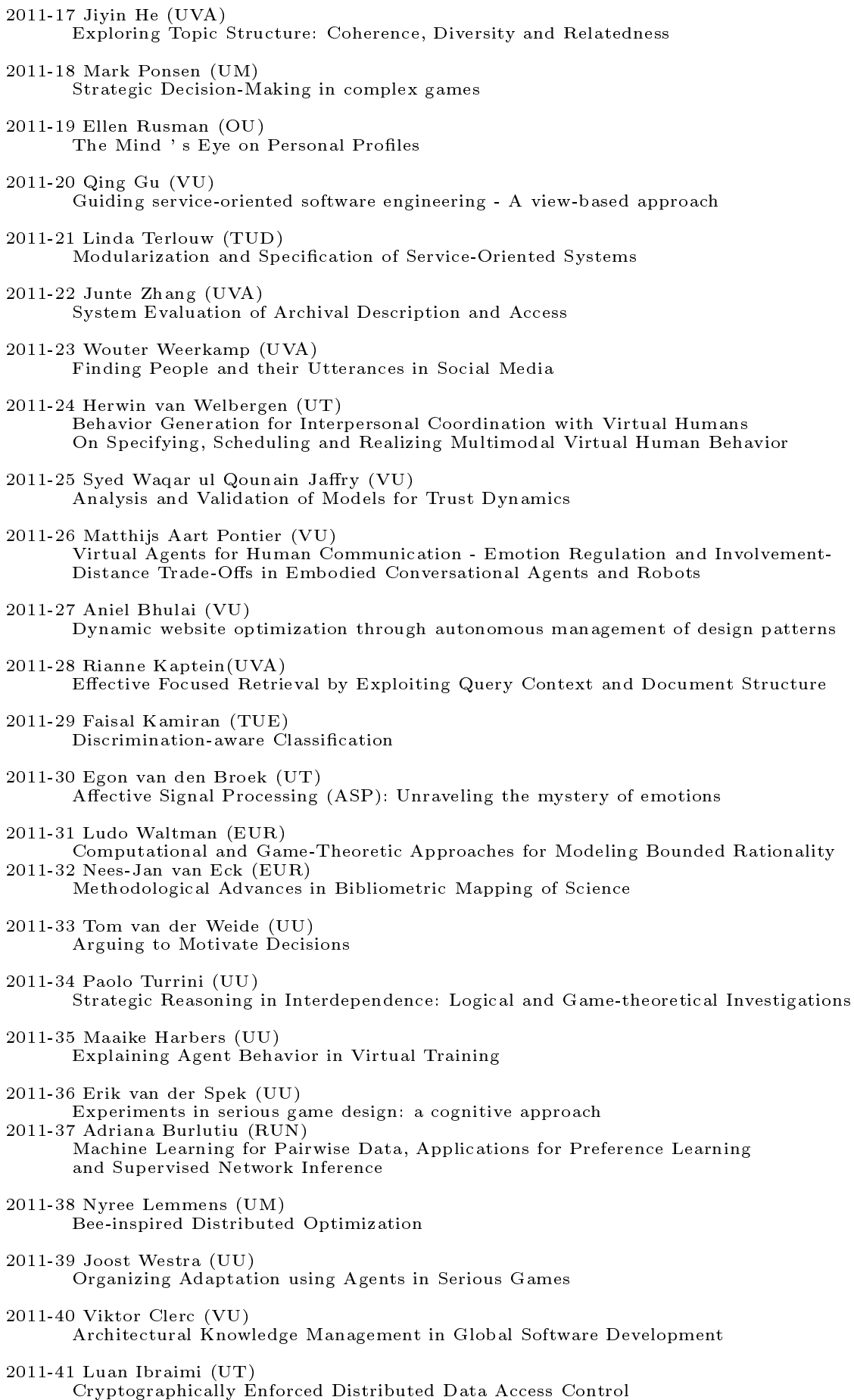


2011-42 Michal Sindlar (UU)

Explaining Behavior through Mental State Attribution

2011-43 Henk van der Schuur (UU)

Process Improvement through Software Operation Knowledge

2011-44 Boris Reuderink (UT)

Robust Brain-Computer Interfaces

2011-45 Herman Stehouwer (UvT)

Statistical Language Models for Alternative Sequence Selection

2011-46 Beibei Hu (TUD)

Towards Contextualized Information Delivery: A Rule-based Architecture

for the Domain of Mobile Police Work

2011-47 Azizi Bin Ab Aziz(VU)

Exploring Computational Models for Intelligent Support of Persons with Depression

2011-48 Mark Ter Maat (UT)

Response Selection and Turn-taking for a Sensitive Artificial Listening Agent

2011-49 Andreea Niculescu (UT)

Conversational interfaces for task-oriented spoken dialogues:

design aspects influencing interaction quality

$===$

2012-01 Terry Kakeeto (UvT)

Relationship Marketing for SMEs in Uganda

2012-02 Muhammad Umair(VU)

Adaptivity, emotion, and Rationality in Human and Ambient Agent Models

2012-03 Adam Vanya (VU)

Supporting Architecture Evolution by Mining Software Repositories

2012-04 Jurriaan Souer (UU)

Development of Content Management System-based Web Applications

2012-05 Marijn Plomp (UU)

Maturing Interorganisational Information Systems

2012-06 Wolfgang Reinhardt (OU)

Awareness Support for Knowledge Workers in Research Networks

2012-07 Rianne van Lambalgen (VU)

When the Going Gets Tough: Exploring Agent-based Models of Human Performance under Demanding Conditions

2012-08 Gerben de Vries (UVA)

Kernel Methods for Vessel Trajectories

2012-09 Ricardo Neisse (UT)

Trust and Privacy Management Support for Context-Aware Service Platforms

2012-10 David Smits (TUE)

Towards a Generic Distributed Adaptive Hypermedia Environment

2012-11 J.C.B. Rantham Prabhakara (TUE)

Process Mining in the Large: Preprocessing, Discovery, and Diagnostics

2012-12 Kees van der Sluijs (TUE)

Model Driven Design and Data Integration in Semantic Web Information Systems

2012-13 Suleman Shahid (UvT)

Fun and Face: Exploring non-verbal expressions of emotion during playful interactions

2012-14

Evgeny Knutov(TUE)

Generic Adaptation Framework for Unifying Adaptive Web-based Systems

2012-15 Natalie van der Wal (VU)

Social Agents. Agent-Based Modelling of Integrated Internal and Social Dynamics of

Cognitive and Affective Processes 
2012-16 Fiemke Both (VU)

Helping people by understanding them - Ambient Agents supporting task execution and depression treatment

2012-17 Amal Elgammal (UvT)

Towards a Comprehensive Framework for Business Process Compliance

2012-18 Eltjo Poort (VU)

Improving Solution Architecting Practices

2012-19 Helen Schonenberg (TUE)

What's Next? Operational Support for Business Process Execution

2012-20 Ali Bahramisharif (RUN)

Covert Visual Spatial Attention, a Robust Paradigm for Brain-Computer Interfacing

2012-21 Roberto Cornacchia (TUD)

Querying Sparse Matrices for Information Retrieval

2012-22 Thijs Vis (UvT)

Intelligence, politie en veiligheidsdienst: verenigbare grootheden?

2012-23 Christian Muehl (UT)

Toward Affective Brain-Computer Interfaces: Exploring the Neurophysiology of Affect during Human Media Interaction

2012-24 Laurens van der Werff (UT)

Evaluation of Noisy Transcripts for Spoken Document Retrieval

2012-25 Silja Eckartz (UT)

Managing the Business Case Development in Inter-Organizational IT Projects:

A Methodology and its Application

2012-26 Emile de Maat (UVA)

Making Sense of Legal Text

2012-27 Hayrettin Grkk (UT)

Mind the Sheep! User Experience Evaluation \& Brain-Computer Interface Games

2012-28 Nancy Pascall (UvT)

Engendering Technology Empowering Women

2012-29 Almer Tigelaar (UT)

Peer-to-Peer Information Retrieval

2012-30 Alina Pommeranz (TUD)

Designing Human-Centered Systems for Reflective Decision Making

2012-31 Emily Bagarukayo (RUN)

A Learning by Construction Approach for Higher Order Cognitive Skills Improvement, Building Capacity and Infrastructure

2012-32 Wietske Visser (TUD)

Qualitative multi-criteria preference representation and reasoning

2012-33 Rory Sie (OUN)

Coalitions in Cooperation Networks (COCOON)

2012-34 Pavol Jancura (RUN)

Evolutionary analysis in PPI networks and applications

2012-35 Evert Haasdijk (VU)

Never Too Old To Learn - On-line Evolution of Controllers in Swarmand Modular Robotics

2012-36 Denis Ssebugwawo (RUN)

Analysis and Evaluation of Collaborative Modeling Processes

2012-37 Agnes Nakakawa (RUN)

A Collaboration Process for Enterprise Architecture Creation

2012-38 Selmar Smit (VU)

Parameter Tuning and Scientific Testing in Evolutionary Algorithms

2012-39 Hassan Fatemi (UT)

Risk-aware Design of Value and Coordination Networks 


\section{Samenvatting}

Een samenwerkingsnetwerk is een netwerk dat bestaat uit een verzameling autonome actoren (bijvoorbeeld bedrijven, organisaties en mensen) die samenwerken om gemeenschappelijke of overeenstemmende doelen te bereiken. In een samenwerkingsnetwerk levert iedere onderneming een bijdrage met zijn eigen specifieke producten of diensten om aan de consumentenbehoefte te voldoen. Samenwerkingsnetwerken zijn vaak risicovol, omdat er geen centrale coördinator is. Een samenwerkingsnetwerk dat geleidelijk ontstaat is niet onderworpen aan dezelfde risico's als een samenwerkingsnetwerk dat ontworpen is. In het eerste geval neemt de betrokkenheid van de deelnemers, en daarmee hun risico, langzaam toe, terwijl in het tweede geval de betrokkenheid, en dus het risico, vanaf het begin geldt. Wij ontwikkelen technieken voor het ontwerpen van IT-gebaseerde samenwerkingsnetwerken en voor het valideren van het risico dat ze inhouden. Een deel van de risico's voor een samenwerkingsnetwerk dat is ontworpen zijn:

- het risico van niet-uitvoerbaarheid (geen haalbare coördinatie);

- het risico van fraude of in het algemeen onbetrouwbare partners;

- het risico van niet-winstgevendheid;

- het risico van verlies van vertrouwelijke zakelijke gegevens.

In dit werk richten we ons op de eerste drie soorten risico's.

Het ontwerpen van samenwerkingsnetwerken vereist het modelleren van de samenwerking van bedrijven vanuit verschillende perspectieven, met name de waarde- en coördinatieperspectieven, en het op elkaar afstemmen van deze perspectieven. De behoefte aan deze twee perspectieven komt voort uit het belang van het scheiden van het 'hoe' van de 'wat' zorgen. Het waardeperspectief richt zich op wat wordt aangeboden door wie en aan wie, terwijl het coördinatieperspectief zich richt op hoe datgene wat wordt aangeboden kan worden geöperationaliseerd. Waardemodellering en coördinatiemodellering hebben verschillende doelen en maken gebruik van verschillende concepten. Toch moeten de resulterende 
modellen consistent zijn met elkaar, omdat ze verwijzen naar hetzelfde systeem.

Het model dat de creatie, distributie en consumptie van goederen of diensten van economische waarde in een samenwerkingsnetwerk toont heet waardemodel. Het belangrijkste doel van waardemodellering is om te komen tot een gedeeld begrip van de businesscase en om overeenstemming te bereiken tussen de winst-enverlies verantwoordelijke actoren met betrekking tot de vraag: "Wie biedt wat aan van waarde, aan wie, en verwacht wat van waarde hiervoor terug?" Waardemodellering maakt het voor de actoren ook mogelijk om hun potentiële winstgevendheid te beoordelen en om een inzicht te krijgen in de economische levensvatbaarheid en de duurzaamheid van het samenwerkingsverband.

De studie van de relatie tussen waarde- en coördinatiemodellen is het aanpakken van de uitvoerbaarheid van het gezamenlijke netwerk.

De deelnemende actoren in een samenwerkingsverband worden verondersteld betrouwbaar te handelen. Echter, de veronderstelling dat business actoren betrouwbaar handelen is vaak niet bruikbaar in de praktijk aangezien er ook onbetrouwbare actoren zijn. Elke partner dient toe te treden op basis van het waardemodel en tijdens de samenwerking moeten de actoren er zeker van zijn dat hun partners handelen volgens het waardemodel. Tot slot, nadat we rekening hebben gehouden met complicaties, moeten we de winstgevendheid en duurzaamheid aanpassen aan de nieuwe situatie en dus het risico van verminderde winstgevendheid of zelfs verlies in de samenwerking nemen.

De bijdragen van dit proefschrift zijn:

1. Het introduceren van een stapsgewijze methode om in een multi-perspectief e-collaboratienetwerk een consistent coördinatiemodel te produceren uit een waardemodel, en vice versa.

2. Het analyseren van samenwerkingsnetwerken vanuit een vertrouwensperspectief en het introduceren van een aanpak om de betrouwbaarheid van deelnemers in een samenwerkingsnetwerk te meten.

3. Het beoordelen van de winstgevendheid en duurzaamheid van enkele speciale samenwerkingsnetwerken door middel van concepten en technieken uit de speltheorie en het verfijnen van de initiële winstgevendheidanalyse van samenwerkingsnetwerken door betrouwbaarheid in acht te nemen.

We evalueren onze bevindingen door middel van enkele echte businesscase samenwerkingsverbanden. 